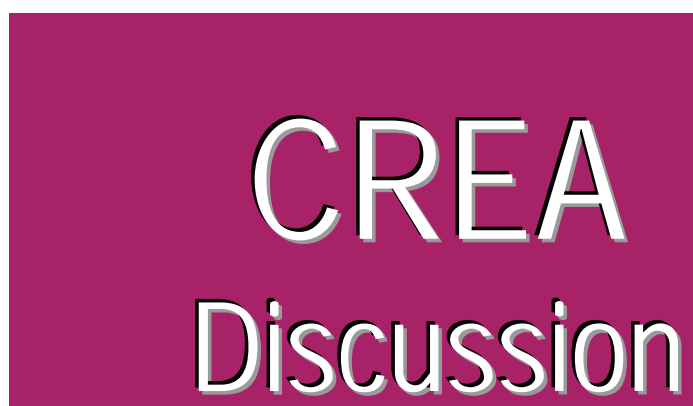
Paper 2017-22 Economics

Center for Research in Economics and Management University of Luxembourg

\title{
Urban Structures with Forward and Backward Linkages
}

available online : http://wwwfr.uni.lu/recherche/fdef/crea/publications2/discussion_papers

Pascal Mossay, Newcastle University, United Kingdom

Pierre M. Picard, CREA, University of Luxembourg Takatoshi Tabuchi, University of Tokyo, Japan

December, 2017

For editorial correspondence, please contact: crea@uni.lu

University of Luxembourg Faculty of Law, Economics and Finance 162A, avenue de la Faïencerie I -1511 I Ixamhnı ırn 


\title{
Urban Structures with Forward and Backward Linkages*
}

\author{
Pascal Mossay ${ }^{\dagger}$ Pierre M. Picard, ${ }^{\ddagger}$ and Takatoshi Tabuchi ${ }^{\S}$
}

December 11, 2017

\begin{abstract}
We study urban structures driven by demand and vertical linkages in the presence of increasing returns to scale. Individuals consume local urban varieties and firms use these varieties to produce a national good. We prove the existence of a spatial equilibrium and obtain an invariance result according to which more intense demand or vertical linkages have the same effect on the urban structure as lower commuting costs. Various urban configurations can emerge exhibiting a monocentric, an integrated, a duocentric, or a partially integrated city structure. We discuss the role of commuting and transport costs, intensities of demand and vertical linkages, and urbanization in affecting these patterns. We show that multiple equilibria may arise in equilibrium involving the monocentric city and up to a couple of duocentric and partially integrated structures.
\end{abstract}

Keywords: Urban spatial structure, demand and vertical linkages, monopolistic competition, land use

J.E.L.: R12, R14, R31

\section{Introduction}

In this paper we study spatial urban structures driven by demand and vertical linkages in the presence of increasing returns to scale. Individuals consume local urban varieties produced by firms under

*We wish to thank Masahisa Fujita, Koichi Fukumura, Jacques Thisse and participants of the Osaka Conference for valuable comments and suggestions.

$\dagger^{\dagger}$ Newcastle University and CORE. Email: pascal.mossay@ncl.ac.uk

$\ddagger$ CREA, University of Luxembourg and CORE, Université catholique de Louvain. Email: pierre.picard@uni.lu

$\S$ Faculty of Economics, University of Tokyo, Hongo 7-3-1, Bunkyo-ku, Tokyo 113-0033, Japan. Email: ttabuchi@e.utokyo.ac.jp. 
monopolistic competition. Firms also use these varieties as intermediate components to produce a homogeneous national good under perfect competition, which is sold within and outside the city.

Our aim is to study how commuting and transport costs, intensities of demand and vertical linkages, and urbanization affect the urban structure. For this purpose, we establish equilibrium conditions in the product and land markets. Our results are the following. First we prove the existence of a spatial equilibrium and obtain an invariance result according to which more intense demand or vertical linkages have the same effect on the urban structure as lower commuting costs. We then study several urban structures and discuss their condition of existence and properties. For the monocentric and integrated cities, we provide explicit conditions that can be represented in terms of the parameters of interest. As for duocentric and partially integrated cities, we derive implicit conditions that determine the borders of business/residential districts. These restrictions on district borders make part of the equilibrium conditions determining the emergence of duocentric and partially integrated patterns.

Our analysis confirms that a monocentric city emerges for very low commuting costs while spatial integration of business and residential districts occurs when goods are shipped at low transport costs within the city. As more intense demand or vertical linkages have the same effect as lower commuting costs, they make the monocentric structure more likely. Furthermore, for intermediate commuting/transport costs and moderate intensities of demand/vertical linkages, many urban structures may emerge and coexist in equilibrium. We show the existence of multiple equilibria involving the monocentric city and up to a couple of duocentric and partially integrated urban structures. Urbanization via an increase in city size induces transitions from the monocentric configuration to full integration possibly passing by duocentric or partially integrated structures depending on the magnitude of the transport cost. When transport costs are high, the economy undergoes discontinuous transitions reflecting larger benefits gained by firms when production locations separate into several business districts.

The present paper extends the existing literature on the endogenous formation of urban areas by uncovering the role of backward and forward linkages on the formation of cities. Whereas Fujita and Ogawa (1982) and Lucas and Rossi-Hansberg (2002) discuss the implications of agglomeration benefits arising from face-to-face communication and exogenous technological spillovers respectively, our paper studies agglomeration forces that are microfounded in the existence of imperfect competition and firms' increasing returns to scale. Accordingly, firms operating in the city have market power and earn a 
positive profit before proceeding to land rent and labor wage payments. The presence of these internal increasing returns makes our analysis more appealing in an urban context. As such, our work fills a gap in the literature. Note that the reduced form of our model encompasses the model structure proposed by Fujita and Ogawa (1982). Namely, in the absence of demand linkages and profit in the production of urban varieties, the reduced form of our model is reminiscent to their seminal work. This suggests that vertical linkages have similar agglomeration effects on the urban structure as those generated by face-to-face communication and technological spillovers. We therefore confirm the generality of the latter approaches. As in Lucas and Rossi-Hansberg (2002), our paper provides an existence proof of spatial equilibrium. However, ours is based on Carlier and Ekeland (2007) and applies to Fujita and Ogawa (1982). Regarding the analysis and computation of multiple equilibria, our approach contrasts with methods relying on fixed point algorithms. We propose an innovative way of determining urban structures, which consists in determining the borders of potential business/residential districts first, and then in checking whether each structure candidate actually constitutes a spatial equilibrium or not.

The paper is structured as follows. Section 2 presents the model and discusses the product and land markets equilibrium. Section 3 establishes the existence of a spatial equilibrium and the invariance result between demand/vertical linkages and commuting costs. Section 4 studies various spatial equilibria exhibiting a monocentric, an integrated, a duocentric, or a partially integrated city structure. Section 5 presents numerical computations and results regarding the structure of these urban patterns. Section 6 concludes.

\section{The model}

We consider a linear city hosting a continuum of $N$ individuals and $N$ firms along the interval $\mathcal{B} \equiv[-b, b] \subset \mathbb{R}$ with unit width. The location supports of individuals and firms are denoted respectively by $\mathcal{X}$ and $\mathcal{Y}$, with $\mathcal{X} \cup \mathcal{Y}=\mathcal{B}$. Individuals occupy a unit of residential land, commute to their workplace, and consume differentiated varieties supplied in the local urban markets as well as an homogeneous good supplied in the national (i.e. intercity) market. In contrast to the national good, trade of local varieties incurs iceberg transport costs. These varieties are also used as components (inputs) to produce the national good. For these two production activities, each firm hires a worker 
and uses $s$ units of land in the production process.

Using the functional form proposed by Pflüger (2004), an individual residing at $x \in \mathcal{X}$ and working at $z \in \mathcal{Y}$ is endowed with the utility function

$$
U(x, z)=\alpha \ln \left[\int_{\mathcal{Y}} c(z, y)^{\frac{\sigma-1}{\sigma}} \mu(y) \mathrm{d} y\right]^{\frac{\sigma}{\sigma-1}}+c_{0}(x)
$$

where $c(z, y)$ is her consumption at workplace location $z$ of a variety produced by a firm located at $y \in \mathcal{Y}, c_{0}(x)$ her consumption of the national good at residence location $x, \mu(y)$ the density of firms at $y \in \mathcal{Y}, \sigma>1$ the elasticity of substitution between local varieties, and $\alpha>0$ the expenditure intensity on local varieties. Her budget constraint writes as

$$
\int_{\mathcal{Y}} p(y) c(z, y) e^{\tau|z-y|} \mu(y) \mathrm{d} y+p_{0} c_{0}(x)+t|x-z|+R(x) \leq w(z)+\bar{c}_{0}
$$

where $p(y)$ is the mill price of a local variety produced at $y, \tau$ the iceberg cost, $c(z, y) e^{\tau|z-y|}$ the quantity of a variety to be purchased at the mill so that consumption is $c(z, y), p_{0}$ the price of the national good, $t$ the commuting cost per unit of distance between residence and work places, $R(x)$ the land rent at the worker's residence, $w(z)$ her wage at the workplace, and finally $\bar{c}_{0}$ some initial endowment of the national good.

On the production side, each firm hires a unit mass of workers, each of them supplying a unit of labor. Each firm simultaneously produces the national good and a single component variety. To highlight the effect of vertical linkages, we assume that the national good is made of the component varieties available in the urban markets. For instance, think of a city exporting wood and metal furniture: woodcraft makers could be exporting wood furniture with steel ornaments made by local steelmakers while steelmakers would be exporting steel furniture with wood ornaments made by local woodcraft subcontractors. Similarly, the city of London hosts law firms that use other law firms' and banks' expertise to provide law services in the national market while banking firms use other banking and law firms' competencies to provide national banking solutions. Therefore, the productions of the national good and local components are integrated within the same plant or office. This is because firm's expertise or supervision is too costly to manage over different plants. Also additional discussion on outsourcing would bring the model out of focus. Finally, the national good is assumed to be homogeneous across firms (and cities) and firms are price takers in this market. ${ }^{1}$ In contrast, each firm

\footnotetext{
${ }^{1}$ Product differentiation and imperfect competition in the national market would not add much to our urban structure analysis.
} 
is a price setter in the market of its own urban variety.

More specifically, production takes place as follows. On the one hand, the national good is made of local inputs with the output of a firm located at $y \in \mathcal{Y}$ given by the production function

$$
q(y)=\beta \ln \left[\int_{\mathcal{Y}} i(y, z)^{\frac{\sigma-1}{\sigma}} \mu(z) \mathrm{d} z\right]^{\frac{\sigma}{\sigma-1}}
$$

where $i(y, z)$ is firm $y$ 's local component use of a variety produced by a firm located at $z \in \mathcal{Y}$ and $\beta>0$ the intensity of vertical linkages. Because of iceberg transport costs, firm $y$ purchases $i(y, z) e^{\tau|x-y|}$ units of a variety produced in $z$ and sold at mill price $p(z)$. On the other hand, the firm produces its specific component using the national good as input. Every component requires $\gamma$ units of the national good. Therefore, firm $y$ 's profit is given by

$$
\pi(y)=\underbrace{p_{0} q(y)-\int_{\mathcal{Y}} p(z) i(y, z) e^{\tau|y-z|} \mu(z) \mathrm{d} z}_{\text {national product market }}+\underbrace{[p(y)-\gamma] \int_{\mathcal{Y}}[c(z, y)+i(z, y)] \mu(z) \mathrm{d} z}_{\text {urban product market }}-w(y)-s R(y)
$$

In this profit expression, the first two terms represent the operating profit in the national market (i.e. revenue minus the cost of inputs), the third term the operating profit in the local component market (i.e. the component markup times the demand from consumers and firms), and the two last terms the labor and land costs. The national good is chosen as the numeraire so that its price can be normalized to $p_{0}=1$.

We now turn to the product market equilibrium where product markets clear.

For this purpose, we consider the locations of firms and individuals as fixed. Assuming $c_{0}(x)>0$, the demand for local varieties by an individual residing at $y$ and working at $z$ is given by

$$
c(z, y)=\alpha \frac{\left[p(y) e^{\tau|z-y|}\right]^{-\sigma}}{P(z)^{1-\sigma}}
$$

where the local price index is given by $P(z) \equiv\left[\int_{\mathcal{Y}} p(y)^{1-\sigma} e^{\tau(1-\sigma)|z-y|} \mu(y) \mathrm{d} y\right]^{\frac{1}{1-\sigma}}$. Her spending on local varieties is equal to $\alpha p_{0}$ and her demand for the national good is given by $c_{0}(x)=[w(z)-t|x-z|$ $\left.-R(x)+\left(\bar{c}_{0}-\alpha\right)\right]$.

On the other hand, a firm located at $y$ simultaneous chooses its input mix $i(y, \cdot)$ and its own component price $p(y)$ that maximize its profit subject to relation (1). Since the firm has a zero mass, it is too small to influence the other firms' local component prices and consumers' demand. Because the profit in the national market has the same functional form as the individual utility, the optimal input 
mix is equal to $i(y, z)=(\beta / \alpha) c(y, z)$ where $c(y, z)$ is given by expression (3). The firm's spending on local varieties is equal to $\beta$. The operating profit on the national market can therefore be written as $\beta[\ln (\beta / P(y))-1]$ so that a firm supplies the national market as long as $\beta>P(y)$. Also, because the total demand for a local variety produced in $y, \int_{\mathcal{Y}}[c(y, z)+i(y, z)] \mathrm{d} z$, has a constant price elasticity $\sigma$, the firm sets its component price to $p(y)=\gamma \sigma /(\sigma-1)>\gamma$, which leads to a positive operating profit in the local component market that turns out to be the same across firms.

The above demand functions and profit-maximizing prices allow us to derive the utility of households and the profit of firms in the product market. First, the price index becomes

$$
P(z)=\gamma \frac{\sigma}{\sigma-1} T(z)
$$

where

$$
T(z) \equiv\left[\int_{\mathcal{Y}} e^{(1-\sigma) \tau|z-y|} \mu(y) \mathrm{d} y\right]^{\frac{1}{1-\sigma}}
$$

is an access measure of location $z$ corresponding to the aggregate delivery cost from all locations on $\mathcal{Y}$ to location $z$, which increases with the transport cost $\tau$ and the dispersion of support $\mathcal{Y}$.

Consumers' and firms' demands for local varieties are given respectively by

$$
\frac{c(z, y)}{\alpha}=\frac{i(z, y)}{\beta}=\frac{\sigma-1}{\gamma \sigma} \frac{e^{-\sigma \tau|z-y|}}{T^{1-\sigma}(z)}
$$

As the demand for the national good is $c_{0}(x)=w(z)-t|x-z|-R(x)+\left(\bar{c}_{0}-\alpha\right)$, the indirect utility writes as

$$
U(x, z)=\alpha\left[\ln \frac{\alpha \rho}{\gamma}-\ln T(z)\right]+\left[w(z)+\bar{c}_{0}-\alpha-t|x-z|-R(x)\right]
$$

where $\rho \equiv 1-1 / \sigma$ and $\mathrm{e}$ is the Euler number. In this expression, the term in the first squared bracket represents the net consumer surplus from local urban varieties while the term in the second squared bracket reflects the net consumer surplus from the national good. The latter is indeed equal to the income left after paying for local urban varieties, commuting cost and land rent. The firm's output in the national market is given by $q^{*}(y)=\beta \ln (\beta \rho / \gamma)-\beta \ln T(y)$.

Using the profit-maximizing price $p(y)=\gamma \sigma /(\sigma-1)$, the gross operating profit from the sales of a local variety to consumers and firms can be written as

$$
[p(y)-\gamma] \int_{\mathcal{Y}}[c(z, y)+i(z, y)] \mu(z) \mathrm{d} z=(\alpha+\beta) G(y)
$$

where profit $G$ is defined by

$$
G(y) \equiv \frac{1}{\sigma} \int_{\mathcal{Y}} \frac{\mu(z)}{T^{1-\sigma}(z)} e^{-\sigma \tau|z-y|} \mathrm{d} z
$$


The expression (2) of the firm's profit at location $y$ can then be written as

$$
\pi(y)=\beta\left[\ln \frac{\beta \rho}{\gamma \mathrm{e}}-\ln T(y)\right]+(\alpha+\beta) G(y)-w(y)-s R(y)
$$

The term in the squared bracket is the profit derived in the national market and the second term that derived in the market of the local variety. The firm has an incentive to produce the national good if the former profit is positive, i.e. $\ln \frac{\beta \rho}{\gamma \mathrm{e}} \geq \ln T(y)$. This holds if $\gamma$ is sufficiently small, which we assume from now on. ${ }^{2}$ The third term $(\alpha+\beta) G(y)$ aggregating the operating profits from the sales of its variety to consumers and firms, increase with the expenditures intensities on local varieties of consumers $(\alpha)$ and firms $(\beta)$. Note that this operating profit clearly depends on the firm's location $y$.

We now turn to the land market equilibrium still assuming the distributions of consumers and firms as given. We assume that land is used either as consumption or firms' input so that the opportunity cost of land is nil. In competitive land markets, landowners allocate their plots of land to the highest bidders (i.e. resident-workers or firms). By using (6), the bit rent of an individual residing at location $x$ and working at $z=z(x)$ is given by

$$
\Psi_{r}(x)=\alpha\left[\ln \frac{\alpha \rho}{\gamma \mathrm{e}}-\ln T(z)\right]+\left[w(z)-t|x-z|+\bar{c}_{0}\right]-U^{*}
$$

where $U^{*}$ denotes the utility she can obtain outside the city. Due to free entry and relation (8), as firm at location $y$ can bid for land up to a level which makes its profit $\pi(y)$ equal to zero, its bid rent is given by

$$
\Psi_{b}(y)=\frac{1}{s}\left\{\beta\left[\ln \frac{\beta \rho}{\gamma \mathrm{e}}-\ln T(y)\right]+(\alpha+\beta) G(y)-w(y)\right\}
$$

As total land consumption $(1+s) N$ is equal to the total available land $2 b$, the city edge is given by $b=(1+s) N / 2$.

\section{Spatial equilibrium}

In this section, we define and study the spatial equilibrium of our economy where both consumers and firms exploit spatial arbitrage opportunities. For this purpose, we rewrite the worker's and the firm's bid rents as follows. The worker's bid rent in location $x$ corresponds to the maximum bid a worker

\footnotetext{
${ }^{2}$ That is, if $\gamma \leq \beta(1-1 / \sigma) /\left(\operatorname{emax}_{\mathrm{y} \in \mathcal{Y}} T(y)\right)$. Given that $\max _{\mathrm{y} \in \mathcal{Y}} T(y)>e^{2 b \tau}$, a sufficient condition is $\gamma<\gamma^{\max } \equiv$ $\beta(1-1 / \sigma) \mathrm{e}^{-2 b \tau-1}$.
} 
can make on a parcel of land in $x$ while receiving a wage from a firm located in $y \in \mathcal{Y}$ willing to hire her under free entry. Using (9) and (8), we get

$$
\begin{aligned}
\Psi_{r}(x)=\max _{y \in \mathcal{Y}, w(y) \in \mathbb{R}^{+}}\left[\alpha \ln \frac{\alpha \rho}{\gamma \mathrm{e}}-\alpha \ln T(y)+w(y)-t|x-y|+\bar{c}_{0}-U^{*}\right] \\
\text { s.t. } \pi(y)=\beta \ln \frac{\beta \rho}{\gamma \mathrm{e}}-\beta \ln T(y)+(\alpha+\beta) G(y)-w(y)-s R(y) \geq 0
\end{aligned}
$$

Similarly, the firm's bid rent corresponds to the maximum bid that it can make on a parcel of land in $y$ while the wage it pays to a worker located in $x \in \mathcal{X}$ induces her to supply her labor to the firm given her outside utility $U^{*}$. Using (10) and (6)

$$
\begin{array}{r}
\Psi_{b}(y)=\max _{x \in \mathcal{X}, w(y) \in \mathbb{R}^{+}} \frac{1}{s}\left[\beta \ln \frac{\beta \rho}{\gamma \mathrm{e}}-\beta \ln T(y)+(\alpha+\beta) G(y)-w(y)\right] \\
\text { s.t. } U(x, y)=\alpha \ln \frac{\alpha \rho}{\gamma \mathrm{e}}-\alpha \ln T(x)+w(y)-t|x-y|-R(x)+\bar{c}_{0} \geq U^{*}
\end{array}
$$

We now make the connection with Fujita and Ogawa (1982). In their seminal paper, urban structures endogenously emerge from agglomeration effects generated by face-to-face communication between firms' managers and/or employees. This face-to-face communication generates a production surplus that depends negatively on the logarithm of the average distance between firms. One could then interpret the parameter expression $(\sigma-1) \tau$ in $\ln T(y)$ as Fujita and Ogawa's parameter for faceto-face communication. This means that from a reduced form point of view, vertical linkages have the same role as face-to-face communication in Fujita and Ogawa. However, our vertical linkages also stem from the production of urban varieties under imperfect competition, which generates a profit $G(y)$ that is absent in the face-to-face communication model. To sum up, the reduced form of our model encompasses the structure of Fujita and Ogawa in the absence of demand linkages and profit from production of urban varieties (that is, $\alpha=0$ and $G(y)=0$ ).

The above problems can be solved for the wage so that

$$
\begin{aligned}
& \Psi_{r}(x)=\max _{y \in \mathcal{Y}}\left[\alpha \ln \frac{\alpha \rho}{\gamma \mathrm{e}}+\beta \ln \frac{\beta \rho}{\gamma \mathrm{e}}+(\alpha+\beta) S(y)-t|x-y|+\bar{c}_{0}-U^{*}-s R(y)\right] \\
& \Psi_{b}(y)=\frac{1}{s} \max _{x \in \mathcal{X}}\left[\alpha \ln \frac{\alpha \rho}{\gamma \mathrm{e}}+\beta \ln \frac{\beta \rho}{\gamma \mathrm{e}}+(\alpha+\beta) S(y)-t|x-y|+\bar{c}_{0}-U^{*}-R(x)\right]
\end{aligned}
$$

where $S(y) \equiv G(y)-\ln T(y)$ is the economic surplus in location $y$.

We can now define a competitive spatial equilibrium.

Definition 1 A competitive spatial equilibrium is defined by the residents' and firms' density functions $\lambda: \mathcal{B} \rightarrow[0,1]$ and $\mu: \mathcal{B} \rightarrow[0,1 / s]$, a land rent $R: \mathcal{B} \rightarrow \mathbb{R}^{+}$, residents' and firms' bid rent functions 
$\Psi_{r}$, and $\Psi_{b}: \mathcal{B} \rightarrow \mathbb{R}^{+}$and a utility level $U^{*} \in \mathbb{R}$ that satisfy (i) mass conservation $\int_{\mathcal{B}} \lambda=N$, (ii) land market clearing $\mu(z)=(1-\lambda(z)) / s$ for all $z \in \mathcal{B}$, (iii) land allocation to highest bidder for all $z \in \mathcal{B}$

$$
\lambda \in\left\{\begin{array}{lll}
\{1\} & \text { if } & \Psi_{r}>\Psi_{b} \\
{[0,1]} & \text { if } & \Psi_{r}=\Psi_{b} \\
\{0\} & \text { if } & \Psi_{r}<\Psi_{b}
\end{array}\right.
$$

and $R=\max \left\{\Psi_{r}, \Psi_{b}, 0\right\}$, and (iv) zero land rent $R$ at city borders $z= \pm b$.

\subsection{Economic gains and invariance}

The urban economic activity generates gains for workers and landlords, firms making zero profit due to free entry. It is instructive to examine those gains and disentangle the agglomeration force stemming from demand and vertical linkages from the dispersion force due to transportation, and in particular, commuting costs.

Consider a worker residing at $x$ and its firm producing at $y$ at the competitive land market equilibrium. Land rent should correspond to their respective bid rents: $\Psi_{r}(x)=R(x)$ and $\Psi_{r}(y)=R(y)$. Therefore, from (13) or (14), the total gains from their urban economic interaction are

$$
U^{*}+R(x)+s R(y)=\alpha \ln \frac{\alpha \rho}{\gamma \mathrm{e}}+\beta \ln \frac{\beta \rho}{\gamma \mathrm{e}}+(\alpha+\beta) S(y)-t|x-y|+\bar{c}_{0}
$$

While the left hand side expresses workers' utility and landlords' rents, the right hand side reflects the consumer surplus from local varieties and the producer surplus from both consumers and firms and from the national market (through terms in $\alpha$ and $\beta$ ). The surpluses are explicitly diminished by commuting costs.

Observe that the spatial structure $(\lambda, \mu)$ has an impact only on the terms $(\alpha+\beta) S(y)-t|x-y|$. This means that it is possible to maintain the same equilibrium spatial structure $\left(\lambda^{*}, \mu^{*}\right)$ by multiplying both parameters $(\alpha+\beta)$ and $t$ by the same factor $k>0$. To maintain the above equality for all $x$ and $y$, equilibrium rents need to be multiplied by this factor $k$ and the equilibrium utility $U^{*}$ increased to the level $\left(k \alpha \ln \frac{\alpha k \rho}{\gamma \mathrm{e}}+k \beta \ln \frac{k \beta \rho}{\gamma \mathrm{e}}\right)$. This heuristic argument is confirmed below in a formal way.

Suppose that a spatial equilibrium exists and denote the mass of commuters moving to the right and crossing location $x$ by $n(x)=\int_{-b}^{x}[\lambda(z)-\mu(z)] \mathrm{d} x$. In Appendix A, we show that the spatial 
equilibrium $(\lambda, \mu)$ satisfies the identity

$$
\lambda(z)-\mu(z) \in\left\{\begin{array}{lll}
\{1\} & \text { if } & \Psi_{b}(z)-\Psi_{r}(z)<0 \\
{\left[-\frac{1}{s}, 1\right]} & \text { if } & \Psi_{b}(z)-\Psi_{r}(z)=0 \\
\left\{-\frac{1}{s}\right\} & \text { if } & \Psi_{b}(z)-\Psi_{r}(z)>0
\end{array}\right.
$$

where

$$
\Psi_{b}(z)-\Psi_{r}(z)=\int_{\widehat{z}}^{z}\left[\frac{1}{s}(\alpha+\beta) \frac{\mathrm{d} S}{\mathrm{~d} x}-\frac{1+s}{s} t \operatorname{sign}(n(x))\right] \mathrm{d} x,
$$

and $\widehat{z} \in \mathcal{B}$ is a solution to $\lambda(\widehat{z})-\mu(\widehat{z})=0$ and designates a location where commuting stops or changes direction. There always exists is at least one such location. Bid rents equate in those locations $\widehat{z}$ since workers and firms settle either on the same land plot or on two infinitely close residential and industrial land plot. The first term in the integral yields the economic surplus $S^{*}(x)$ (up to a constant) generated at location $x$. The second term reflects the travel cost of commuters crossing location $x$. Of course, we can use the relationship $\lambda=1-s \mu$ to eliminate a density profile either $\lambda$ or $\mu$.

The above identity tells that workers and firms settle in the locations where they respectively place the highest bid rents. When they offer the same bid, any share of firms and residents is possible. In this competitive allocation, the spatial equilibrium depends on the sign of the bid rent differentials but not on their amplitudes. Hence multiplying both the parameters $(\alpha+\beta)$ and $t$ by any scalar $k>0$ has no effect on the sign of the bid differentials and therefore is compatible with the spatial equilibrium structure. This yields the following main result:

Proposition 1 Cities with a same parameter ratio $(\alpha+\beta) / t$ have an identical spatial structure.

The ratio shows that stronger demand or vertical linkages and higher commuting costs have opposite effects on the urban structure. The economic surplus generated by any pair of worker and a firm is balanced with the commuting cost between the worker's residence and her firm. A proportional increase in the intensity of demand or vertical linkages and in commuting cost does not change this

spatial balance though each effect becomes more intense. As a result, stronger linkages have the same impact as lower commuting costs.

\section{$3.2 \quad$ Existence}

Relationships (16) and (17) define a fixed point problem for the firms' spatial distribution. Indeed, the firms' density $\mu$ determines the values of the surplus $S$ which in turn determines $\mu$. In Appendix 
B, we show the existence of a fixed point using Shauder's theorem.

Proposition 2 A spatial equilibrium exists.

The proof of spatial equilibrium existence relies on the determination of the stock of commuters flowing over each urban location, $n(x)$. This is referred as the 'stock of unhoused workers' by Lucas and Rossi-Hansberg (2002). As shown in (16), the main difficulty in establishing the existence of spatial equilibrium lies in the discontinuity of the land allocation process across firms and workers. As in Carlier and Ekeland (2007), we first show the existence of a fixed point for smoother land allocation processes by replacing (16) by an equation where the land allocation decision across firms and workers is a continuous function of their bid rent differential $\Psi_{b}-\Psi_{r}$. In economic terms, this may be interpreted by the presence of exogenous uncertainty in landlords' choices. We then prove the convergence of those fixed points to that defined by (16) and (17) for less and less smooth allocation processes. Using the same interpretation, the fixed point takes place at the limit of zero uncertainty in the land allocation process. It must be noted that the above existence result applies to Fujita and Ogawa's (1982) model which lacks a general existence proof and displays parameter values for which no equilibrium could be constructed. As mentioned earlier, their equilibrium conditions are structurally the same as ours if one sets $\alpha$ and $G$ to zero and interprets $(\sigma-1) \tau$ as a parameter for face-to-face communication.

\section{Urban structures}

Our existence result in Section 3 says nothing about uniqueness of equilibrium. In this section, we present and discuss a variety of urban structures and determine their condition of existence.

\subsection{Monocentric city}

We consider the business district $\mathcal{Y}=\left[-b_{1}, b_{1}\right]$ surrounded by the residential districts $\mathcal{X}=\left[-b,-b_{1}\right) \cup$ $\left(b_{1}, b\right]$, see Figure 1 (a). The densities of firms and households are $\mu(y)=1 / s$ and 1 respectively. The business district edge is given by $b_{1}=s N / 2$. In the monocentric city, workers commute from the residential district to the business district. 
The monocentric equilibrium conditions are as follows: $(i)$ firms outbid households in the business district $\left(\Psi_{b}(y) \geq \Psi_{r}(y)\right.$ for all $\left.y \in \mathcal{Y}\right),(i i)$ households outbid firms in the residential district $\left(\Psi_{r}(x) \geq\right.$ $\Psi_{b}(x)$ for all $\left.x \in \mathcal{X}\right) ;(i i i)$ the residential and the business bid rents equalize at the business district border $\left(\Psi_{b}\left(b_{1}\right)=\Psi_{r}\left(b_{1}\right)\right) ;(i v)$ the zero opportunity cost of land at the city border $b$ requiring $\Psi_{r}(b)=0$.

As of expressions (4) and (7), we define the surplus at location $z$ as $S_{M}(z) \equiv G_{M}(z)-\ln T_{M}(z){ }^{3}$ where the access measure $T_{M}$ and the profit $G_{M}$ are given by

$$
\begin{aligned}
T_{M}^{1-\sigma}(z) & =\frac{1}{s} \int_{-b_{1}}^{b_{1}} e^{(1-\sigma) \tau|y-z|} \mathrm{d} y \\
G_{M}(z) & =\frac{1}{\sigma s} \int_{-b_{1}}^{b_{1}} \frac{e^{-\sigma \tau|y-z|}}{T_{M}^{1-\sigma}(y)} \mathrm{d} y
\end{aligned}
$$

Proposition 3 The monocentric configuration is a spatial equilibrium if and only if

$$
t \leq t_{M}=\min \left(\widehat{t}_{M}, \bar{t}_{M}\right)
$$

where

$$
\bar{t}_{M}=\frac{\alpha+\beta}{1+s} \frac{S_{M}(0)-S_{M}(s N / 2)}{s N / 2} \quad \text { and } \widehat{t}_{M} \equiv \frac{\alpha+\beta}{1+s} \frac{S_{M}(s N / 2)-S_{M}((1+s) N / 2)}{N / 2}
$$

When $s \rightarrow 0, \bar{t}_{M}=(\alpha+\beta) \tau N<\widehat{t}_{M}$.

Proof. Given the symmetry of the city, our analysis focuses on the right side of the city $[0, b]$.

Under linear commuting costs, workers can arbitrage between the different workplaces. As a result, a firm located at $y$ must offer individuals a wage $w(y)$ that is as attractive as that available in other work locations. Denoting $U_{0}$ by $\alpha\left[\ln (\alpha \rho / \gamma \mathrm{e})-\ln T_{M}(0)\right]+w(0)+\bar{c}_{0}$, we can write the following wage arbitrage condition for any $0 \leq y<x$ :

$$
\alpha\left[\ln \frac{\alpha \rho}{\gamma \mathrm{e}}-\ln T_{M}(y)\right]+w(y)+t y+\bar{c}_{0}=U_{0}
$$

so that the equilibrium wage $w(y)$ satisfies

$$
w(y)=U_{0}-\alpha\left[\ln \frac{\alpha \rho}{\gamma \mathrm{e}}-\ln T_{M}(y)\right]-t y-\bar{c}_{0}
$$

By using this wage expression, the household's bid rent (9) can then be written as

$$
\Psi_{r}(x)=\left(U_{0}-U^{*}\right)-t x
$$

\footnotetext{
${ }^{3}$ The surplus $S_{M}(z)$ corresponds to the profit in the market of a local variety accounting for the cost of accessing all components in order to produce the national good.
} 
Similarly, the firm's bid rent (10) writes as

$$
\Psi_{b}(y)=\frac{1}{s}\left[\left(\alpha \ln \frac{\alpha \rho}{\gamma \mathrm{e}}+\beta \ln \frac{\beta \rho}{\gamma \mathrm{e}}-U_{0}+\bar{c}_{0}\right)+(\alpha+\beta) S_{M}(y)+t y\right]
$$

At the land market equilibrium, landlords reap the surplus derived by firms and households.

As the district border is given by $b_{1}=s N / 2$, we need to impose conditions $(i),(i i),(i i i)$, and (iv) on the bid rent functions (20) and (21) of residents and firms. It is shown in Appendix C that conditions $(i)$ and (ii) reduce to $\left(i^{\prime}\right) \Psi_{b}(0) \geq \Psi_{r}(0)$ and $\left(i i^{\prime}\right) \Psi_{r}(b) \geq \Psi_{b}(b)$ respectively.

Condition $\left(i^{\prime}\right) \Psi_{b}(0) \geq \Psi_{r}(0)$ can be rewritten as

$$
\frac{1}{s}\left(\alpha \ln \frac{\alpha \rho}{\gamma \mathrm{e}}+\beta \ln \frac{\beta \rho}{\gamma \mathrm{e}}+\bar{c}_{0}-U_{0}\right)+\frac{1}{s}(\alpha+\beta) S_{M}(0) \geq\left(U_{0}-U^{*}\right)
$$

The equilibrium conditions (iii) and $(i v)\left(\Psi_{b}\left(b_{1}\right)=\Psi_{r}\left(b_{1}\right)\right.$ and $\left.\Psi_{r}(b)=0\right)$ determine $U_{0}$ and $U^{*}$. In particular, we have

$$
U_{0}=\left(\alpha \ln \frac{\alpha \rho}{\gamma \mathrm{e}}+\beta \ln \frac{\beta \rho}{\gamma \mathrm{e}}+\bar{c}_{0}\right)+(\alpha+\beta) S_{M}\left(b_{1}\right)+(1+s) t b_{1}-s t b
$$

By plugging the above expression of $U_{0}$ into (22) and using $b_{1}=s N / 2$, we get

$$
t \leq \bar{t}_{M} \equiv \frac{2(\alpha+\beta)}{(1+s) s N}\left[S_{M}(0)-S_{M}\left(b_{1}\right)\right]
$$

When $s \rightarrow 0$, we have

$$
\lim _{s \rightarrow 0} \bar{t}_{M}=-(\alpha+\beta) \lim _{s \rightarrow 0} \frac{S_{M}(s N / 2)-S_{M}(0)}{s N / 2}=\frac{(\alpha+\beta) \tau}{N}
$$

Furthermore, we also have

$$
\begin{aligned}
\Psi_{b}(b) & =\frac{1}{s}\left[(\alpha+\beta) S_{M}(b)+t b+\left(\alpha \ln \frac{\alpha \rho}{\gamma \mathrm{e}}+\beta \ln \frac{\beta \rho}{\gamma \mathrm{e}}-U_{0}+\bar{c}_{0}\right)\right] \\
& =\frac{1}{s}\left[(\alpha+\beta)\left[S_{M}(b)-S_{M}\left(b_{1}\right)\right]+(1+s) N \frac{t}{2}\right]
\end{aligned}
$$

so that the condition $\left(i i^{\prime}\right), \Psi_{b}(b) \leq \Psi_{r}(b)=0$, can now be rewritten as

$$
t \leq \widehat{t}_{M} \equiv \frac{2(\alpha+\beta)}{(1+s) N}\left[S_{M}\left(b_{1}\right)-S_{M}(b)\right]
$$

Gathering expressions (23) and (24) leads to $t \leq \min \left(\widehat{t}_{M}, \bar{t}_{M}\right)$.

When $s \rightarrow 0$, we get

$$
\begin{aligned}
\lim _{s \rightarrow 0}\left[\ln T_{M}(s N / 2)-\ln T_{M}((1+s) N / 2)\right] & =-\tau N / 2 \\
\lim _{s \rightarrow 0}\left[G_{M}(s N / 2)-G_{M}((1+s) N / 2)\right] & =\frac{1-e^{-\sigma \tau N / 2}}{\sigma}
\end{aligned}
$$


Therefore

$$
\begin{aligned}
\lim _{s \rightarrow 0} \widehat{t}_{M} & \equiv 2(\alpha+\beta) \lim _{s \rightarrow 0}\left[S_{M}(s N / 2)-S_{M}((1+s) N / 2)\right] \\
& =2(\alpha+\beta) \lim _{s \rightarrow 0}\left[G_{M}(s N / 2)-G_{M}((1+s) N / 2)-\ln T_{M}(s / 2)+\ln T_{M}((1+s) N / 2)\right] \\
& =(\alpha+\beta)\left(2 \frac{1-e^{-\sigma \tau N / 2}}{\sigma}+\tau N\right)>(\alpha+\beta) \tau N=\lim _{s \rightarrow 0} \bar{t}_{M}
\end{aligned}
$$

so that $\lim _{s \rightarrow 0} \widehat{t}_{M}<\lim _{s \rightarrow 0} \bar{t}_{M}$.

Proposition 3 says that lower commuting costs favor the monocentric configuration as they allow residents to commute longer distances to the business district. Larger final demand or vertical linkages also make the monocentric configuration more likely as they increase the operating profit in the market of local varieties due to increasing returns to scale, thus allowing firms in the business district to pay higher wages and making people more willing to commute.

Figure 2 illustrates the shape of bid rents in a monocentric city. The firms' bid rent curve, represented in red, is above (resp. below) the residents' bid rent curve (in blue) around the city center (resp. the city edge). Bid rents are equal to the zero opportunity cost of land at the city border and intersect at the location $y= \pm 0.5$, which separates business and residential districts. Workers' bid rents are linear in the distance to the center as longer commutes are compensated by lower rents. As firms benefit from agglomeration economies, their bid rent is endogenous and depends on the location of other firms. Typically it is non-linear in the distance to the city center. A priori, it is not obvious to guarantee a single intersection between bid rents. Nevertheless, according to the above Proposition, it turns out that only the bid rent properties at the city center and border matter. While the condition $t \leq \widehat{t}_{M}$ guarantees that firms do not overbid workers at the city border $\left(\Psi_{b}(b) \leq \Psi_{r}(b)\right)$, the condition $t \leq \bar{t}_{M}$ guarantees that they are not overbid by residents at the city center $\left(\Psi_{b}(0) \geq \Psi_{r}(0)\right)$.

The last statement in Proposition 3 implies that, even if the manufacturing sector makes no use of land $(s \rightarrow 0)$, the monocentric city structure may not be a spatial equilibrium. This qualifies the standard discussion based on the monocentric city model where firms are assumed to be located in a city business center using no space. This paper highlights the role of the trade-off between workers' commuting cost and goods' transport costs.

Finally, note that, when transport costs vanish $(\tau \rightarrow 0)$, the economic surplus $S_{M}(z)$ becomes location independent and the above thresholds become $\bar{t}_{M}=\widehat{t}_{M}=0$. Then, the monocentric city is never a spatial equilibrium for any commuting cost $t>0$. In this case, the demand for final goods 
and intermediate goods demand is unrelated to space. There is no agglomeration force and spatial concentration brings no benefit.

\subsection{Integrated city}

We consider firms and households locating in $\mathcal{X}=\mathcal{Y}=[-b, b]$, see Figure 1 (b). Given the unit demand for land of households and the $s$ unit demand of firms, the density of firms is $\mu(y)=1 /(1+s)$ and the city edge is still at $b=(1+s) N / 2$. The integrated configuration involves no commuting.

The integrated equilibrium conditions are as follows: $(i)$ the business and residential bid rents equalize $\left(\Psi_{r}(z)=\Psi_{b}(z), \forall z \in \mathcal{X}=\mathcal{Y}\right) ;(i i)$ the no-arbitrage relocation implying $\left|\Psi_{r}^{\prime}(z)\right| \leq t ;($ iii $)$ the zero opportunity cost of land at the city border $b$ requiring $\Psi(b)=0$.

As of expressions (4) and (7), we define the surplus at location $z$ as $S_{I}(z) \equiv G_{I}(z)-\ln T_{I}(z)$ with the access measure $T_{I}$ and the profit $G_{I}$ given by

$$
\begin{aligned}
T_{I}^{1-\sigma}(z) & =\frac{1}{1+s} \int_{-b}^{b} e^{(1-\sigma) \tau|z-y|} \mathrm{d} y \\
G_{I}(z) & =\frac{1}{\sigma(1+s)} \int_{-b}^{b} \frac{e^{-\sigma \tau|y-z|}}{T_{I}^{1-\sigma}(y)} \mathrm{d} y
\end{aligned}
$$

Proposition 4 The integrated city is a spatial equilibrium if

$$
t \geq t_{I}=-\frac{\alpha+\beta}{1+s} S_{I}^{\prime}(b)>0
$$

When $s \rightarrow 0, t_{I}=(\alpha+\beta) S_{I}^{\prime}(b)$.

Proof. As before, we focus on $[0, b]$. We need to impose the above conditions $(i)-(i i i)$ on the bid rent functions. The equilibrium condition $(i), \Psi_{r}=\Psi_{b}$, leads to

$$
w(y)=\frac{1}{1+s}(\alpha s-\beta) \ln T_{I}(y)+\frac{\alpha+\beta}{1+s} G_{I}(y)+C
$$

where $C \equiv s /(1+s)\left\{U^{*}-\bar{c}_{0}-\alpha \ln [\alpha \rho /(\gamma \mathrm{e})]+\beta / s \ln [\alpha \rho /(\gamma \mathrm{e})]\right\}$.

From equilibrium condition $(i i i), \Psi(b)=0$, we have

$$
\alpha\left[\ln \frac{\alpha \rho}{\gamma \mathrm{e}}-\ln T_{I}(b)\right]+w(b)+\bar{c}_{0}-U^{*}=0
$$

From relations (27) and (28), the constant $C$ can be determined

$$
C=\frac{s}{1+s}(\alpha+\beta) S_{I}(b)+\beta \ln \frac{\beta \rho}{\gamma \mathrm{e}}
$$


By plugging the wage expression (27) into (10) (or (9)), we get the equilibrium land rent

$$
R(z)=\frac{\alpha+\beta}{1+s}\left[S_{I}(z)-S_{I}(b)\right]
$$

According to the no-arbitrage condition $(i i)$, an urban configuration is an equilibrium if workers have no incentive to commute to any other location, i.e., if $\left|R^{\prime}(z)\right|=\left|(\alpha+\beta) /(1+s) S_{I}^{\prime}(z)\right| \leq t$, for $\forall z \in[0, b]$. In Appendix D, it is shown that $\left|S_{I}^{\prime}(z)\right| \leq\left|S_{I}^{\prime}(b)\right|, \forall z \in[0, b]$, so that the no-arbitrage condition reduces to $\left|R^{\prime}(b)\right|=\left|(\alpha+\beta) /(1+s) S_{I}^{\prime}(b)\right| \leq t$.

Proposition 4 states that higher commuting costs foster the integrated configuration. Intuitively, very large commuting costs entice workers to take residence as close as possible to their workplace. Also, weaker final demand or vertical linkages make the integrated configuration more likely. They indeed decrease the operating profits from the production of local varieties, which reduces workers' wages, and in turn the incentives to commute. Finally, it turns out that $t_{I}$ is an increasing function of $\tau$ (see Appendix D). So, lower trade costs enlarge the domain of economic parameters for which firms integrate with residents. For lower trade costs, input costs depend less on distance between firms and vertical linkages lead to a weaker agglomeration force for the firms. Therefore, firms do not benefit much from clustering with other firms and make land rent bids that can be matched by workers.

Figure 3 illustrates the shape of bid rents in an integrated city. The firms' and the residents' bid rent curves coincide at any location in the city. Bid rents vanish at the city borders due to the zero opportunity cost of land. As workers do not commute, rents do not reflect linear commuting costs but rather non-linear agglomeration economies coming from demand and input-output linkages. In the above Proposition, the equilibrium condition $t \geq t_{I}$ guarantees that each worker has no incentive to accept a job away from her residential location. This property obtains if bid rents from are not too steep. It is ensured as long as demand and input-output linkages are weak enough (low $\alpha+\beta)$.

When the transport cost becomes negligible $(\tau \rightarrow 0)$, the economic surplus $S_{I}(z)$ is location independent so that $S_{I}^{\prime}(z)=0$ and $t_{I}=0$. The integrated city is then a spatial equilibrium for any commuting cost $t>0$. In this case, the demands for final goods and intermediate goods are independent of space and the agglomeration force vanish.

Finally, we prove the following corollary:

Corollary 1 For $s \rightarrow 0$, the monocentric and the integrated urban configurations never coexist. That is, $t_{M}<t_{I}$. 


\section{Proof. See Appendix E.}

When the manufacturing sector makes no use of land, a high commuting cost is compatible only with the integrated structure. This results from the tension between backward and forward linkages and commuting costs. When backward and forward linkages are too weak, firms are unable to post land bids that are high enough to foreclose the workers to reside close to them. The above result forbids the possibility of multiple equilibria, where the monocentric and integrated structures coexist for the same set of parameter values. To the best of our knowledge, this property is not well emphasized in the literature (e.g. Fujita and Ogawa 1982, and followers).

\subsection{Partially integrated city}

Next, we consider a city where workers and firms locate with each other in the central district $\left(-b_{1}, b_{1}\right)$, which is surrounded by business districts $\left(-b_{2},-b_{1}\right) \cup\left(b_{1}, b_{2}\right)$, themselves bordered by residential districts $\left(-b,-b_{2}\right) \cup\left(b_{2}, b\right)$, see Figure 1 (c). The density of firms is $\mu(y)=s /(1+s)$ in the interval $\left(-b_{1}, b_{1}\right)$ and $\mu(y)=1 / s$ in the intervals $\left(-b_{2},-b_{1}\right) \cup\left(b_{1}, b_{2}\right)$, while that of workers is $\lambda(x)=1 /(1+s)$ in the interval $\left(-b_{1}, b_{1}\right)$ and $\lambda(x)=1$ in the intervals $\left(-b,-b_{2}\right) \cup\left(b_{2}, b\right)$, with $0<b_{1}<b_{2}<b$. Because the total mass of firms is $N$, we get

$$
2\left(b_{2}-b_{1}\right) \frac{1}{s}+2 b_{1} \frac{1}{1+s}=N
$$

that is,

$$
b_{2}=\frac{b_{1}}{1+s}+\frac{s N}{2}
$$

The partially integrated equilibrium conditions are as follows: $(i)$ the bid rents of households and firms equalize in the integrated district $\left(\Psi_{r}(z)=\Psi_{b}(z), \forall z \in\left(-b_{1}, b_{1}\right)\right)$; $(i i)$ no-arbitrage relocation in the integrated district implying $\left|\Psi^{\prime}(z)\right| \leq t, \forall z \in\left(-b_{1}, b_{1}\right)$; (iii) firms outbid households in the business districts $\left(\Psi_{b}(y) \geq \Psi_{r}(y) \forall y \in\left[-b_{2},-b_{1}\right] \cup\left[b_{1}, b_{2}\right]\right) ;(i v)$ households outbid firms in residential districts $\left(\Psi_{r}(x) \geq \Psi_{b}(x), \forall x \in\left[-b,-b_{2}\right] \cup\left[b_{2}, b\right]\right)$; and $(v)$ the zero opportunity cost of land at the city border $b$ requiring $\Psi_{r}(b)=0$.

As of expressions (4) and (7), we define $S_{P}(z) \equiv G_{P}(z)-\ln T_{P}(z)$ where the access measure $T_{P}$ 
and the profit $G_{P}$ are given by

$$
\begin{aligned}
T_{P}^{1-\sigma}(z) & =\frac{1}{s}\left(\int_{-b_{2}}^{-b_{1}}+\int_{b_{1}}^{b_{2}}\right) e^{(1-\sigma) \tau|z-y|} \mathrm{d} y+\frac{1}{1+s} \int_{-b_{1}}^{b_{1}} e^{(1-\sigma) \tau|z-y|} \mathrm{d} y \\
G_{P}(z) & =\frac{1}{\sigma s}\left(\int_{-b_{2}}^{-b_{1}}+\int_{b_{1}}^{b_{2}}\right) \frac{e^{-\sigma \tau|z-y|}}{T_{P}^{1-\sigma}(y)} \mathrm{d} y+\frac{1}{\sigma(1+s)} \int_{-b_{1}}^{b_{1}} \frac{e^{-\sigma \tau|z-y|}}{T_{P}^{1-\sigma}(y)} \mathrm{d} y
\end{aligned}
$$

Proposition 5 The partially integrated city is a spatial equilibrium if and only if

$$
\widehat{t}_{P} \leq t \leq t_{P}^{*}
$$

where the thresholds $\widehat{t}_{P}$ and $t_{P}^{*}$ are given by

$$
\widehat{t}_{P} \equiv \frac{\alpha+\beta}{1+s} \max _{z \in\left[0, b_{1}\right)}\left|S_{P}^{\prime}(z)\right| ; t_{P}^{*} \equiv \min \left\{\bar{t}_{P}, \tilde{t}_{P}\right\}
$$

with

$$
\widetilde{t}_{P} \equiv \frac{\alpha+\beta}{1+s} \frac{S_{P}\left(b_{2}\right)-S_{P}(b)}{b-b_{2}} ; \bar{t}_{P} \equiv \min _{y \in\left[b_{1}, b_{2}\right]} \frac{\alpha+\beta}{1+s} \frac{S_{P}(y)-S_{P}\left(b_{2}\right)}{b_{2}-y}
$$

The district borders $\left(b_{1}, b_{2}\right)$ satisfy

$$
t=\frac{\alpha+\beta}{1+s} \frac{S_{P}\left(b_{1}\right)-S_{P}\left(b_{2}\right)}{b_{2}-b_{1}}
$$

where $b_{2}$ is given by (29) and $0<b_{1}<b_{2}<N$.

Proof. As before, we focus on $[0, b]$. In the integrated district of the city, condition $(i)$ writes $R(z)=\Psi_{r}(z)=\Psi_{b}(z)$. From expressions (9) and (10), we get

$$
\begin{aligned}
& R(z)=\frac{\alpha+\beta}{1+s} S_{P}(z)+\frac{1}{1+s}\left(\alpha \ln \frac{\alpha \rho}{\gamma \mathrm{e}}+\beta \ln \frac{\beta \rho}{\gamma \mathrm{e}}+\bar{c}_{0}-U^{*}\right), \quad z \in\left[0, b_{1}\right) \\
& w(z)=\frac{\alpha s-\beta}{1+s} \ln T_{P}(z)+\frac{\alpha+\beta}{1+s} G_{P}(z) \\
& -\frac{s}{1+s}\left(\alpha \ln \frac{\alpha \rho}{\gamma \mathrm{e}}-\frac{\beta}{s} \ln \frac{\beta \rho}{\gamma \mathrm{e}}+\bar{c}_{0}-U^{*}\right), \quad z \in\left[0, b_{1}\right)
\end{aligned}
$$

In the residential district, we have $R(x)=\Psi_{r}(x), x \in\left[b_{2}, b\right]$. However, since the residential bid rent $\Psi_{r}(x)$ given by $(9)$ is independent of the workplace $y$ in business district $\left[b_{1}, b_{2}\right]$, it can be written as

$$
R(x)=\Psi_{r}(x)=A-t x, \quad x \in\left[b_{2}, b\right]
$$

where

$$
A=\alpha\left[\ln \frac{\alpha \rho}{\gamma \mathrm{e}}-\ln T_{P}(y)\right]+w(y)+t y+\bar{c}_{0}-U^{*}, \quad y \in\left[b_{1}, b_{2}\right]
$$


is constant with respect to $y$. Condition $(v)$ says that the land rent is nil at $b$, from which we get $A=t b$. So, from the last two expressions, we can write the equilibrium land rent in the residential district and the wage in the business district as

$$
\begin{aligned}
& R(x)=t(b-x), \quad x \in\left(b_{2}, b\right] \\
& w(y)=t(b-y)-\alpha\left[\ln \frac{\alpha \rho}{\gamma \mathrm{e}}-\ln T_{P}(y)\right]-\bar{c}_{0}+U^{*}, \quad y \in\left[b_{1}, b_{2}\right]
\end{aligned}
$$

Inserting the wage (36) into $R(y)=\Psi_{b}(y)$ as given by relation (10) yields the land rent in the business district

$$
R(y)=\frac{1}{s}\left[(\alpha+\beta) S_{P}(y)-t(b-y)+\alpha \ln \frac{\alpha \rho}{\gamma \mathrm{e}}+\beta \ln \frac{\beta \rho}{\gamma \mathrm{e}}+\bar{c}_{0}-U^{*}\right], \quad y \in\left[b_{1}, b_{2}\right]
$$

As land rents are continuous, $R\left(b_{1}-0\right)=R\left(b_{1}+0\right)$ and $R\left(b_{2}-0\right)=R\left(b_{2}+0\right)$, we get

$$
U^{*}=(\alpha+\beta) S_{P}\left(b_{2}\right)-t(1+s)\left(b-b_{2}\right)+\alpha \ln \frac{\alpha \rho}{\gamma \mathrm{e}}+\beta \ln \frac{\beta \rho}{\gamma \mathrm{e}}+\bar{c}_{0}
$$

and

$$
(\alpha+\beta)\left[S_{P}\left(b_{1}\right)-S_{P}\left(b_{2}\right)\right]=t(1+s)\left(b_{2}-b_{1}\right)
$$

which is equation (32) and determines $b_{1}$ as $b_{2}$ is given by relation (29).

We still have to impose conditions (ii), (iii), and (iv). First, condition (ii) ensuring no commuting within any two locations in the integrated district writes as

$$
(\alpha+\beta)\left|S_{P}^{\prime}(z)\right| \leq t(1+s), \quad z \in\left[0, b_{1}\right)
$$

that is,

$$
t>\widehat{t}_{P} \equiv \frac{\alpha+\beta}{1+s} \max _{z \in\left[0, b_{1}\right)}\left|S_{P}^{\prime}(z)\right|
$$

Second, condition (iii) says that firms outbid workers in the business district: $R(y) \geq \Psi_{r}(y) \forall y \in$ $\left[b_{1}, b_{2}\right]$. Subtracting (35) from (37), we get

$$
(\alpha+\beta)\left[S_{P}(y)-S_{P}\left(b_{2}\right)\right] \geq t(1+s)\left(b_{2}-y\right), \quad y \in\left[b_{1}, b_{2}\right]
$$

which gives

$$
t \leq \bar{t}_{P} \equiv \min _{y \in\left[b_{1}, b_{2}\right]} \frac{\alpha+\beta}{1+s} \frac{S_{P}(y)-S_{P}\left(b_{2}\right)}{b_{2}-y}
$$

Finally, condition (iv) ensuring that workers outbid firms in the residential district $\left(R(x) \geq \Psi_{b}(x)\right.$, 
$\left.\forall x \in\left[b_{2}, b\right]\right)$ gives

$$
(\alpha+\beta)\left[S_{P}\left(b_{2}\right)-S_{P}(x)\right]-t(1+s)\left(x-b_{2}\right) \geq 0, \quad x \in\left[b_{2}, b\right]
$$

which is similar to relation (39). Only signs and supports differ. Note that in the interval $\left(b_{2}, b\right), S_{P}(x)$ is convex because $G_{P}^{\prime \prime}(x)=\sigma^{2} \tau^{2} G_{P}>0$ and $\left(\ln T_{P}^{1-\sigma}(x)\right)^{\prime \prime}=0$. Since the LHS of inequality (40) is zero at $x=b_{2}$, this inequality is satisfied for all $x \in\left[b_{2}, b\right]$ if and only if

$$
(\alpha+\beta)\left[S_{P}\left(b_{2}\right)-S_{P}(b)\right] \geq t(1+s)\left(b-b_{2}\right) \geq 0
$$

Thus, inequality (40) is equivalent to

$$
t \leq \widetilde{t}_{P} \equiv \frac{\alpha+\beta}{1+s} \frac{S_{P}\left(b_{2}\right)-S_{P}(b)}{b-b_{2}}
$$

To sum up, the spatial equilibrium is given by $\left(b_{1}, b_{2}\right)$ that solve equations $(29)$ and $(32)$ and satisfy (38), (39), and (41).

Proposition (5) shows the partially integrated configuration can only exist for intermediate values of the commuting cost. It also makes explicit the condition that district borders need to satisfy. By inspection of equation (32), the commuting cost from $b_{2}$ to $b$ is to be proportional to the surplus difference between locations $b_{1}$ and $b_{2}$.

Figure 4 illustrates the shape of bid rents in a partially integrated city. Panel a) presents the case of balanced use of space, where firms and workers use the same amount of land $(s=1)$. Around the city center, firms and residents populate an integrated district where the firms' and the residents' bid rents coincide. This integrated area is surrounded by two business districts where firms outbid residents. Residential districts emerge at city edges where residents outbid firms. The condition $t \geq \widehat{t}_{P}$ ensures sufficiently high commuting cost so that workers have no incentive to commute within the integrated district. The economic intuition is similar to that prevailing in the integrated city. The condition $t \leq \widetilde{t}_{P}$ guarantees that residents outbid firms in peripheral districts. In particular it expresses the sufficient condition that firms producing at $y=b_{2}$ have no incentives to relocate production at the city edge $y=b$. Firms indeed balance the effects of such a relocation on their revenues and wage bills. On the one hand, they lose revenues at the city edge because of the lower access to their customers. On the other hand, they pay lower wages at the city edge because workers need not be compensated for long commuting. The latter effect gets smaller and is dominated by the former when commuting costs 
become lower. To sum up, partially integrated cities therefore exist only for intermediate commuting costs and, by Proposition 1, only for intermediate values of demand and input-output linkages.

We provide two Corollaries exhibiting additional properties of partially integrated equilibria. The first property relates to the contiguity and intersection of the sets of parameters supporting the integrated and the partially integrated cities. In particular, we show that the partially integrated city is a spatial equilibrium for $t=t_{I}-\delta$ and $\delta>0$ small enough.

Corollary 2 The parameter sets that support the integrated and the partially integrated cities do not intersect and are contiguous.

\section{Proof. See Appendix F.}

The second property is about the existence of partial integrated cities even when firms do not use space. The literature generally extends Alonso's (1964) model to multiple business centers located on points (e.g. Fujita, Krugman and Mori 1999). The following corollary suggests that, although insightful, this strategy does not cover the exhaustive set of urban configurations. Indeed, consider infinitesimally small business districts $(s \rightarrow 0)$. By Corollary 1 , the commuting cost interval $\left(t_{M}, t_{I}\right)$ is not empty. For such commuting costs, monocentric and integrated city structures are not spatial equilibria. In the following corollary, we show that spatial equilibria include partially integrated cities when businesses use no land.

Corollary 3 Suppose firms use an infinitely small amount of land $(s \rightarrow 0)$. Then, there exists a partially integrated equilibrium with an integrated district surrounded by two infinitely small business districts and residential districts in the periphery when commuting costs satisfy $\max \left\{t_{M}, \widehat{t}_{P}^{0}\right\} \leq t \leq$ $\min \left\{t_{I}, \bar{t}_{P}^{0}, \widetilde{t}_{P}^{0}\right\}$.

Proof. Towards this aim, we rewrite equation (32) as

$$
t=f_{s}\left(b_{1}\right) \equiv \frac{\alpha+\beta}{1+s} \frac{S_{P}\left(b_{1}\right)-S_{P}\left(b_{2}\right)}{b_{2}-b_{1}}
$$

where $b_{2}$ is given by (29). As $s \rightarrow 0$, one can verify

$$
\lim _{s \rightarrow 0} f_{s}(0)=t_{M} \text { and } \lim _{s \rightarrow 0} f_{s}(b)=t_{I}
$$

Because $t_{I}>t_{M}>0$ and $f_{s}\left(b_{1}\right)$ is continuous in $[0, b / 2]$, we can apply the intermediate value theorem. There exists at least a value of $b_{1}^{0}$ that solves $t=f_{s}\left(b_{1}\right)$ for all $t \in\left(t_{M}, t_{I}\right)$. One then computes the 
thresholds

$$
\begin{aligned}
& \widetilde{t}_{P}^{0}=(\alpha+\beta) \frac{S_{P}\left(b_{1}^{0}\right)-S_{P}(b)}{b-b_{1}^{0}} \\
& \bar{t}_{P}^{0}=-(\alpha+\beta) S_{P}^{\prime}\left(b_{1}^{0}\right) \\
& \widehat{t}_{P}^{0}=(\alpha+\beta) \max _{z \in\left[0, b_{1}^{0}\right)}\left|S_{P}^{\prime}(z)\right|
\end{aligned}
$$

Then, if $\widehat{t}_{P}^{0} \leq t \leq \min \left\{\bar{t}_{P}^{0}, \widetilde{t}_{P}^{0}\right\}$, there exists a partially integrated equilibrium with two infinitely small business districts at $y= \pm b_{1}^{0}$.

Panel b) in Figure 4 presents a second example of bid rents in a partially integrated city where the use of space is unbalanced. In such a case, firms do not use much space unlike residents and business districts have a very small spatial extent $(s=0.01)$. Additional numerical exercises show that the city configuration remains identical as $s \rightarrow 0$. In that case, the two business districts become so small that the central integrated district becomes contiguous to the peripheral residential districts. The parameters fulfill the conditions of the above corollary, which therefore determine non-empty sets of parameters. To sum up, the partially integrated city exists even in the absence of space requirement by firms. So, although very insightful, previous discussions on city subcenters concentrated on spatial points do not encompass the full set of urban configurations.

\subsection{Duocentric city}

Here, the city consists of two business districts $\mathcal{Y}=\left[-b_{3},-b_{1}\right) \cup\left(b_{1}, b_{3}\right]$ and three residential districts $\mathcal{X}=\left[-b,-b_{3}\right) \cup\left[-b_{1}, b_{1}\right] \cup\left(b_{3}, b\right]$, see Figure $1(\mathrm{~d})$. Households living in $\left[-b,-b_{3}\right)$ commute to $\left[-b_{3},-b_{2}\right)$, those living in $\left[-b_{1}, 0\right)$ to $\left[-b_{2},-b_{1}\right)$, those living in $\left[0, b_{1}\right]$ to $\left[b_{1}, b_{2}\right)$, and those living in $\left(b_{3}, b\right]$ to $\left[b_{2}, b_{3}\right)$. Given the total masses of firms and consumers, we have

$$
b_{1}=\frac{1}{s}\left(b_{2}-b_{1}\right), \quad b-b_{3}=\frac{1}{s}\left(b_{3}-b_{2}\right)
$$

The density of firms is $\mu(y)=1 / s$ on $\mathcal{Y}$ and district edges satisfy $b_{2}=(1+s) b_{1}$ and $b_{3}=b_{1}+s N / 2$. As before, we focus on $[0, b]$.

The duocentric equilibrium conditions are as follows: $(i)$ firms outbid households in the business districts $\left(\Psi_{b}(y) \geq \Psi_{r}(y)\right.$ for all $\left.y \in \mathcal{Y}\right) ;(i i)$ households outbid firms in the residential districts $\left(\Psi_{r}(x) \geq\right.$ $\Psi_{b}(x)$ for all $\left.x \in \mathcal{X}\right)$; ( $\left.i i i\right)$ the business and the residential bid rents equalize at district borders 
$\left(\Psi_{r}\left(b_{1}\right)=\Psi_{b}\left(b_{1}\right)\right.$ and $\left.\Psi_{r}\left(b_{3}\right)=\Psi_{b}\left(b_{3}\right)\right)$; and $(i v)$ the zero opportunity cost of land at the city border requiring $\Psi_{r}(b)=0$.

As of expressions (4) and (7), we define $S_{D}(z) \equiv G_{D}(z)-\ln T_{D}(z)$, where the access measure $T_{D}$ and the profit $G_{D}$ are given by

$$
\begin{aligned}
T_{D}^{1-\sigma}(z) & =\frac{1}{s}\left[\int_{-b_{3}}^{-b_{1}} e^{(1-\sigma) \tau|z-y|} \mathrm{d} y+\int_{b_{1}}^{b_{3}} e^{(1-\sigma) \tau|z-y|} \mathrm{d} y\right] \\
G_{D}(y) & =\frac{1}{\sigma s}\left[\int_{-b_{3}}^{-b_{1}} \frac{e^{-\sigma \tau|z-y|}}{T_{D}^{1-\sigma}(z)} \mathrm{d} z+\int_{b_{1}}^{b_{3}} \frac{e^{-\sigma \tau|z-y|}}{T_{D}^{1-\sigma}(z)} \mathrm{d} z\right]
\end{aligned}
$$

Proposition 6 The duocentric city is a spatial equilibrium if and only if

$$
\begin{aligned}
t & \leq \max \left(\widehat{t}_{D}, \bar{t}_{D}\right) \\
\Psi_{b}(y) & \geq \Psi_{r}(y) \text { for all } y \in \mathcal{Y}
\end{aligned}
$$

where

$$
\widehat{t}_{D} \equiv \frac{\alpha+\beta}{1+s} \frac{S_{D}\left(b_{1}+s N / 2\right)-S_{D}(0)}{(1+2 s) b_{1}-s N / 2} \text { and } \bar{t}_{D} \equiv \frac{\alpha+\beta}{1+s} \frac{S_{D}\left(b_{1}+s N / 2\right)-S_{D}(b)}{N / 2-b_{1}}
$$

with $b_{1} \in(0, N / 4]$ satisfying

$$
t\left(N-4 b_{1}\right)(1+s) s / 2=(\alpha+\beta)\left[S_{D}\left(b_{1}\right)-S_{D}\left(b_{1}+s N / 2\right)\right]
$$

Proof. As in earlier sections, workers arbitrage between the different workplaces up to their commuting cost and land rent payments. As a result, a firm located at $z$ must offer individuals a wage $w(z)$ that is as attractive as in other workplaces. Since workers commute from the left and right hand sides of the business district located on $\left(b_{1}, b_{3}\right)$, it is convenient to denote the business center of the business district by $b_{2} \in\left(b_{1}, b_{3}\right)$. This is the unique location where a firm attracts workers from each side of its location. Since the labor market divides about $y=b_{2}$, the left hand side's labor demand and supply balance so that $b_{2}-b_{1}=s b_{1}$ while its right hand side clears so that $b_{3}-b_{2}=s\left(b-b_{3}\right)$. Denoting the utility of a worker working at $b_{2}$ by $U_{2} \equiv \alpha\left[\ln (\alpha \rho / \gamma \mathrm{e})-\ln T_{D}\left(b_{2}\right)\right]+w\left(b_{2}\right)+\bar{c}_{0}$, we can write the following wage arbitrage condition for any $y \geq 0$ :

$$
\alpha\left[\ln \frac{\alpha \rho}{\gamma \mathrm{e}}-\ln T_{D}(y)\right]+w(y)+t\left|y-b_{2}\right|+\bar{c}_{0}=U_{2}
$$

so that the equilibrium wage $w(y)$ satisfies

$$
w(y)=U_{2}-\alpha\left[\ln \frac{\alpha \rho}{\gamma \mathrm{e}}-\ln T_{D}(y)\right]-t\left|y-b_{2}\right|-\bar{c}_{0}
$$


This is also the wage that a firm should pay to attract a worker when it relocates in a residential district.

By plugging the wage expression into (9) and setting $y=b_{2}$, the household's bid rent can be rewritten as

$$
\Psi_{r}(x)=U_{2}-U^{*}-t\left|x-b_{2}\right|
$$

Similarly, the firm's bid rent (10) writes as

$$
\Psi_{b}(y)=\frac{1}{s}\left[\left(\alpha \ln \frac{\alpha \rho}{\gamma \mathrm{e}}+\beta \ln \frac{\beta \rho}{\gamma \mathrm{e}}-U_{2}+\bar{c}_{0}\right)+(\alpha+\beta) S_{D}(y)+t\left|y-b_{2}\right|\right]
$$

Landlords reap the surplus of the production and consumption of each pair of individuals and firms up to the level of the individual's utility.

As before, we focus on $[0, b]$ when imposing the equilibrium conditions $(i)-(i v)$ on the bid rents from households and firms. Using the wage (44) from the wage arbitrage condition and applying it for $x>b_{3}>y>b_{2}$, one can express the relations $\Psi_{r}(b)=0$ and $\Psi_{r}\left(b_{3}\right)=\Psi_{b}\left(b_{3}\right)$ as functions of $U_{2}, U^{*}, b_{2}$ and $b_{3}$. Using the same wage (44) and applying it for $x<b_{1}<y<b_{2}$, one can also express the relation $\Psi_{r}\left(b_{1}\right)=\Psi_{b}\left(b_{1}\right)$ as function of the same variables. Therefore, conditions (iii) and (iv) together with the identities $b_{2}=(1+s) b_{1}$ and $b_{3}=b_{1}+s N / 2$ provide five equations for the five variables $U_{2}, U^{*}$, $b_{1}, b_{2}$ and $b_{3}$. The solution for $U_{2}$ and $U^{*}$ is given by

$$
\begin{aligned}
U_{2} & =\left(\alpha \ln \frac{\alpha \rho}{\gamma \mathrm{e}}+\beta \ln \frac{\beta \rho}{\gamma \mathrm{e}}+\bar{c}_{0}\right)+(\alpha+\beta) S_{D}\left(b_{1}\right)+t\left(b_{2}-b_{1}\right)-s t\left(b-2 b_{2}+b_{1}\right) \\
U^{*} & =\left(\alpha \ln \frac{\alpha \rho}{\gamma \mathrm{e}}+\beta \ln \frac{\beta \rho}{\gamma \mathrm{e}}+\bar{c}_{0}\right)+(\alpha+\beta) S_{D}\left(b_{1}\right)-(1+s) t\left(b-2 b_{2}+b_{1}\right)
\end{aligned}
$$

while $b_{1}$ solves (43).

The equilibrium inequality condition $(i i) \Psi_{r}(x) \geq \Psi_{b}(x)$ for all $x \in \mathcal{X}$, writes as

$$
\Psi_{r}(x)-\Psi_{b}(x)=U_{2}-U^{*}-\frac{1}{s}\left[\alpha \ln \frac{\alpha \rho}{\gamma \mathrm{e}}+\beta \ln \frac{\beta \rho}{\gamma \mathrm{e}}-U_{2}+\bar{c}_{0}+(\alpha+\beta) S_{D}(x)\right]-\frac{1+s}{s} t\left|x-b_{2}\right| \geq 0
$$

where $S_{D}(x)$ can be shown to be convex on the intervals $\left(0, b_{1}\right)$ and $\left(b_{3}, b\right): S_{D}^{\prime \prime}=G_{D}^{\prime \prime}-\frac{1}{1-\sigma}\left(\ln T_{D}^{1-\sigma}\right)^{\prime \prime}$ where $G_{D}^{\prime \prime}=\sigma^{2} \tau^{2} G_{D}>0$ and $\left(\ln T_{D}^{1-\sigma}\right)^{\prime \prime}=\left[\left(T_{D}^{1-\sigma}\right)^{\prime \prime} T_{D}^{1-\sigma}-\left(T_{D}^{1-\sigma}\right)^{\prime^{2}}\right] / T_{D}^{2(1-\sigma)}$; the last expression is equal to 0 on $\left(b_{3}, b\right)$ and to $\left[2(\sigma-1) \tau /\left(s T_{D}^{1-\sigma}\right)\right]^{2} \int_{-b_{3}}^{-b_{1}} e^{(1-\sigma) \tau(y-z)} \mathrm{d} z \int_{-b_{3}}^{-b_{1}} e^{(1-\sigma) \tau(z-y)} \mathrm{d} z>0$ on $\left(0, b_{1}\right)$. Then, $\Psi_{r}(x)-\Psi_{b}(x)$ is concave on those two intervals. Since $\Psi_{r}\left(b_{1}\right)-\Psi_{b}\left(b_{1}\right)=0$, we have $\Psi_{r}(x)-$ $\Psi_{b}(x) \geq 0$ for all $x \in\left(0, b_{1}\right)$ if and only if $\Psi_{r}(0)-\Psi_{b}(0) \geq 0$, which can be rewritten as $t \leq$ $\widehat{t}_{D} \equiv(\alpha+\beta)\left[S_{D}\left(b_{3}\right)-S_{D}(0)\right] /\left[\left(2 b_{2}-b_{3}\right)(1+s)\right]$. Similarly, since $\Psi_{r}\left(b_{3}\right)-\Psi_{b}\left(b_{3}\right)=0$, we have 
$\Psi_{r}(x)-\Psi_{b}(x) \geq 0$ for all $x \in\left(b_{3}, b\right)$ if and only if $\Psi_{r}(b)-\Psi_{b}(b) \geq 0$, which can be rewritten as $t \leq \bar{t}_{D} \equiv(\alpha+\beta)\left[S_{D}\left(b_{3}\right)-S_{D}(b)\right] /\left[\left(b-b_{3}\right)(1+s)\right]$. This means that condition $(i i)$ reduces to $t \leq \max \left(\widehat{t}_{D}, \bar{t}_{D}\right)$. The equilibrium inequality condition $(i)$ remains as it is: $\Psi_{b}(y) \geq \Psi_{r}(y)$ for all $y \in \mathcal{Y}$.

Finally, it can easily be shown that $b_{1}$ should not exceed $N / 4$. Otherwise, the residential bid rent would be negative at $x=0$.

To understand, the equilibrium condition in Proposition (43), we rewrite it as

$$
t\left(b_{3}-b_{2}\right)-t\left(b_{2}-b_{1}\right)=\frac{\alpha+\beta}{(1+s) s}\left[S_{D}\left(b_{1}\right)-S_{D}\left(b_{3}\right)\right]
$$

The LHS corresponds to the difference in the commuting cost to workplace $b_{2}$ from district borders $b_{3}$ and $b_{1}$, while the RHS is proportional to the surplus difference between locations $b_{3}$ and $b_{1}$. This means that these commuting costs and surplus differences should balance.

Figure 5 illustrates the shape of bid rents in a duocentric city. Residential districts are located at the city center and peripheries where the residents' bid rent (in blue) is above the firms' bid rent curve (in red). In between these residential districts, two business districts gather firms outbidding residents. Panel a) and b) of Figure 5 show two different duocentric city structures for the same set of economic parameters, for which equation (43) accepts two solutions. It can be checked on Figure 5 show that $\Psi_{b}(y) \geq \Psi_{r}(y)$ for all $y$ in the business districts. So, this means that multiple duocentric equilibria can arise.

In Proposition 6, the endogenous border $b_{1}$ is the solution to (43), which accepts no closed-form solution. Proposition 6 also imposes the bid rent condition $\Psi_{b}(y) \geq \Psi_{r}(y)$, for any location $y$ in the business districts $\mathcal{Y}$. As the purpose will be to determine the duocentric configurations by use of numerical computations, we shall replace the condition $\Psi_{b}(y) \geq \Psi_{r}(y)$, for all $y \in \mathcal{Y}$, by the necessary condition $\Psi_{b}\left(b_{2}\right) \geq \Psi_{r}\left(b_{2}\right)$, which is equivalent to $t \leq \widetilde{t}_{D}$ where

$$
\widetilde{t}_{D} \equiv \frac{\alpha+\beta}{1+s} \frac{S_{D}\left(b_{2}\right)-S_{D}\left(b_{1}\right)}{b_{2}-b_{1}}
$$

Note that we did not find any example where this condition was not also sufficient. So, the threshold value of the commuting cost is given by

$$
t \leq t_{D}=\max \left(\widehat{t}_{D}, \bar{t}_{D}, \widetilde{t}_{D}\right)
$$




\section{$5 \quad$ Numerical analysis}

The theoretical analysis of urban structures in the previous section allows us to determine and study the nature of city structures in terms of economic parameters. Given the complex nature of duocentric and partially integrated configurations, we determine them by numerical computations. This is done in two steps. First, given some fixed parameter values, district borders are determined by solving (43) and (42) for the duocentric configuration (resp. (32) for the partially integrated configuration). Note that up to two roots are found in either case. Second, additional restrictions reflecting rent conditions at district borders are imposed. The role of these restrictions is to retain only the candidates that actually lead to an equilibrium configuration.

Equilibrium city structures Figure 6 gathers the central results of our numerical analysis. It presents the equilibrium structures derived in Propositions 3, 4, 5, and 6 in the plane of transport and commuting costs $(\tau, t)$ for fixed values of other parameters, i.e. $\alpha=\beta=\gamma=s=1$ and $\sigma=3$. The figure displays the monocentric " $m$ ", the integrated " $i$ ", the partially integrated " $p$ ", and the duocentric " $d$ " configurations. The concatenation of several letters indicates the presence of multiple equilibria with different urban structures for the same set of parameters. For instance, "mdd" indicates the co-existence of a monocentric equilibrium and two different duocentric equilibria. For some parameter configurations, up to four equilibria can co-exist (see "dmpp" at the center of Figure 6). In Section 2, existence of a spatial equilibrium was proved. Indeed, numerical computations always deliver at least an equilibrium for any point in $(\tau, t)$ plane. This contrasts with Fujita and Ogawa (1982) where some parameter configurations were shown to support no equilibrium.

Figure 6 displays the curve corresponding the threshold $t_{M}$ below which parameters support the monocentric city. The monocentric city arises only if commuting costs are relatively low. By definition of $t_{M}$, residents and businesses make the same land bid at the city center for any value of $t$ lying on the curve $t_{M}$. For slightly higher commuting costs -and as long as transport costs are low enough-, residents are willing to match the firms' bid rent at the city center. Some residents and firms can then gather at the city center, while many other workers are still willing to commute. Hence the curve $t_{M}$ refers to a transition between the monocentric and the partially integrated configurations.

Figure 6 also displays the curve corresponding to the threshold $t_{I}$ above which parameters support the integrated city. In the integrated city, residents work and reside in the same location. By definition 
of threshold $t_{I}$, when commuting costs equate $t_{I}$, some individuals are indifferent between working at their residence location and commuting to a firm located further away. For commuting costs slightly below $t_{I}$, residents located at the city border have an incentive to commute and choose a workplace between the city center and the city border, so that a partially integrated city structure emerges. Hence the curve $t_{I}$ refers to a transition between the the partially integrated and the integrated configurations.

The partially integrated configuration exists only for intermediate commuting costs. Consider such a partially integrated structure. When the commuting cost rise becomes too high, the business centers and the residential peripheries shrink, and ultimately vanish when the commuting cost reaches the threshold $t_{I}$. Hence the partially integrated configuration converges smoothly to the integrated city. For low transport costs (i.e., when only one partially integrated equilibrium exists), the two business centers get closer to each other to finally join each other as the commuting cost approaches $t_{M}$ from above: the partial integrated structure then converges smoothly to the monocentric configuration. For higher transport costs (i.e., when the partially integrated and the monocentric cities co-exist), one partially integrated configuration converges continuously to the monocentric structure as the commuting cost approaches $t_{M}$ from below.

Duocentric cities are equilibria only for high transport costs and intermediate commuting costs (see the right-hand side of Figure 6). Indeed, when shipping goods are costly, firms benefit from clustering their production activities in two separate business centers so as to decrease the average distance to suppliers and consumers. Consider commuting costs rising from zero (i.e., a rising path in the right-hand side of Figure 6). When commuting costs are very low, workers accept long journeys to their workplace and benefit from a concentrated business district, i.e. the monocentric pattern "m". However, when commuting costs reach intermediate values, an urban structure with two activity centers becomes beneficial to them because long commutes can be avoided. When this happens, two duocentric configurations emerge (see transition from "m" to "mdd" in Figure 6). As commuting costs keep on increasing, one duocentric configuration persists while the other disappears (transition from "dmd" to "dm"). Finally, for even higher commuting costs, the remaining duocentric city pattern ceases to exist as households want to locate closer to their workplace, which gives rise to a transition to a partially integrated configuration. 
Multiple equilibria Figure 6 also illustrates the multiplicity of equilibria. In many regions of the parameter space, a unique equilibrium emerges (see "m", "i", "p" and "d"). There are also large sets of parameters for which several structures among the monocentric, the duocentric, or the partially integrated configurations co-exist. Note that all structures can co-exist for a narrow set of parameter values. The fully integrated structure cannot co-exist with any other configuration. As mentioned in the above paragraph, multiple equilibria can involve different urban structures of the same type: either two partially integrated structures like in "mpp" and "dmpp" or two duocentric structures like in "dmd" and "mdd". This can only happen for intermediate commuting costs. For higher commuting costs, one of the two equilibria always disappears. It is interesting to describe how two equilibria of the same type emerge. When commuting costs increase, it can be shown that the transition from " $\mathrm{m}$ " to "mpp" (resp. from "m" to "mdd") implies that the monocentric city keeps the same structure while a partially integrated city (resp. a duocentric city) initially emerges and immediately separates into two distinct partially integrated structures (resp. a duocentric) with business centers evolving at different locations.

Welfare ranking Importantly, the ordering of letters in Figure 6 indicates the ranking of equilibria in terms of aggregate welfare (i.e., the sum of residents' utility plus aggregate land rents). For instance, the ordering "mdd" indicates that the monocentric configuration has the highest welfare while the two duocentric configurations have lower and distinct welfare values. The welfare ranking can be obtained from Figure 7, which plots the aggregate welfare in terms of the transport cost for nine values of commuting cost. The top left panel shows that for $t=0.2$, the aggregate welfare falls with higher transport costs as they indeed reduce consumption and therefore profits, wages and utility. The panel shows continuous welfare transitions between the integrated, the partially integrated and the monocentric cities as transport costs rise. For high transport costs, two duocentric configurations emerge but yield lower welfare levels. The next two top panels show that for higher commuting costs $t=0.32$ and 0.56 respectively, duocentric structures can bring higher welfare. The central panel illustrates equilibria and the welfare ranking for $t=0.64$ : two partially integrated equilibria rank below the monocentric city equilibrium. In the next panels, commuting cost are so high that the monocentric configuration is not part of equilibria. It can be observed that the duocentric city abruptly emerges as commuting costs increase and yields a higher aggregate welfare. 
Population growth Finally, we look at the impact of urbanization on spatial structures by considering gradual increases in the city size $N$ for given commuting and transport costs. According to our numerical analysis, three cases arise depending on transport costs $\tau$.

(i) For low transport costs $\tau$, transitions are smooth. The configuration is initially monocentric for small $N$ (i.e. $t<t_{M}$ ), then it becomes partially integrated for intermediate $N$ (i.e. $t_{M}<t<t_{I}$ ).

(ii) For intermediate transport $\operatorname{costs} \tau$, the configuration is initially monocentric. Then it bifurcates into either a partially integrated or duocentric city. These patterns are quite different from the monocentric city so that the transition is discontinuous, the resulting path being undetermined. In the case of a duocentric city, it will partially integrate at a later stage, and will finally approach full integration.

(iii) For large transport costs $\tau$, the configuration is initially monocentric. When it bifurcates, it becomes a duocentric city. Then, it becomes partially integrated, and finally fully integrated. Figure 8 plots this case with $\tau=3$ and $t=0.7$ for an increasing population $N$. It shows that the configuration is initially monocentric (black). When it breaks, it becomes duocentric (red), and then partially integrated (blue).

These results show that transitions are continuous (resp. discontinuous) for small (resp. large) transport costs. This reflects the fact that when transport costs are large, firms can gain more when firms locate in several business districts.

\section{Conclusion}

We have studied urban structures driven by demand and vertical linkages in the presence of increasing returns to scale, which in contrast to the existing literature on the topic, are internal to the firm. Existence of equilibrium has been proved and an invariance result has been obtained regarding the equivalent role of more intense demand and vertical linkages and lower commuting costs. Various urban configurations can emerge in equilibrium exhibiting a monocentric, an integrated, a duocentric, or a partially integrated city structure. We have discussed the role of commuting and transport costs, intensities of demand/vertical linkages, and urbanization in affecting these patterns. We have finally presented a numerical approach to discuss the emergence of multiple equilibria. 


\section{Appendix A: Equilibrium conditions}

Let $\mathcal{B}=[-b, b], b \in \mathbb{R}_{0}^{+}$, be the support of the city. The densities of residents and firms are given by $\lambda: \mathcal{B} \rightarrow[0,1]$ and $\mu: \mathcal{B} \rightarrow[0,1 / s]$. The land market clearing imposes that $\lambda+s \mu=1$ while labor market clearing imposes that the mass of workers is equal to mass of firms $\int_{\mathcal{B}} \lambda=\int_{\mathcal{B}} \mu=N$. With the city border $b \equiv N(1+s) / 2$, every worker and every firm find a job match. So, we just need to impose $\int_{\mathcal{B}} \mu=N$.

While worker's bid rent is the maximum bid a worker makes on a parcel of land as of (13), the firm's bid rent is the maximum bid that it can make on land as of (14). The necessary and sufficient conditions for spatial equilibrium are given by identities (16) and (17). Remind that $S(y)=G(y)-\ln T(y)$, where

$T(y)=\left[\int_{\mathcal{B}} e^{(1-\sigma) \tau|z-y|} \mu(z) \mathrm{d} z\right]^{\frac{1}{1-\sigma}}$ and $G(y)=\frac{1}{\sigma} \int_{\mathcal{B}} \frac{e^{-\sigma \tau|z-y|}}{T^{1-\sigma}(z)} \mu(z) \mathrm{d} z$. Note that $G, T$ and therefore $S:$ $\mathcal{B} \rightarrow \mathbb{R}^{+}$are integrals and therefore continuous functions of $y$. Functions $G$ and $T$ are also strictly positive because their integrands are positive as $\mu$ has positive measure.

No cross commute At every location, the flow of commuters is unidirectional. The proof can be found in Ogawa and Fujita (1980, Property 1). The authors show that workers commuting in opposite directions have no incentives to cross each other in equilibrium.

City edges Spatial equilibrium involves workers residing at city edges. Suppose this is not the case: $\lambda(b)=0$ and $\mu(b)=1 / s$. Then, there would be workers at $x<b$ facing a positive land rent and commuting to a firm located at $b$. These workers would benefit from relocating to $b+\delta$, with $\delta>0$ infinitely small, because of a zero land rent and an infinitely small commuting cost $t \delta$, which violates equilibrium. The same argument applies at $x=-b$.

More generally, spatial equilibrium involves more residents than firms at city edges. Otherwise, $\lambda(b)<\mu(b)$, and the above argument still applies as there would be workers at $x<b$ facing a positive land rent and commuting to a firm in $b$, who would benefit from relocating to $b+\delta$, with $\delta>0$ infinitely small.

Lemma 1 City edges host more residents than firms: $\lambda>0, \lambda \geq \mu$ for $z= \pm b$.

Land rent continuity The bid rents $\Psi_{r}$ and $\Psi_{b}$ are the maximum values of programs (13) and (14), which involve not only the continuous functions $S$ and $|\cdot|$, but also the land rent function $R$ which 
is not a priori continuous. Therefore, the Maximum Theorem cannot be applied in order to show the continuity of bid rents $\Psi_{r}$ and $\Psi_{b}$. Instead, the continuity of bid rents is shown by the fact that they are linear functions of the land rent $R$.

Lemma 2 The bid rents $\Psi_{r}$ and $\Psi_{b}$ in (13) and (14) are continuous functions $\mathcal{B} \rightarrow \mathbb{R}$. So $\Psi_{r}-\Psi_{b}$ and $R=\max \left\{\Psi_{r}, \Psi_{b}, 0\right\}$ are also continuous functions $\mathcal{B} \rightarrow \mathbb{R}$.

Proof. Indeed, each bid rent can be written as the function $\Psi(x)=\max _{z} f_{1}(x)+f_{2}(z)+f_{3}(x-z)$, where $f_{1}: \mathcal{B} \rightarrow \mathbb{R}$ and $f_{3}:[-2 b, 2 b] \rightarrow \mathbb{R}$ are continuous functions and $f_{2}: \mathcal{B} \rightarrow \mathbb{R}$ is not necessarily a continuous function. For $\Psi=\Psi_{r}$, take $f_{1}=0, f_{2}=\alpha \ln \frac{\alpha \rho}{\gamma \mathrm{e}}+\beta \ln \frac{\beta \rho}{\gamma \mathrm{e}}+(\alpha+\beta) S+\bar{c}_{0}-U^{*}-s R$ and $f_{3}(x-z)=-t|x-z|$. For $\Psi=\Psi_{b}$, take $f_{1}=\left(\alpha \ln \frac{\alpha \rho}{\gamma \mathrm{e}}+\beta \ln \frac{\beta \rho}{\gamma \mathrm{e}}+(\alpha+\beta) S+\bar{c}_{0}-U^{*}\right) / s, f_{2}=-R / s$ and $f_{3}(x-z)=-t|x-z| / s$. Remember that $S$ is a continuous function. In contrast, the land rent $R$ is not a priori a continuous function. Let $y$ and $y^{\prime}$ be the maximizers of $\Psi$ respectively at $x$ and $x^{\prime}$. By the definition of a maximum, we have $\Psi(x)=f_{1}(x)+f_{2}(y)+f_{3}(x-y) \geq f_{1}(x)+f_{2}\left(y^{\prime}\right)+f_{3}\left(x-y^{\prime}\right)$ and $\Psi\left(x^{\prime}\right)=f_{1}\left(x^{\prime}\right)+f_{2}\left(y^{\prime}\right)+f_{3}\left(x^{\prime}-y^{\prime}\right) \geq f_{1}\left(x^{\prime}\right)+f_{2}(y)+f_{3}\left(x^{\prime}-y\right)$. Using the conditions, we have

$$
f_{3}\left(x-y^{\prime}\right)-f_{3}(x-y) \leq f_{2}(y)-f_{2}\left(y^{\prime}\right) \leq f_{3}\left(x^{\prime}-y^{\prime}\right)-f_{3}\left(x^{\prime}-y\right)
$$

Then, we compute the difference

$$
\Psi(x)-\Psi\left(x^{\prime}\right)=f_{1}(x)-f_{1}\left(x^{\prime}\right)+f_{2}(y)-f_{2}\left(y^{\prime}\right)+f_{3}(x-y)-f_{3}\left(x^{\prime}-y^{\prime}\right)
$$

and therefore

$$
f_{2}(y)-f_{2}\left(y^{\prime}\right)=\Psi(x)-\Psi\left(x^{\prime}\right)-\left(f_{1}(x)-f_{1}\left(x^{\prime}\right)\right)-\left(f_{3}(x-y)-f_{3}\left(x^{\prime}-y^{\prime}\right)\right)
$$

Plugging this in (45), we get

$$
f_{1}(x)-f_{1}\left(x^{\prime}\right)+f_{3}\left(x-y^{\prime}\right)-f_{3}\left(x^{\prime}-y^{\prime}\right) \leq \Psi(x)-\Psi\left(x^{\prime}\right)=f_{1}(x)-f_{1}\left(x^{\prime}\right)-f_{3}\left(x^{\prime}-y\right)+f_{3}(x-y)
$$

Then, because $f_{1}$ and $f_{3}$ are continuous functions, $\lim _{x^{\prime} \rightarrow x}\left|f_{1}(x)-f_{1}\left(x^{\prime}\right)\right|=0$ and $\lim _{y^{\prime} \rightarrow y} \mid f_{3}\left(x-y^{\prime}\right)-f_{3}\left(x^{\prime}-y^{\prime}\right.$ $\lim _{x^{\prime} \rightarrow x}\left|f_{3}\left(x^{\prime}-y\right)-f_{3}(x-y)\right|=0$, we have that $\lim _{x^{\prime} \rightarrow x}\left|\Psi(x)-\Psi\left(x^{\prime}\right)\right|=0$.

Commuting flows Let $n: \mathcal{B} \rightarrow \mathbb{R}$ be the number of commuters flowing in the right hand direction (i.e. toward positive $x$ ). Since there is no resident and no firm at the left of $x=-b$ and at the right of 
$x=b$, we have $n( \pm b)=0$. This flow increases with the mass of residents who start their commuting trip in the right direction and decreases with the mass of firms that hire commuters located on their left: $\dot{n}=\lambda-\mu$. Given the definition of spatial equilibrium, we have

$$
\dot{n} \in\left\{\begin{array}{ccc}
\{1\} & \text { if } & \Psi_{r}>\Psi_{b} \\
{\left[-\frac{1}{s}, 1\right]} & \text { if } & \Psi_{r}=\Psi_{b} \\
\left\{-\frac{1}{s}\right\} & \text { if } & \Psi_{r}<\Psi_{b}
\end{array}\right.
$$

Given the absence of cross commuting, a location is a 'source' of commuters if $\dot{n}>0$ and a 'sink' of commuters if $\dot{n}<0$. The flow of commuters points to the right direction $(x<y)$ when $n>0$ and to the left direction $(x>y)$ if $n<0$. By the first fundamental theorem of calculus, the flow of commuters $n(z)=\int_{-b}^{z} \dot{n} \mathrm{~d} z$ is a continuous function. At city edges, because $\lambda \geq \mu$ at $z= \pm b$, we have $\dot{n}( \pm b) \geq 0$. Also, when $\dot{n}( \pm b)>0$, the function $\dot{n}$ is equal to 0 at least twice in order to satisfy $n(b)=\int_{-b}^{b} \dot{n} \mathrm{~d} z=0$.

It is useful to introduce some terminology about the sets of sources and sinks of commuters moving to right or left direction. Let the set of sources $\mathcal{S}^{\kappa}=\{z \in \mathcal{B}: \dot{n}>0$ and $\kappa n>0\}$ and the set of sinks (destinations) $\mathcal{D}^{\kappa}=\{z \in \mathcal{B}: \dot{n}<0$ and $\kappa n>0\}$ where $\kappa=1$ if commuters flow to the right direction and $\kappa=-1$ otherwise. For example, $\mathcal{S}^{1}$ includes source locations of commuters flowing to the right direction and $\mathcal{S}^{-1}$ those flowing to the left direction. Obviously, commuters departing from $\mathcal{S}^{\kappa}$ and arriving in $\mathcal{D}^{\kappa}$ flow to the right direction (resp. the left direction) if $\kappa=1$ (resp. - 1 ). Locations that are neither origin nor destination of commuters define the set $\mathcal{P}=\{z \in \mathcal{B}: \dot{n}=0\}$. Locations that involve a change in commuting direction are denoted by the set $\mathcal{Q}=\{z \in \mathcal{B}: n=0$ and $\dot{n} \neq 0\}$. Because $n$ is a continuous function and either $\dot{n}>0$ or $\dot{n}<0$ for $z \in \mathcal{Q}$, any location $z \in \mathcal{Q}$ has zero measure, i.e. it is an isolated point. Finally, note that those definitions determine six disjoint sets: $\mathcal{D}^{+1}, \mathcal{D}^{-1}, \mathcal{S}^{1}, \mathcal{S}^{-1}, \mathcal{P}$ and $\mathcal{Q}$.

We now study the land rent properties on these sets.

Wage arbitrage in $\mathcal{S}^{\kappa} \cup \mathcal{D}^{\kappa}$. Consider two disjoint, open and convex intervals, $\mathcal{S}^{\prime} \subseteq \mathcal{S}^{\kappa}$ and $\mathcal{D}^{\prime} \subseteq \mathcal{D}^{\kappa}$, such that (i) workers residing in $\mathcal{S}^{\prime}$ commute to firms located in $\mathcal{D}^{\prime}$ and labor flows in the same direction within each interval $\left(S^{\prime} \cap \mathcal{D}^{\prime}=\varnothing\right)$. Since $n(x)$ and $n(y)$ have the same sign for $x \in \mathcal{S}^{\prime}$ and $y \in \mathcal{D}^{\prime}$, one readily checks that $|x-y|=(y-x) \operatorname{sign}(n(x))=y \operatorname{sign}(n(y))-x \operatorname{sign}(n(x))$ where $\operatorname{sign}(n)=1,0,-1$ depending on whether $n$ is positive, zero, or negative. Since $n$ has the same sign in each interval, each function $\operatorname{sign}(n)$ is a constant. The first order conditions for the maximum 
problems (13) and (14) are given by

$$
\begin{aligned}
(\alpha+\beta) \dot{S}-t \operatorname{sign}(n)-s \dot{R} & =0, \quad y \in \mathcal{D}^{\prime} \\
t \operatorname{sign}(n)-\dot{R} & =0, \quad x \in \mathcal{S}^{\prime}
\end{aligned}
$$

While the first identity includes only residents irrespective of their workplaces, the second one involves only firms irrespective of workers' residences. These identities apply for any infinitesimal interval where workers start to commute in the same direction and firms hire from the same side of the city. Since these conditions hold for all $\mathcal{S}^{\prime} \subseteq \mathcal{S}^{\kappa}$ and $\mathcal{D}^{\prime} \subseteq \mathcal{D}^{\kappa}$, they hold a.e. in $\mathcal{S}^{\kappa}$ and $\mathcal{D}^{\kappa}$, where a.e. (almost everywhere) means for all points except on a zero-measure set.

Lemma 3 The land bid rent differential is given by

$$
\dot{\Psi}_{b}-\dot{\Psi}_{r}=\frac{1}{s}(\alpha+\beta) \dot{S}-\frac{1+s}{s} \operatorname{tsign}(n), \quad \text { a.e. } z \in \mathcal{S}^{\kappa} \cup \mathcal{D}^{\kappa}
$$

Proof. Consider again the two disjoint, open and convex intervals $\mathcal{S}^{\prime} \subseteq \mathcal{S}^{\kappa}$ and $\mathcal{D}^{\prime} \subseteq \mathcal{D}^{\kappa}$. Then, conditions (47) and (48) imply that the equilibrium land rent $R$ is equal to workers' and firms' bid rents. Applying the envelope theorem to (13) and (14) yields

$$
\begin{aligned}
& \dot{\Psi_{r}}=t \operatorname{sign}(n), \quad x \in \mathcal{S}^{\prime} \\
& \dot{\Psi_{b}}=\frac{1}{s}[(\alpha+\beta) \dot{S}-t \operatorname{sign}(n)], \quad y \in \mathcal{D}^{\prime}
\end{aligned}
$$

We construct the 'highest losing' bids $\Psi_{r}$ on $\mathcal{D}^{\prime}$ and $\Psi_{b}$ on $\mathcal{S}^{\prime}$. We first look at the set $\mathcal{S}^{\prime}$. Because workers depart from their residence $x \in \mathcal{S}^{\prime}$ to work, they must reside on $\mathcal{S}^{\prime}$ and win the land market competition. Because workers reside in $\mathcal{S}^{\prime}$, her bid $\Psi_{r}$ must be equal to the equilibrium land price $R$. Consider then a firm that would produce at $x \in \mathcal{S}^{\prime}$ and hire a resident at $x^{\prime} \in \mathcal{S}^{\prime}$. Because of (48), any resident in $\mathcal{S}^{\prime}$ has the same incentive to work in the firm. So, we can say that the firm hires a worker residing at $x^{\prime}=x$. The firm's highest (losing) bid $\Psi_{b}$ is given by (14) evaluated at $y=x$. Using this, the bid rent differential writes as

$$
\Psi_{b}-\Psi_{r}=\frac{1}{s}\left[\alpha \ln \frac{\alpha \rho}{\gamma \mathrm{e}}+\beta \ln \frac{\beta \rho}{\gamma \mathrm{e}}+(\alpha+\beta) S+\bar{c}_{0}-U^{*}\right]-\frac{1+s}{s} R
$$

Differentiating this expression and using (48), we have the bid rent differential

$$
\dot{\Psi}_{b}-\dot{\Psi}_{r}=\frac{1}{s}(\alpha+\beta) \dot{S}-\frac{1+s}{s} t \operatorname{sign}(n), \quad z \in \mathcal{S}^{\prime}
$$


which holds for any $\mathcal{S}^{\prime}$ in $\mathcal{S}^{\kappa}$, and thus for any measurable set in $\mathcal{S}^{\kappa}$.

We then look at the set $\mathcal{D}^{\prime}$. Because $\mathcal{D}^{\prime}$ hosts firms, it must be that $\Psi_{b}=R$. Consider a resident who would choose to reside at $y \in \mathcal{D}^{\prime}$ and be hired by a firm in $\mathcal{D}^{\prime}$. Because of (47), the worker is indifferent to any firm producing at $y^{\prime} \in \mathcal{D}^{\prime}$, so that we can focus on the case where the hiring firm produces at $y^{\prime}=y$. The worker's highest (losing) bid is given by (13) evaluated at $x=y$. The bid rent difference writes as

$$
\Psi_{b}-\Psi_{r}=-\left(\alpha \ln \frac{\alpha \rho}{\gamma \mathrm{e}}+\beta \ln \frac{\beta \rho}{\gamma \mathrm{e}}\right)-(\alpha+\beta) S-\bar{c}_{0}+U^{*}+(1+s) R
$$

Differentiating this expression and using (48), we get the bid rent differential

$$
\dot{\Psi}_{b}-\dot{\Psi}_{r}=\frac{1}{s}(\alpha+\beta) \dot{S}-\frac{1+s}{s} t \text { signn }, \quad y \in \mathcal{D}^{\prime}
$$

which holds for any $\mathcal{D}^{\prime}$ in $\mathcal{D}^{\kappa}$, and thus for any measurable set in $\mathcal{D}^{\kappa}$.

Wage arbitrage in $\mathcal{P}$. Consider a location $z \in \mathcal{P}$, which is neither a source nor a sink of commuters. Since there is no empty hinterland, this location must host both workers and firms, so that land market clearing imposes $R=\Psi_{b}=\Psi_{r}$. So,

$$
R=\frac{1}{1+s}\left[\alpha \ln \frac{\alpha \rho}{\gamma \mathrm{e}}+\beta \ln \frac{\beta \rho}{\gamma \mathrm{e}}+(\alpha+\beta) S+\bar{c}_{0}-U^{*}\right], \quad z \in \mathcal{P}
$$

Furthermore, let $\stackrel{\circ}{\mathcal{P}}$ be the interior set of $\mathcal{P}$ so that $z \in \stackrel{\circ}{\mathcal{P}}$ is not an isolated point in $\mathcal{P}$. Then, we have

$$
\dot{R}=\dot{\Psi}_{r}=\dot{\Psi}_{b}=\frac{1}{1+s}(\alpha+\beta) \dot{S}, \quad z \in \stackrel{\circ}{\mathcal{P}}
$$

and

$$
\dot{\Psi}_{b}-\dot{\Psi}_{r}=0, \quad z \in \stackrel{\circ}{\mathcal{P}}
$$

Bid rent differential at city borders. The zero opportunity cost of land imposes $R=0$ at the city border $z=-b$. By Lemma 1 , there must be workers with residences at $z=-b$ so that the highest bid is that of workers: $\Psi_{r}=R=0$ at $z=-b$. The edge location $z=-b$ also belongs to the set $\mathcal{S}^{1}$ since $\lambda(-b)>0$ and workers there cannot commute to the left direction. We can therefore apply (49) and get the land rent differential

$$
\Psi_{b}-\Psi_{r}=\Psi_{b}=\frac{1}{s}\left[\alpha \ln \frac{\alpha \rho}{\gamma \mathrm{e}}+\beta \ln \frac{\beta \rho}{\gamma \mathrm{e}}+(\alpha+\beta) S+\bar{c}_{0}-U^{*}\right], \quad z=-b
$$


Equilibrium condition We summarize the above results as follows. Let us define $\phi: \mathcal{B} \rightarrow \mathbb{R}$,

$$
\phi(z)=\frac{1}{s}(\alpha+\beta) \dot{S}(z)-\frac{1+s}{s} t \operatorname{sign}(n(z))
$$

Note that $\dot{n}$ and $\phi$ may not be continuous in $z$. However $\phi$ is bounded because $\dot{S}$ is bounded.

A spatial equilibrium is defined by the rent differential function $\Psi_{b}-\Psi_{r}$ and the commuting function $n$ such that $\Psi_{b}-\Psi_{r}$ is continuous everywhere on $\mathcal{B}, \Psi_{b}-\Psi_{r}=\Psi_{b}$ and $\dot{n} \geq 0=n$ at $z \in\{-b, b\}$, and

$$
\begin{array}{ccc}
z \in \mathcal{S}^{\kappa} \cup \mathcal{D}^{\kappa} & \Rightarrow & \dot{\Psi}_{b}-\dot{\Psi}_{r}=\phi(z) \text { a.e. } \\
z \in \mathcal{P} & \Rightarrow & \dot{\Psi}_{b}-\dot{\Psi}_{r}=0 \text { a.e. }
\end{array}
$$

Remind that $\mathcal{Q}$ has zero measure and therefore is not affected by the above implications.

Lemma 4 A spatial equilibrium is such that

$$
\begin{aligned}
z \in \mathcal{S}^{\kappa} \cup \mathcal{D}^{\kappa} & \Longleftrightarrow \phi(z) \neq 0 \text { a.e. } \\
z \in \mathcal{P} & \Longleftrightarrow \phi(z)=0 \text { a.e. }
\end{aligned}
$$

Note that any equilibrium commuting function $n$ has two possible patterns: it is either such that $\dot{n}=n=\phi=0, \forall z \in \mathcal{B}$ (fully integrated city) or such that the city space is partitioned with $\dot{n}>0$ for some source locations $z$ and $\dot{n}<0$ for some $\operatorname{sink}$ locations $z$. In the latter case, we have $\phi>0$ for some $z$ and $\phi<0$ for some other $z$. This means that $\phi$ always accepts a zero. Let $Z$ be the set of zeroes or changes in the sign of $\phi, Z=\left\{z \in \mathcal{B}: \lim _{\varepsilon \rightarrow 0} \phi(z+\varepsilon) \cdot \phi(z-\varepsilon) \leq 0\right\}$.

Then, the spatial equilibrium is given by the function $n: \mathcal{B} \rightarrow \mathbb{R}$, which is the solution to

$$
\dot{n}(z) \in\left\{\begin{array}{ccc}
\{1\} & \text { if } & \Phi(z)<0 \\
{\left[-\frac{1}{s}, 1\right]} & \text { if } & \Phi(z)=0 \\
\left\{-\frac{1}{s}\right\} & \text { if } & \Phi(z)>0
\end{array}\right.
$$

with $\dot{n} \geq 0=n$ at $z= \pm b$ and $\Phi(z) \equiv \int_{\widehat{z} \in Z}^{z} \phi(x) \mathrm{d} x$. Using $n(x)=\int_{-b}^{x}(\lambda-\mu) \mathrm{d} z$ yields condition $(16)$ in the text.

Invariance To check invariance, one can replace $t$ and $(\alpha+\beta)$ by $\widetilde{t}=m t$ and $(\widetilde{\alpha}+\widetilde{\beta})=m(\alpha+\beta)$, $m \in \mathbb{R}_{0}$. This yields $\widetilde{\phi}(n)=m \phi(n)$, which gives the same set of roots $\widehat{Z}=\{z \in \mathcal{B}: \widetilde{\phi}(n)=0\}=$ $\{z \in \mathcal{B}: \phi(n)=0\}$. As a result, the sign of $\widetilde{\Phi}(n)$ is the same as that of $\Phi(n)$, and thus, the equilibrium must be the same. We therefore have invariance with respect to changes in parameters as long as $(\alpha+\beta) / t$ remains constant. 


\section{Appendix B: Existence of a spatial equilibrium}

\section{Formulation of a fixed point}

We denote the set of continuous functions on $\mathcal{B}=[-b, b]$ by $C^{0}(\mathcal{B})$ and the set of functions with continuous first derivative by $C^{1}(\mathcal{B})$. To formulate the fixed point problem, we work with the commuting flow function $n$ which is continuous: that is, $n \in \mathcal{N}$ where $\mathcal{N} \equiv\left\{n \in C^{0}(\mathcal{B}): n(-b)=n(b)=0\right.$, $\dot{n} \in[-1 / s, 1]$ a.e. $\}$ where a.e. means almost everywhere, that is everywhere except on a zero measure set. We also use a concise notation for operators where $M n$ expresses the application of operator $M: \mathcal{N} \rightarrow C^{0}(\mathcal{B})$ on a function $n \in \mathcal{N}$ and $M n(z)$ expresses the value returned by $M n$ at $z \in \mathcal{B}$.

We redefine the economic surplus as the operator $S: \mathcal{N} \rightarrow C^{1}(\mathcal{B})$ and its slope $\dot{S}: \mathcal{N} \rightarrow C^{0}(\mathcal{B})$ so that $S n(z)=S n(-b)+\int_{\mathcal{B}} \dot{S} n(x) \cdot 1_{\{z>x\}} d x$ where $1_{\{A\}}$ is the indicator function that returns 1 if $A$ is true and 0 otherwise. Then, we denote the rent differential by $\Delta \Psi: \mathcal{N} \rightarrow C^{0}(\mathcal{B}),(\Delta \Psi)(z)=$ $\int_{\mathcal{B}}\left(\dot{\Psi}_{b}-\dot{\Psi}_{r}\right) \cdot 1_{\{z>x\}} \mathrm{d} x$. Using the value for $\dot{\Psi}_{b}-\dot{\Psi}_{r}$ in (54) and (55), integrating from $z=-b$, and using rent differential (53) at this location, we get

$$
\Delta \Psi n(z)=\frac{1}{s}\left(\alpha \ln \frac{\alpha \rho}{\gamma \mathrm{e}}+\beta \ln \frac{\beta \rho}{\gamma \mathrm{e}}+\bar{c}_{0}-U^{*}\right)+\frac{1}{s}(\alpha+\beta) \operatorname{Sn}(z)-\frac{1+s}{s} t \int_{-b}^{z} \operatorname{sign}(n(x)) \mathrm{d} x
$$

We rewrite the bid rent differential in a more compact form as

$$
\Delta \Psi=\frac{1}{s}\left(\Lambda+\Lambda_{0}-U\right)
$$

where the operator $\Lambda: \mathcal{N} \rightarrow C^{0}(\mathcal{B})$ is defined by $\Lambda=(\alpha+\beta) S-(1+s) t L$, the operator $L: \mathcal{N} \rightarrow$ $C^{0}(\mathcal{B})$ by $\operatorname{Ln}(z)=\int_{\mathcal{B}} \operatorname{sign}(n(x)) \cdot 1_{\{z>x\}} \mathrm{d} x$, and the scalar $\Lambda_{0}$ by $s\left(\alpha \ln \frac{\alpha \rho}{\gamma \mathrm{e}}+\beta \ln \frac{\beta \rho}{\gamma \mathrm{e}}+\bar{c}_{0}\right)$.

A spatial equilibrium is then defined by the function $n:[-b, b] \rightarrow \mathbb{R}$ and the scalar $U$ which are the solutions to

$$
\dot{n} \in\left\{\begin{array}{ccc}
\{1\} & \text { if } & \Lambda n+\Lambda_{0}-U<0 \\
{\left[-\frac{1}{s}, 1\right]} & \text { if } & \Lambda n+\Lambda_{0}-U=0 \\
\left\{-\frac{1}{s}\right\} & \text { if } & \Lambda n+\Lambda_{0}-U>0
\end{array}\right.
$$

with $\dot{n} \geq 0=n$ at $z= \pm b$.

We now formulate the fixed point problem in terms of the continuous function $n$ rather than the discontinuous function $\dot{n}$. Towards this aim, we integrate the previous condition (56). To take care of the three subconditions, we define the correspondence $F: \mathbb{R} \rightarrow[-1 / s, 1]$ such that $F(z)=-1 / s$ if 
$z<0, F(z)=[-1 / s, 1]$ if $z=0$, and $F(z)=1$ if $z>0$. We define the functional $U^{*}: \mathcal{N} \rightarrow \mathbb{R}$ that for any function $n$, gives the scalar $U$ that solves

$$
0=\int_{\mathcal{B}} F\left[U-\Lambda_{0}-\Lambda n(z)\right] \mathrm{d} z
$$

and the operator $O: \mathcal{N} \rightarrow \mathcal{N}^{\prime}$

$$
(O n)(z)=\int_{\mathcal{B}} F\left[U^{*} n-\Lambda_{0}-\Lambda n(x)\right] \cdot 1_{\{z>x\}} \mathrm{d} x
$$

The definition of $U^{*}$ guarantees that $n(b)=0$ while $n(-b)=0$ is trivially satisfied. By construction, the operator $O$ returns a function $n$ such that $\dot{n} \in[-1 / s, 1]$. So, $O$ maps $\mathcal{N}$ into itself.

Lemma 5 The existence of a spatial equilibrium is equivalent to the existence of a fixed point $n \in$ On.

\section{Sequences of fixed points}

The Schauder theorem applies on a set of continuous mappings of continuous functions. So, we use continuous approximations of $F$ and sign functions and then prove convergence. We define the continuous, differentiable and strictly increasing functions $F_{k}: \mathbb{R} \rightarrow[-1 / s, 1], k \in \mathbb{N}$, such that $F_{k}$ converges to $F$ as $k \rightarrow \infty$ and $0<F_{k}^{\prime} \leq k$, and the continuous, differentiable and strictly increasing function $H_{l}: \mathbb{R} \rightarrow[-1,1], l \in \mathbb{N}$, such that $H_{l}$ converges to sign as $l \rightarrow \infty$ and $0<H_{l}^{\prime} \leq l$. In particular, we consider logistic functions with images on $[-1 / s, 1]$ and $[-1,1]: F_{k}(z)=-1+$ $(1+s) /\left[1+\exp (-4 k z /(s+1)]\right.$ and $H_{l}(z)=-1+2 /[1+\exp (-2 l z)]$. The type of convergence will be defined formally below.

We use the subset of continuous functions $\mathcal{N}$ and the $\sup$ norm $\|n\|_{\infty}=\sup _{z \in \mathcal{B}}|n(z)|$. We define the operators $\Lambda_{l}$ and $L_{l}: \mathcal{N} \rightarrow \mathcal{C}^{0}(\mathcal{B})$ by

$$
\begin{aligned}
& \Lambda_{l} n(z)=(\alpha+\beta) S n(z)-(1+s) t L_{l} n(z) \\
& L_{l} n(z)=\int_{\mathcal{B}} H_{l}(n(x)) \cdot 1_{\{z>x\}} \mathrm{d} x
\end{aligned}
$$

Furthermore we define the functional $U_{k l}^{*}: \mathcal{N} \rightarrow \mathbb{R}$ as being the solution $U$ to $\int_{\mathcal{B}} F_{k}\left[U-\Lambda_{0}-\right.$ $\left.\Lambda_{l} n(x)\right] \mathrm{d} x=0$ and the operator $O_{k l}: \mathcal{N} \rightarrow \mathcal{N}$ by

$$
O_{k l} n(z)=\int_{\mathcal{B}} F_{k}\left[U_{k l}^{*} n-\Lambda_{0}-\Lambda_{l} n(x)\right] \cdot 1_{\{z>x\}} \mathrm{d} x
$$


Note that $U_{k l}^{*}$ is a unique solution because $F_{k}$ is strictly increasing.

Our strategy is to prove the existence of fixed points $n_{k l}^{*}$ to $n_{k l}^{*}=O_{k l} n_{k l}^{*} \forall k, l \in \mathbb{N}$ and prove that the limit $n^{*}=\lim _{k \rightarrow \infty} \lim _{l \rightarrow \infty} n_{k l}^{*}$ satisfies $n^{*}=O n^{*}$. We first prove that the set $\mathcal{N}$ is a closed convex set in the Banach space $C^{0}(\mathcal{B})$ and the mapping $O_{k l}$ is well defined and continuous.

Lemma 6 The set $\mathcal{N}$ is a closed convex set in the Banach space $C^{0}(\mathcal{B})$.

Proof. The space $C^{0}(\mathcal{B})$ is a vector space of continuous scalar functions on the real compact interval $\mathcal{B}=[-b, b]$ for the operation of addition and scalar multiplication. It is equipped with the sup norm $\|n\|=\sup _{z \in \mathcal{B}}|n|$. Every Cauchy sequence $\left\{n_{k}\right\}_{k=1}^{\infty}$ with $n_{k} \in \mathcal{N}$ converges to some function $n \in \mathcal{N}$. It is thus a Banach space. The set of continuous functions $\mathcal{N}=\left\{n \in C^{0}(\mathcal{B}): n(-b)=n(b)=\right.$ $0, \dot{n} \in[-1 / s, 1]\}$ is closed and convex. Indeed, any convex combination $\alpha n+(1-\alpha) m, n, m \in \mathcal{N}$, belongs to $\mathcal{N}$. It is also closed because every sequence $\left\{n_{k}\right\}_{k=1}^{\infty}$ with $n_{k} \in \mathcal{N}$ converges to some $n \in \mathcal{N}$.

We now check that $O_{k l}$ is a well-defined continuous mapping. We remind that an operator is well defined if it actually maps its domain into the defined set of images. An operator $M: X \rightarrow Y$, with $X, Y \in C^{0}(\mathcal{B})$, is a continuous mapping if for all $\delta>0$, there exists $k>0$ such that for any $n, m \in X$, $\|n-m\|_{\infty}<1 / k \Rightarrow\|M n-M m\|_{\infty}<\delta$. It must be noted that the composition of continuous mappings is also a continuous mapping. Also, the convolution $M: X \rightarrow Y, M n(z)=\int_{\mathcal{B}} f(z-x) n(x) \mathrm{d} x$ is a continuous mapping for any bounded valued function $f: \mathbb{R} \rightarrow \mathbb{R},|f|<\infty$.

Lemma 7 S is a well defined continuous mapping.

Proof. We first prove the following useful statement: An operator $M: \mathcal{N} \rightarrow C^{0}(\mathbb{R}), M n(z)=$ $\int_{\mathcal{B}} f(x, n) g(x-z) \dot{n}(x) \mathrm{d} x$ is a continuous mapping when we consider $f: \mathcal{B} \times \mathcal{N} \rightarrow \mathbb{R}$ as a continuous function in $m$ with bounded image (i.e. $|f|<f^{\max }$ ) and $g: \mathbb{R} \rightarrow \mathbb{R}^{+}$with bounded image (i.e. $|g|<$ 
$\left.g^{\max }\right)$. We indeed successively get

$$
\begin{aligned}
\|M n-M m\|_{\infty} & =\sup _{z \in \mathcal{B}}\left|\int_{\mathcal{B}} f(x, n) g(x-z) \dot{n}(x) \mathrm{d} x-\int_{\mathcal{B}} f(x, m) g(x-z) \dot{m}(x) \mathrm{d} x\right| \\
& =\sup _{z \in \mathcal{B}}\left|\begin{array}{c}
\int_{\mathcal{B}} f(x, n) g(x-z) \dot{n}(x) \mathrm{d} x-\int_{\mathcal{B}} f(x, m) g(x-z) \dot{m}(x) \mathrm{d} x \\
-\int_{\mathcal{B}} f(x, n) g(x-z) \dot{m}(x) \mathrm{d} x+\int_{\mathcal{B}} f(x, n) g(x-z) \dot{m}(x) \mathrm{d} x
\end{array}\right| \\
& \leq \sup _{z \in \mathcal{B}}\left|\int_{\mathcal{B}} f(x, n) g(x-z)(\dot{n}(x)-\dot{m}(x)) \mathrm{d} x\right|+\left|\int_{\mathcal{B}}[f(x, m)-f(x, n)] g(x-z) \dot{m}(x) \mathrm{d} x\right| \\
& \leq f^{\max } g^{\max }\left|\int_{\mathcal{B}}(\dot{n}(x)-\dot{m}(x)) \mathrm{d} x\right|+g^{\max }\left|\int_{\mathcal{B}}[f(x, m)-f(x, n)] \dot{m}(x) \mathrm{d} x\right|
\end{aligned}
$$

Note that $\int_{\mathcal{B}}(\dot{n}(x)-\dot{m}(x)) \mathrm{d} x=\int_{-b}^{b} \mathrm{~d} n(x)-\int_{-b}^{b} \mathrm{~d} m(x)=n(b)-n(-b)-[m(b)-m(-b)]=0$ since $n( \pm b)=m( \pm b)=0$ for any $n, m \in \mathcal{N}$. Also, for any $m \in \mathcal{N}$, one has that $\dot{m} \in[-1 / s, 1]$ and therefore $|\dot{m}| \leq \max \{1,1 / s\}$. Using this,

$$
\begin{aligned}
\sup _{z \in \mathcal{B}}\left|\int_{\mathcal{B}}[f(x, m)-f(x, n)] \dot{m}(x) \mathrm{d} x\right| & \leq \max \left\{1, \frac{1}{s}\right\} \cdot\left|\int_{\mathcal{B}}[f(x, m)-f(x, n)] \mathrm{d} x\right| \\
& \leq \max \left\{1, \frac{1}{s}\right\} \cdot 2 b \sup _{z \in \mathcal{B}}|f(z, m)-f(z, n)|
\end{aligned}
$$

Finally, by continuity of $f$, for any $\delta>0$, there exists $\epsilon>0$ such that $\|m-n\|<\epsilon$ implies $|f(z, m)-f(z, n)|<\delta$ for all $z$, or equivalently, $\sup _{z \in \mathcal{B}}|f(z, m)-f(z, n)|<\delta$. Thus, for any $\delta^{\prime}$ we can use the same $\epsilon$ such that $\|m-n\|<\epsilon \Rightarrow\|M n-M m\|_{\infty}<\delta^{\prime}$ where we set $\delta=\delta^{\prime} /\left[2 b g^{\max } \max \{1,1 / s\}\right]$. This proves the continuity of $M$.

Second, the operator $T^{1-\sigma}: \mathcal{N} \rightarrow C^{0}(\mathbb{R})$ with $T^{1-\sigma} n(z)=\int_{\mathcal{B}} e^{(1-\sigma) \tau|z-x|} \mu(x) \mathrm{d} x$ is a well-defined continuous operator. Indeed, using $\mu=(1-\dot{n}) /(1+s)$, we have $T^{1-\sigma} n(z)=\int_{\mathcal{B}} e^{(1-\sigma) \tau|z-x|} \mathrm{d} x /(1+$ $s)-\int_{\mathcal{B}} e^{(1-\sigma) \tau|z-x|} \dot{n} \mathrm{~d} x /(1+s)$. The first term is independent of $n$. Regarding the second term, just set $f(x, n)=1$ and $g(z-x)=e^{(1-\sigma) \tau|z-x|}$, which have bounded images for $x, z \in \mathcal{B}$. The operator is well defined as it returns continuous functions having images in a strictly positive range: $T^{1-\sigma} n(\mathcal{B}) \subset\left(2 b e^{(1-\sigma) 2 b \tau} /(1+s), 2 b / s\right)$. For the highest bound, we simply plug $\dot{n}=-1 / s$ in the first line of the above expression. For the lowest bound, we compute $T^{1-\sigma} n(y)=\int_{\mathcal{B}} e^{(1-\sigma) \tau|z-y|} \frac{1-\dot{n}(z)}{1+s} \mathrm{~d} z \geq$ $e^{(1-\sigma) 2 b \tau} \int_{\mathcal{B}} \frac{1-\dot{n}(z)}{1+s} \mathrm{~d} z=\frac{2 b e^{(1-\sigma) 2 b \tau}}{1+s}>0$ where we use $|z-y|<2 b$ in the inequality and $\int_{\mathcal{B}} \dot{n} \mathrm{~d} z=0$ in the last equality. Therefore, $1 /\left[\sigma T^{1-\sigma} n(x)\right]$ is also a continuous function having images in a strictly positive range while $\ln \left[T^{1-\sigma} n(x)\right]$ is a continuous functions having real bounded images.

Third, the operator $G: \mathcal{N} \rightarrow C^{0}(\mathbb{R})$ with $G n(z)=\frac{1}{\sigma} \int_{\mathcal{B}} \frac{e^{-\sigma \tau|z-x|}}{\left(T^{1-\sigma} n\right)(x)} \mu(x) \mathrm{d} x$ is a well-defined continuous operator. Indeed, one just has to set $f(x, n)=1 /\left[\sigma T^{1-\sigma} n(x)\right]$ and $g(z-x)=e^{-\sigma \tau|z-x|}$ in the 
above statement. It is clear that the operator returns a function with bounded positive images.

Finally, since $S=G-\ln T=G+\frac{1}{\sigma-1} \ln T^{1-\sigma}$, it is a linear combination of well defined continuous operators. Thus, we can conclude that $S: \mathcal{N} \rightarrow C^{0}(\mathcal{B})$ is a well-defined continuous mapping.

Lemma $8 L_{l}$ is a well-defined continuous mapping.

Proof. For any $\delta>0$, we must find $\epsilon$ such that $\|m-n\|_{\infty}<\epsilon \Rightarrow\left\|L_{l} n-L_{l} m\right\|_{\infty}<\delta$. We successively get

$$
\begin{aligned}
\left\|L_{l} n-L_{l} m\right\| & =\sup _{z \in \mathcal{B}}\left|\int_{\mathcal{B}} H_{l}(n(x)) \cdot 1(z>x) \mathrm{d} x-\int_{\mathcal{B}} H_{l}(m(x)) \cdot 1_{\{z>x\}} \mathrm{d} x\right| \\
& =\sup _{z \in \mathcal{B}}\left|\int_{\mathcal{B}}\left[H_{l}(n(x))-H_{l}(m(x))\right] \cdot 1_{\{z>x\}} \mathrm{d} x\right| \\
& =\sup _{z \in \mathcal{B}}\left|\int_{\mathcal{B}}[n(x)-m(x)] H_{l}^{\prime}(p(x)) \cdot 1_{\{z>x\}} \mathrm{d} x\right|
\end{aligned}
$$

where $p(x) \in[\min (n(x), m(x)), \max (n(x), m(x))]$ by the mean value theorem and the continuity and the differentiability of $H_{l}$. Using the fact that $H_{l}^{\prime} \leq l$ and that $n(-b)=m(-b)=0$, we have

$$
\begin{aligned}
\left\|L_{l} n-L_{l} m\right\|_{\infty} & \leq l \sup _{z \in \mathcal{B}}\left|\int_{-b}^{z}[n(x)-m(x)] \mathrm{d} x\right| \\
& \leq l \sup _{z \in \mathcal{B}} \int_{-b}^{z}|n(x)-m(x)| \mathrm{d} x \\
& =2 b l \sup _{z \in \mathcal{B}}|n(z)-m(z)| \\
& =2 b l\|n-m\|
\end{aligned}
$$

So, for any $\delta$, choose $\epsilon<\delta / l$. Therefore, $L_{l}: \mathcal{N} \rightarrow C^{0}(\mathcal{B})$ is a continuous mapping. Since $H_{l}$ has image on $[-1,1]$, the image of $L_{l}$ lies in the interval $[-2 b, 2 b]$. So, it is well defined.

Since $\Lambda_{l}: \mathcal{N} \rightarrow C^{0}(\mathcal{B})$ is composition of well-defined continuous mappings with bounded images, it is also a well defined continuous mapping. We finally need to show the corresponding result for $O_{k l}$ which depends on $U_{k l}^{*}$.

Lemma $9 U_{k l}^{*}$ is a well-defined continuous functional.

Proof. For readability we here temporarily write $U_{k l}^{*}$ as $U$. Then, $U: \mathcal{N} \rightarrow \mathbb{R}$ is the unique solution to $\int_{\mathcal{B}} F_{k}\left[U-\Lambda_{0}-\Lambda_{l} n(x)\right] \mathrm{d} x=0$ because $F_{k}$ is strictly increasing. Also, it is clear that the 
solution $U$ must lie between $\min _{x \in \mathcal{B}}\left[\Lambda_{0}+\Lambda_{l} n(x)\right]$ and $\max _{x \in \mathcal{B}}\left[\Lambda_{0}+\Lambda_{l} n(x)\right]$. Since $\Lambda_{l} n$ has bounded image, $U n$ has also bounded images and is well defined.

Let us consider two commuting functions $n$ and $m: \mathcal{N} \rightarrow \mathbb{R}$. Let also $p^{\text {min }}$ and $p^{\max }: \mathcal{N} \times \mathcal{N} \rightarrow \mathbb{R}$ be the minimum and the maximum functions of $U n-\Lambda_{l} n$ and $U m-\Lambda_{l} m$. By the mean value theorem and the differentiability of $F_{k}$, we have

$$
F_{k}\left[U n-\Lambda_{0}-\Lambda_{l} n(x)\right]-F_{k}\left[U m-\Lambda_{0}-\Lambda_{l} m(x)\right]=F_{k}^{\prime}(p(x)) \cdot\left[U n-\Lambda_{l} n(x)-U m+\Lambda_{l} m(x)\right]
$$

where $p: \mathcal{B} \rightarrow \mathbb{R}$ such that $p \in\left[p^{\min }, p^{\max }\right]$. This yields

$$
\begin{aligned}
F_{k}^{\prime}(p(x)) \cdot\left(U n-U_{k l} m\right) & =F_{k}\left[U n-\Lambda_{0}-\Lambda_{l} n(x)\right]-F_{k}\left[U m-\Lambda_{0}-\Lambda_{l} m(x)\right] \\
& +F_{k}^{\prime}(p(x)) \cdot\left[\Lambda_{l} n(x)-\Lambda_{l} m(x)\right]
\end{aligned}
$$

Integrating, applying $\int_{\mathcal{B}} F_{k}\left[U-\Lambda_{0}-\Lambda_{l} n(x)\right] \mathrm{d} x=0$ to $n$ and $m$, and subtracting expressions, we get

$$
(U n-U m) \cdot \int_{\mathcal{B}} F_{k}^{\prime}(p(x)) \mathrm{d} x=\int_{\mathcal{B}} F_{k}^{\prime}(p(x)) \cdot\left[\Lambda_{l} n(x)-\Lambda_{l} m(x)\right] \mathrm{d} x
$$

Since $F_{k}^{\prime}>0$, we have

$$
\begin{aligned}
|U n-U m| & =\frac{\int_{\mathcal{B}} F_{k}^{\prime}(p(x)) \cdot\left[\Lambda_{l} n(x)-\Lambda_{l} m(x)\right] \mathrm{d} x}{\int_{\mathcal{B}} F_{k}^{\prime}(p(x)) \mathrm{d} x} \\
& \leq \frac{\int_{\mathcal{B}} F_{k}^{\prime}(p(x)) \cdot\left|\Lambda_{l} n(x)-\Lambda_{l} m(x)\right| \mathrm{d} x}{\int_{\mathcal{B}} F_{k}^{\prime}(p(x)) \mathrm{d} x} \\
& \leq \frac{\int_{\mathcal{B}} F_{k}^{\prime}(p(x)) \cdot \sup _{z}\left|\Lambda_{l} n(z)-\Lambda_{l} m(z)\right| \mathrm{d} x}{\int_{\mathcal{B}} F_{k}^{\prime}(p(x)) \mathrm{d} x} \\
& =\sup _{z}\left|\Lambda_{l} n(z)-\Lambda_{l} m(z)\right| \\
& =\left\|\Lambda_{l} n-\Lambda_{l} m\right\|_{\infty}
\end{aligned}
$$

In other words $U n$ is a continuous mapping of $\Lambda_{l} n$. Since $\Lambda_{l} n$ is a continuous mapping of $n, U n$ is also a continuous mapping of $n$.

Lemma 10 For any $k, l \in \mathbb{N}, O_{k l}: \mathcal{N} \rightarrow \mathcal{N}$ is a continuous mapping.

Proof. The operator $O_{k l}$ is defined by

$$
O_{k l} n(z)=\int_{\mathcal{B}} F_{k}\left[U_{k l}^{*} n-\Lambda_{l} n(x)\right] \cdot 1_{\{z>x\}} \mathrm{d} x
$$


where $U_{k l}^{*} n$ was discussed in the previous lemma. By construction, the image functions $m \in O_{k l} n$ are continuous and satisfy $m(-b)=m(b)=0$ and $\dot{m} \in[-1 / s, 1]$. The operator $O_{k l}$ is well defined as it is a mapping $\mathcal{N} \rightarrow \mathcal{N}$.

For readability we temporarily dispense $U_{k l}^{*}$ and $O_{k l}$ with the subscripts $k l$ and superscript $*$. Using the mean value theorem and the differentiability of $F_{k}$ and $p$ defined in the previous lemma, we get

$$
\begin{aligned}
\|O n-O m\|_{\infty} & =\sup _{z \in \mathcal{B}}\left|\int_{\mathcal{B}}\left\{F_{k}\left[U n-\Lambda_{l} n(x)\right]-F_{k}\left[U n-\Lambda_{l} n(x)\right]\right\} \cdot 1_{\{z>x\}} \mathrm{d} x\right| \\
& =\sup _{z \in \mathcal{B}}\left|\int_{\mathcal{B}} F_{k}^{\prime}(p(x)) \cdot\left[U n-\Lambda_{l} n(x)-U(m)+\Lambda_{l} m(x)\right] \cdot 1_{\{z>x\}} \mathrm{d} x\right| \\
& \leq \sup _{z \in \mathcal{B}}\left|\int_{\mathcal{B}} F_{k}^{\prime}(p(x)) \cdot\left[\Lambda_{l} n(x)-\Lambda_{l} m(x)\right] \cdot 1_{\{z>x\}} \mathrm{d} x\right| \\
& +|U n-U m| \cdot \sup _{z \in \mathcal{B}}\left|\int_{\mathcal{B}} F_{k}^{\prime}(p(x)) \cdot 1_{\{z>x\}} \mathrm{d} x\right|
\end{aligned}
$$

We successively have

$$
\begin{aligned}
\sup _{z \in \mathcal{B}} \int_{\mathcal{B}}\left|F_{k}^{\prime}(p(x))\left[\Lambda_{l} n(x)-\Lambda_{l} m(x)\right]\right| \cdot 1(x<z) \mathrm{d} x & \leq \sup _{z \in \mathcal{B}} \int_{\mathcal{B}}\left|F_{k}^{\prime}(p(x))\right| \cdot\left|\Lambda_{l} n(x)-\Lambda_{l} m(x)\right| \cdot 1_{\{z>x\}} \mathrm{d} x \\
& \leq \int_{\mathcal{B}}\left|F_{k}^{\prime}(p(x))\right| \cdot\left|\Lambda_{l} n(x)-\Lambda_{l} m(x)\right| \mathrm{d} x \\
& \leq k \int_{\mathcal{B}}\left|\Lambda_{l} n(x)-\Lambda_{l} m(x)\right| \mathrm{d} x \\
& \leq 2 b k \sup _{z \in \mathcal{B}}\left|\Lambda_{l} n(z)-\Lambda_{l} m(z)\right| \\
& =2 b k\left\|\Lambda_{l} n-\Lambda_{l} m\right\|_{\infty}
\end{aligned}
$$

and

$$
\begin{aligned}
|U n-U m| \cdot \sup _{z \in \mathcal{B}}\left|\int_{\mathcal{B}} F_{k}^{\prime}(p(x)) \cdot 1(x<z) \mathrm{d} x\right| & \leq\left\|\Lambda_{l} n-\Lambda_{l} m\right\|_{\infty} \cdot \sup _{z \in \mathcal{B}} \int_{\mathcal{B}}\left|F_{k}^{\prime}(p(x))\right| 1(x<z) \mathrm{d} x \\
& \leq\left\|\Lambda_{l} n-\Lambda_{l} m\right\|_{\infty} \cdot \int_{\mathcal{B}}\left|F_{k}^{\prime}(p(x))\right| \mathrm{d} x \\
& \leq 2 b k\left\|\Lambda_{l} n-\Lambda_{l} m\right\|
\end{aligned}
$$

Finally,

$$
\|O n-O m\|_{\infty} \leq 4 b k\left\|\Lambda_{l} n-\Lambda_{l} m\right\|_{\infty}
$$

For any $\delta$, we can then choose $\epsilon=\delta /(4 b k)$ so that $\left\|\Lambda_{l} n-\Lambda_{l} m\right\|_{\infty}<\epsilon$ implies $\|O n-O m\|_{\infty}<\delta$. Hence, putting back the subscripts $k l, O_{k l}$ is a continuous mapping of $\Lambda_{l}$. Since $\Lambda_{l}$ is a continuous mapping of $n$, we have that $O_{k l}$ is also a continuous mapping of $n$. 
Lemma 11 The image $O_{k l}(\mathcal{N})$ is a relatively compact subset of $\mathcal{N}$.

Proof. By Arzela-Ascoli theorem, this is true if $n \in O_{k l}(\mathcal{N})$ are uniformly bounded and equicontinuous on $\mathcal{B}$. To say that $n \in O_{k l}(\mathcal{N})$ are uniformly bounded means that there exists $M>0$ such that $\|n\|_{\infty}=\sup _{z \in \mathcal{B}}|n(z)|<M$ for all $n \in O_{k l}(\mathcal{N})$. This is true since $F_{k}$ has image on $[-1 / s, 1]$. Therefore, $O_{k l}$ generates functions $n: \mathcal{B} \rightarrow \mathbb{R}$ such that $n(-b)=n(b)=0$ and $\dot{n} \in[-1 / s, 1]$. These functions have image in $[-2 n, 2 b]$. To say that $n$ is equicontinuous on $\mathcal{B}$ means that for every $\delta>0$ there exists an $\epsilon$ such that for every $x, y \in \mathcal{B}$ and every $n \in \mathcal{N}$, we have $|x-y|<\epsilon \Rightarrow|n(x)-n(y)|<\delta$. This follows from the continuity of $n \in C^{0}(\mathcal{B})$.

To sum up, the set $\mathcal{N}$ is a closed convex set in the Banach space $C^{0}(\mathcal{B})$. The operator $O_{k l}: \mathcal{N} \rightarrow \mathcal{N}$ is a continuous mapping such that $O_{k l}(\mathcal{N})$ is a relatively compact subset of $\mathcal{N}$. We can then apply Schauder fixed point theorem.

Lemma 12 For each $k, l \in \mathbb{N}$, there exists a fixed point $n_{k l}^{*} \in \mathcal{N}$ such that $n_{k l}^{*}=O_{k l} n_{k l}^{*}$.

\section{Convergence}

We now turn to the question about whether the sequence of fixed points $\left\{n_{k l}^{*}\right\}_{k, l \in \mathbb{N}}$ converges to a function that is also a fixed point of (57). Because $\mathcal{N}$ is relatively compact and any $n_{k l}^{*}$ belongs to $\mathcal{N}$, the sequence $\left\{n_{k l}^{*}\right\}_{k, l \in \mathbb{N}}$ converges uniformly on $\mathcal{B}$ to a function $n_{\infty}^{*} \in \mathcal{N}$.

Note that $F$ is a correspondence because $F(0)=[-1 / s, 1]$. In turn, the operator $O: \mathcal{N} \rightarrow \mathcal{N}$ is a correspondence. We therefore want to check whether $n_{\infty}^{*} \in O n_{\infty}^{*}$. Formally, we ask whether for any $\delta>0$, there exists a function $n^{*} \in O n_{\infty}^{*}$ and two positive integers $\bar{k}$ and $\bar{l}$ such that we have

$$
\left\|O_{k l} n_{\infty}^{*}-n^{*}\right\|_{\infty}<\delta
$$

for $k>\bar{k}$ and $l>\bar{l}$.

We here assume that the sequence $\left\{H_{l}\right\}_{l \in \mathbb{N}}$ converges to the sign function under the norm $\|\cdot\|_{1}$. Formally, for any $\delta>0$, there exists $\bar{l} \in \mathbb{N}$ such that $\int\left|H_{l}(\xi)-\operatorname{sign}(\xi)\right| \mathrm{d} \xi<\delta$. Similarly, we assume that $\left\{F_{k}\right\}_{k \in \mathbb{N}}$ converges to $F$ under the norm $\|\cdot\|_{1}$. That is, for any $\delta>0$, there exists $\bar{k} \in \mathbb{N}$ such that $\int\left|F_{k}(\xi)-F(\xi)\right| \mathrm{d} \xi<\delta$. The last integral returns a scalar because the correspondence $F$ returns a set only on the zero measure set at $\xi=0$.

Lemma $13 \Lambda_{l}$ converges to $\Lambda$ uniformly under the norm $\|\cdot\|_{\infty}$. 
Proof. The mapping $\Lambda_{l}$ uniformly converges to the mapping $\Lambda$ if for any $\delta>0$, we can find $\bar{l}$ such that $\left\|\Lambda_{l}-\Lambda\right\|_{\infty} \leq \delta$ for all $l>\bar{l}$. Note that $\left\|\Lambda_{l}-\Lambda\right\|_{\infty}=\left\|(\alpha+\beta) S-(1+s) t L_{l}-(\alpha+\beta) S+(1+s) t L\right\|_{\infty}$ $=(1+s) t\left\|L_{l}-L\right\|_{\infty}$. So, we just need to check the uniform convergence of the mapping $L_{l}: \mathcal{N} \rightarrow \mathbb{R}$, $L_{l} n(z)=\int_{\mathcal{B}} H_{l}(n(x)) \cdot 1_{\{z>x\}} \mathrm{d} x$ to the mapping $\operatorname{Ln}(z)=\int_{\mathcal{B}} \operatorname{sign}(n(x)) \cdot 1_{\{z>x\}} \mathrm{d} x$. By the definition of the convergence for $H_{l}$, for any $\delta>0$, we can find $\bar{l}$ such that $\int\left|H_{l}(\xi)-\operatorname{sign}(\xi)\right| \mathrm{d} \xi \leq \delta$ for all $l>\bar{l}$. Furthermore we have

$$
\begin{aligned}
\left\|L_{l}-L\right\|_{\infty} & =\sup _{z \in \mathcal{B}}\left|\int_{\mathcal{B}}\left[H_{l}(n(x))-\operatorname{sign}(n(x))\right] \cdot 1_{\{z>x\}} \mathrm{d} x\right| \\
& \leq \int_{\mathcal{B}}\left|H_{l}(n(x))-\operatorname{sign}(n(x))\right| \mathrm{d} x \\
& =\int\left|H_{l}(\xi)-\operatorname{sign}(\xi)\right| \cdot \mathcal{M}(x: \xi \leq n(x)<\xi+\mathrm{d} \xi) \mathrm{d} \xi \\
& \leq 2 b \int\left|H_{l}(\xi)-\operatorname{sign}(\xi)\right| \mathrm{d} \xi \\
& \leq 2 b \delta
\end{aligned}
$$

where $\mathcal{M}(\mathcal{E})$ denotes the measure of the set $\mathcal{E} \subseteq \mathcal{B}$ so that $\mathcal{M}(\mathcal{B})=2 b$. So, for any $\delta^{\prime}$, we can use the above integer $\bar{l}$ for $\delta=\delta^{\prime} / 2 b$ and get $\left\|L_{l}-L\right\|_{\infty}<\delta^{\prime}$ for all $l>\bar{l}$. Therefore, $L_{l}$ converges uniformly to $L$. The same conclusion applies to $\Lambda_{l}$ and $\Lambda$. That is, the sequence $\left\{\Lambda_{l} n\right\}_{l \in \mathbb{N}}$ converges to $\Lambda n$.

Let the function $\widehat{F}: \mathbb{R} \rightarrow[-1 / s, 1]$ be such that $\widehat{F}(\xi)=1$ if $\xi>0, \widehat{F}(\xi)=0$ if $\xi=0$, and $\widehat{F}(\xi)=-1 / s$ if $\xi<0$. It is clear that $\widehat{F} \in F$ and that $\left\{F_{k}\right\}_{k \in \mathbb{N}}$ converges to $\widehat{F}$ under the norm $\|\cdot\|_{1}$. For any $\delta>0$, there indeed exists $\bar{k} \in \mathbb{N}$ such that $\int_{-\infty}^{\infty}\left|F_{k}(\xi)-\widehat{F}(\xi)\right| d \xi<\delta$. This is true because $\left\{F_{k}\right\}_{k \in \mathbb{N}}$ converges to $F$ and $\int_{-\infty}^{\infty}\left|F_{k}(\xi)-\widehat{F}(\xi)\right| d \xi=\int_{-\infty}^{\infty}\left|F_{k}(\xi)-F(\xi)\right| d \xi$ since $F$ and $\widehat{F}$ differ only on a zero measure set.

Lemma 14 For any operator $Z: \mathcal{N} \rightarrow C^{0}(\mathcal{B})$ and function $n \in \mathcal{N}, \int_{\mathcal{B}} F_{k}[Z n(x)] \cdot 1_{\{z>x\}} \mathrm{d} x$ converges uniformly to $\int_{\mathcal{B}} \widehat{F}[Z n(x)] \cdot 1_{\{z>x\}} \mathrm{d} x$ under the norm $\|\cdot\|_{\infty}$ and $\int_{\mathcal{B}} \widehat{F}[Z n(x)] \cdot 1_{\{z>x\}} \mathrm{d} x \in \int_{\mathcal{B}} F[Z n(x)]$. $1_{\{z>x\}} \mathrm{d} x$.

Proof. We first prove that for any $\delta>0$ there exists a positive integer $\bar{k} \in \mathbb{N}$ such that

$$
I_{k}=\left\|\int_{\mathcal{B}} \widehat{F}(Z n(x)) \cdot 1_{\{z>x\}} \mathrm{d} x-\int_{\mathcal{B}} F_{k}[Z n(x)] \cdot 1_{\{z>x\}} \mathrm{d} x\right\|_{\infty}<\delta
$$


for $k>\bar{k}$. We indeed successively have

$$
\begin{aligned}
I_{k} & =\left\|\int_{\mathcal{B}}\left\{\widehat{F}(Z n(x))-F_{k}[Z n(x)]\right\} \cdot 1_{\{z>x\}} \mathrm{d} x\right\|_{\infty} \\
& =\sup _{z \in \mathcal{B}}\left|\int_{\mathcal{B}}\left\{\widehat{F}(Z n(x))-F_{k}[Z n(x)]\right\} \cdot 1_{\{z>x\}} \mathrm{d} x\right| \\
& \leq \int_{\mathcal{B}}\left|\widehat{F}(Z n(x))-F_{k}[Z n(x)]\right| \mathrm{d} x \\
& =\int\left|\widehat{F}(\xi)-F_{k}(\xi)\right| \cdot \mathcal{M}_{\{x: \xi \leq Z n(x)<\xi+\mathrm{d} \xi\}} \mathrm{d} \xi
\end{aligned}
$$

where $\mathcal{M}_{\mathcal{E}}$ denotes the measure of a set $\mathcal{E} \subseteq \mathcal{B}$ so that $\mathcal{M}_{\mathcal{B}}=2 b$. We can use the fact that the measure is always smaller than $2 b$ and get

$$
I_{k} \leq 2 b \int\left|\widehat{F}(\xi)-F_{k}(\xi)\right| \mathrm{d} \xi
$$

Finally, using the definition of convergence of $F_{k}$ to $\widehat{F}$, we can find a positive integer $\bar{k}$ such that $\int\left|\widehat{F}(\xi)-F_{k}(\xi)\right| \mathrm{d} \xi<\delta / 2 b$, so that $I_{k} \leq \delta$ for any $k>\bar{k}$.

We finally prove that $\int_{\mathcal{B}} \widehat{F}[Z n(x)] \cdot 1_{\{z>x\}} \mathrm{d} x \in \int_{\mathcal{B}} F[Z n(x)] \cdot 1_{\{z>x\}} \mathrm{d} x$. For any $z \in \mathcal{B}$, we have

$$
\begin{aligned}
\int_{\mathcal{B}} \widehat{F}[Z n(x)] \cdot 1_{\{z>x\}} \mathrm{d} x & =\int \widehat{F}(\xi) \cdot \mathcal{M}_{\{x: x<z, \xi \leq Z n(x)<\xi+\mathrm{d} \xi, Z n(x) \neq 0\}} \mathrm{d} \xi+0 \cdot \mathcal{M}_{\{x: x<z, Z n(x)=0\}} \\
\int_{\mathcal{B}} F[Z n(x)] \cdot 1_{\{z>x\}} \mathrm{d} x & =\int F(\xi) \cdot \mathcal{M}_{\{x: x<z, \xi \leq Z n(x)<\xi+\mathrm{d} \xi, Z n(x) \neq 0\}} \mathrm{d} \xi+\int F(0) \cdot \mathcal{M}_{\{x: x<z,: Z n(x)=0\}} \mathrm{d} \xi
\end{aligned}
$$

where $\mathcal{M}_{\mathcal{E}}$ denotes the measure of a set $\mathcal{E} \subseteq \mathcal{B}$. For any $z \in \mathcal{B}$,

$$
\int_{\mathcal{B}} F[Z n(x)] \cdot 1_{\{z>x\}} \mathrm{d} x=\int_{\mathcal{B}} \widehat{F}[Z n(x)] \cdot 1_{\{z>x\}} \mathrm{d} x+\int F(0) \cdot \mathcal{M}_{\{x: x<z, Z n(x)=0\}} \mathrm{d} \xi
$$

The last term in the RHS is equal to either $\{0\}$ if $\mathcal{M}_{\{x: x<z, Z n(x)=0\}}=0$ or the interval $[-m / s, m]$ where $m=\mathcal{M}_{\{x: x<z, Z n(x)=0\}}$ if $\mathcal{M}_{\{x: x<z, Z n(x)=0\}}>0$. It is then clear that $\int_{\mathcal{B}} F[Z n(x)] \cdot 1_{\{z>x\}} \mathrm{d} x \ni$ $\int_{\mathcal{B}} \widehat{F}[Z n(x)] \cdot 1_{\{z>x\}} \mathrm{d} x$.

Given these lemmas, for any $n \in \mathcal{N}$ and $U \in \mathbb{R}$, we get

$$
\begin{aligned}
\lim _{k \rightarrow \infty} \lim _{l \rightarrow \infty} O_{k l} n(z) & =\lim _{k \rightarrow \infty} \lim _{l \rightarrow \infty} \int_{\mathcal{B}} F_{k}\left[U-\Lambda_{0}-\Lambda_{l} n(x)\right] \cdot 1_{\{z>x\}} \mathrm{d} x \\
& =\lim _{k \rightarrow \infty} \int_{\mathcal{B}} F_{k}\left[U-\Lambda_{0}-\left(\lim _{l \rightarrow \infty} \Lambda_{l} n\right)(x)\right] \cdot 1_{\{z>x\}} \mathrm{d} x \\
& =\lim _{k \rightarrow \infty} \int_{\mathcal{B}} F_{k}\left[U-\Lambda_{0}-\Lambda n(x)\right] \cdot 1_{\{z>x\}} \mathrm{d} x \\
& =\int_{\mathcal{B}} \widehat{F}\left[U-\Lambda_{0}-\Lambda n(x)\right] \cdot 1_{\{z>x\}} \mathrm{d} x
\end{aligned}
$$


where the second equality stems from the continuity of $F_{k}$, the third one from Lemma 13, and

the last one from Lemma 14. Also, from Lemma 14, we have $\int_{\mathcal{B}} \widehat{F}\left[U-\Lambda_{0}-\Lambda n(x)\right] \cdot 1_{\{z>x\}} \mathrm{d} x \in$ $\int_{\mathcal{B}} F\left[U-\Lambda_{0}-\Lambda n(x)\right] \cdot 1_{\{z>x\}} \mathrm{d} x$. So, $\lim _{k \rightarrow \infty} \lim _{l \rightarrow \infty} O_{k l} n(z) \in O n(z)$. Applying this to the fixed point solution $n_{\infty}^{*}=\lim _{k \rightarrow \infty} \lim _{l \rightarrow \infty} n_{k l}^{*}$ and $U_{\infty}^{*}=\lim _{k \rightarrow \infty} \lim _{l \rightarrow \infty} U_{k l}^{*} n_{k l}^{*}$, we get $n_{\infty}^{*}=\lim _{k \rightarrow \infty} \lim _{l \rightarrow \infty} O_{k l} n_{\infty}^{*} \in$ $O n_{\infty}^{*}$. Hence, $n_{\infty}^{*}$ is a fixed point of $O$, which proves Proposition 2.

\section{Appendix C: Monocentric city}

The proof makes uses of the following lemma.

Lemma 15 (i) $S_{M}$ is convex in $[0, \bar{b})$ then concave in $\left[\bar{b}, b_{1}\right)$ for some $\bar{b} \in\left[0, b_{1}\right)$ with $S_{M}^{\prime}(0)=0$. (ii) $S_{\mathcal{Y}}$ is convex in $\left(b_{1}, b\right]$. (iii) $S_{M} \in C^{1}$ in the interval of $[0, b]$ with $S_{M}^{\prime}\left(b_{1}\right)<0$ and $S_{M}^{\prime \prime}\left(b_{1}-0\right)<0<$ $S_{M}^{\prime \prime}\left(b_{1}+0\right)$.

Proof. (i) The access measure $T_{M}$ given by (18) can be written as

$$
T_{M}^{1-\sigma}(z)=\frac{2}{(\sigma-1) \tau s}\left\{1-e^{(1-\sigma) \tau b_{1}} \cosh [(\sigma-1) \tau z]\right\}
$$

Because $-\cosh (z)$ is strictly concave and decreasing for $z \geq 0$, so is $T_{M}^{1-\sigma}$. As $\left(\ln T_{M}^{1-\sigma}\right)^{\prime \prime}=$ $\left[\left(T_{M}^{1-\sigma}\right)^{\prime \prime} T_{M}^{1-\sigma}-\left(T_{M}^{1-\sigma}\right)^{\prime 2}\right] / T_{M}^{2(1-\sigma)}<0, \ln T_{M}^{1-\sigma}$ is strictly concave and decreasing for $z \geq 0$, and so is $-\ln T_{M}(z)$. Differentiating $G_{M}$ as given by (19), we successively get

$$
\begin{aligned}
G_{M}^{\prime}(z) & =\frac{1}{\sigma s}\left[-\sigma \tau \int_{-b_{1}}^{z} \frac{e^{-\sigma \tau(z-y)}}{T_{M}^{1-\sigma}(y)} \mathrm{d} y+\sigma \tau \int_{z}^{b_{1}} \frac{e^{-\sigma \tau(y-z)}}{T_{M}^{1-\sigma}(y)} \mathrm{d} y\right] \\
G_{M}^{\prime \prime}(z) & =\frac{\tau}{s}\left[\sigma \tau \int_{-b_{1}}^{z} \frac{e^{-\sigma \tau(z-y)}}{T_{M}^{1-\sigma}(y)} \mathrm{d} y+\sigma \tau \int_{z}^{b_{1}} \frac{e^{-\sigma \tau(y-z)}}{T_{M}^{1-\sigma}(y)} \mathrm{d} y-\frac{2}{T_{M}^{1-\sigma}(z)}\right] \\
& =\frac{\tau}{s}\left[s \tau \sigma^{2} G_{M}(z)-\frac{2}{T_{M}^{1-\sigma}(z)}\right]
\end{aligned}
$$

Given the above expression of $G_{M}^{\prime \prime}$ and the fact $2 / T_{M}^{1-\sigma}$ is strictly increasing for $z \geq 0$, the curve $s \tau \sigma^{2} G_{M}$ is convex (resp. concave) when above (resp. below) the curve $2 / T_{M}^{1-\sigma}$. Two possibilities actually arise: $s \tau \sigma^{2} G_{M}$ is either concave and below the curve $2 / T_{M}^{1-\sigma}$ or there exists some $\bar{b} \in\left(0, b_{1}\right)$ such that $s \tau \sigma^{2} G_{M}$ is convex on $[0, \bar{b}]$ and concave on $\left[\bar{b}, b_{1}\right]$ with $\bar{b}$ necessarily corresponding to the single intersection point of $s \tau \sigma^{2} G_{M}$ with the curve $2 / T_{M}^{1-\sigma}$. See the illustration in Figure A1. It can also be shown that $S_{M}^{\prime}(0)=G_{M}^{\prime}(0)=\left(-\ln T_{M}\right)^{\prime}(0)=0$. 
(ii) We now prove that $S_{M}$ is convex in $\left(b_{1}, b\right]$. For $x \in\left(b_{1}, b\right]$, we have

$$
\begin{aligned}
G_{M}(x) & =\frac{1}{\sigma s} \int_{-b_{1}}^{b_{1}} \frac{e^{-\sigma \tau(x-y)}}{T_{M}^{1-\sigma}(y)} \mathrm{d} y \\
G_{M}^{\prime}(x) & =-\frac{\tau}{s} \int_{-b_{1}}^{b_{1}} \frac{e^{-\sigma \tau(x-y)}}{T_{M}^{1-\sigma}(y)} \mathrm{d} y<0 \\
G_{M}^{\prime \prime}(x) & =\frac{\sigma \tau^{2}}{s} \int_{-b_{1}}^{b_{1}} \frac{e^{-\sigma \tau(x-y)}}{T_{M}^{1-\sigma}(y)} \mathrm{d} y>0
\end{aligned}
$$

and

$$
\ln T_{M}(x)=\tau x+\frac{1}{1-\sigma} \ln \left(\frac{1}{s} \int_{-b_{1}}^{b_{1}} e^{-(1-\sigma) \tau y} \mathrm{~d} y\right)
$$

which is linear in $x$. Hence, $S_{M}$ is convex in $\left(b_{1}, b\right]$.

(iii) We showed that $-\ln T_{M}(z)$ is concave, decreasing and $C^{1}$ on $[0, b]$. For $z \rightarrow b_{1}-0$, we have

$$
\begin{aligned}
& G_{M}^{\prime}\left(b_{1}-0\right)=-\frac{\tau}{s} \int_{-b_{1}}^{b_{1}} \frac{e^{-\sigma \tau\left(b_{1}-y\right)}}{T_{M}^{1-\sigma}(y)} \mathrm{d} y<0 \\
& G_{M}^{\prime \prime}\left(b_{1}-0\right)=\frac{\tau}{s}\left[\sigma \tau \int_{-b_{1}}^{b_{1}} \frac{e^{-\sigma \tau\left(b_{1}-y\right)}}{T_{M}^{1-\sigma}(y)} \mathrm{d} y-\frac{2}{T_{M}^{1-\sigma}\left(b_{1}\right)}\right]<0
\end{aligned}
$$

as $G_{M}$ is concave in $z=b_{1}$ by part (i). For $z \rightarrow b_{1}+0$, we have

$$
\begin{aligned}
& G_{M}^{\prime}\left(b_{1}+0\right)=-\frac{\tau}{s} \int_{-b_{1}}^{b_{1}} \frac{e^{-\sigma \tau\left(b_{1}-y\right)}}{T_{M}^{1-\sigma}(y)} \mathrm{d} y<0 \\
& G_{M}^{\prime \prime}\left(b_{1}+0\right)=\frac{\sigma \tau^{2}}{s} \int_{-b_{1}}^{b_{1}} \frac{e^{-\sigma \tau\left(b_{1}-y\right)}}{T_{M}^{1-\sigma}(y)} \mathrm{d} y>0
\end{aligned}
$$

by using expression (61). Therefore, $S_{M}^{\prime}\left(b_{1}-0\right)=S_{M}^{\prime}\left(b_{1}+0\right)$ but $S_{M}^{\prime \prime}\left(b_{1}-0\right)<0<S_{M}^{\prime \prime}\left(b_{1}+0\right)$. So, $S_{M}$ is $C^{1}$ on $[0, b]$. It also appears that $S_{M}^{\prime}\left(b_{1}\right)<0$.

\section{INSERT FIGURE A1 HERE}

Figure A1: Concavity and convexity of $G_{M}$.

Note: The figure represents $s \tau \sigma^{2} G_{M}$ and $2 / T_{M}$ with plain and dashed curves respectively.

Parameters: left panel: $\sigma=3, \tau=1, s=1$; right panel $\sigma=6, \tau=2, s=1$.

We now show that the equilibrium conditions $\Psi_{b}(y) \geq \Psi_{r}(y)$ for all $y \in\left[0, b_{1}\right]$ and $\Psi_{r}(x) \geq \Psi_{b}(x)$ for all $x \in\left[b_{1}, b\right]$ can be reduced to $\Psi_{b}(0) \geq \Psi_{r}(0)$ and $\Psi_{b}(b) \leq \Psi_{r}(b)=0$ respectively.

Proof. In the interval of $[0, b]$, the bid rents of residents and firms are given by

$$
\begin{aligned}
& \Psi_{r}(y)=\Psi_{r}(0)-t y \\
& \Psi_{b}(y)=\frac{1}{s}\left[(\alpha+\beta) S_{M}(y)+t y+\left(\alpha \ln \frac{\alpha \rho}{\gamma \mathrm{e}}+\beta \ln \frac{\beta \rho}{\gamma \mathrm{e}}-U_{0}+\bar{c}_{0}\right)\right]
\end{aligned}
$$


Given Lemma $15(\mathrm{i}), \Psi_{b}$ is convex in $[0, \bar{b})$ then concave in $\left[\bar{b}, b_{1}\right)$ for some $\bar{b} \in\left[0, b_{1}\right)$ with $\Psi_{b}^{\prime}(0)=t$. Therefore $\Psi_{b}(y)-\Psi_{r}(y)$ is quasiconcave on $\left[0, b_{1}\right]$. As $\Psi_{b}\left(b_{1}\right)=\Psi_{r}\left(b_{1}\right)$, the equilibrium condition $\Psi_{b}(y) \geq \Psi_{r}(y)$ for all $y \in\left[0, b_{1}\right]$ reduces to $\Psi_{b}(0) \geq \Psi_{r}(0)$. According to Lemma $15(\mathrm{ii}), \Psi_{b}(z)$ is convex for $z \in\left[b_{1}, b\right]$. As $\Psi_{r}(z)$ is linear and $\Psi_{b}\left(b_{1}\right)=\Psi_{r}\left(b_{1}\right)$, the equilibrium condition $\Psi_{b}(x) \leq \Psi_{r}(x)$ for all $x \in\left[b_{1}, b\right]$ reduces to $\Psi_{b}(b) \leq \Psi_{r}(b)=0$.

\section{Appendix D: Integrated city}

We show that $\left|S_{I}^{\prime}(b)\right| \geq\left|S_{I}^{\prime}(z)\right|, \forall z \in[0, b]$.

Proof. Given the similarity of expression of $T_{I}(25)$ with that corresponding to the monocentric case (18), the argument used in Appendix A showing that $\ln T_{M}^{1-\sigma}$ is strictly concave and decreasing in $z$ in Appendix A, can be applied here. Thus, $-\ln \left(T_{I}\right)$ is strictly concave and decreasing for $y \geq 0$.

By differentiating $G_{I}(z)$ as given by expression (26), we get

$$
\begin{aligned}
G_{I}^{\prime}(z) & =\frac{1}{\sigma(1+s)}\left[-\sigma \tau \int_{-b}^{z} \frac{e^{-\sigma \tau(z-y)}}{T_{I}^{1-\sigma}(y)} \mathrm{d} y+\sigma \tau \int_{z}^{b} \frac{e^{-\sigma \tau(y-z)}}{T_{I}^{1-\sigma}(y)} \mathrm{d} y\right] \\
& =\frac{\tau}{1+s}\left[\int_{-b}^{z} \frac{e^{-\sigma \tau(z-y)}}{T_{I}^{1-\sigma}(y)} \mathrm{d} y+\int_{z}^{b} \frac{e^{-\sigma \tau(y-z)}}{T_{I}^{1-\sigma}(y)} \mathrm{d} y-2 \int_{-b}^{z} \frac{e^{-\sigma \tau(z-y)}}{T_{I}^{1-\sigma}(y)} \mathrm{d} y\right] \\
& =\frac{\tau}{1+s}\left[\sigma(1+s) G_{I}(z)-2 \int_{-b}^{z} \frac{e^{-\sigma \tau(z-y)}}{T_{I}^{1-\sigma}(y)} \mathrm{d} y\right] \\
G_{I}^{\prime \prime}(z) & =\frac{\tau}{1+s}\left[(1+s) \tau \sigma^{2} G_{I}(z)-\frac{2}{T_{I}^{1-\sigma}(z)}\right]
\end{aligned}
$$

It suffices to show that $G_{I}^{\prime}(b)+G_{I}^{\prime}(z) \leq 0$ in order to have

$$
\left|G_{I}^{\prime}(b)\right| \geq\left|G_{I}^{\prime}(z)\right|, \quad \forall z \in[0, b]
$$


as $G_{I}^{\prime}(b)<0$. To show that $G_{I}^{\prime}(b)+G_{I}^{\prime}(z) \leq 0$, we develop $G_{I}^{\prime}(b)+G_{I}^{\prime}(z)$ into

$$
\begin{aligned}
& \frac{\tau}{1+s}\left[\sigma(1+s)\left[G_{I}(b)+G_{I}(z)\right]-2 \int_{-b}^{b} \frac{e^{-\sigma \tau(b-y)}}{T_{I}^{1-\sigma}(y)} \mathrm{d} y-2 \int_{-b}^{z} \frac{e^{-\sigma \tau(z-y)}}{T_{I}^{1-\sigma}(y)} \mathrm{d} y\right] \\
& =\frac{\tau}{1+s}\left[\int_{-b}^{b} \frac{e^{-\sigma \tau(b-y)}}{T_{I}^{1-\sigma}(y)} \mathrm{d} y+\int_{-b}^{z} \frac{e^{-\sigma \tau(z-y)}}{T_{I}^{1-\sigma}(y)} \mathrm{d} y+\int_{z}^{b} \frac{e^{-\sigma \tau(y-z)}}{T_{I}^{1-\sigma}(y)} \mathrm{d} y-2 \int_{-b}^{b} \frac{e^{-\sigma \tau(b-y)}}{T_{I}^{1-\sigma}(y)} \mathrm{d} y-2 \int_{-b}^{z} \frac{e^{-\sigma \tau(z-y)}}{T_{I}^{1-\sigma}(y)} \mathrm{d} y\right] \\
& =\frac{\tau}{1+s}\left[\int_{z}^{b} \frac{e^{-\sigma \tau(y-z)}}{T_{I}^{1-\sigma}(y)} \mathrm{d} y-\int_{-b}^{b} \frac{e^{-\sigma \tau(b-y)}}{T_{I}^{1-\sigma}(y)} \mathrm{d} y-\int_{-b}^{z} \frac{e^{-\sigma \tau(z-y)}}{T_{I}^{1-\sigma}(y)} \mathrm{d} y\right] \\
& =\frac{\tau}{1+s}\left[\int_{z}^{b} \frac{e^{-\sigma \tau(y-z)}}{T_{I}^{1-\sigma}(y)} \mathrm{d} y-\int_{-b}^{z} \frac{e^{-\sigma \tau(b-y)}}{T_{I}^{1-\sigma}(y)} \mathrm{d} y-\int_{z}^{b} \frac{e^{-\sigma \tau(b-y)}}{T_{I}^{1-\sigma}(y)} \mathrm{d} y-\int_{-b}^{z} \frac{e^{-\sigma \tau(z-y)}}{T_{I}^{1-\sigma}(y)} \mathrm{d} y\right] \\
& =\frac{\tau}{1+s}\left[\int_{z}^{b} \frac{e^{-\sigma \tau(y-z)}-e^{-\sigma \tau(b-y)}}{T_{I}^{1-\sigma}(y)} \mathrm{d} y-\int_{-b}^{z} \frac{e^{-\sigma \tau(b-y)}}{T_{I}^{1-\sigma}(y)} \mathrm{d} y-\int_{-b}^{z} \frac{e^{-\sigma \tau(z-y)}}{T_{I}^{1-\sigma}(y)} \mathrm{d} y\right]
\end{aligned}
$$

As $T_{I}^{1-\sigma}$ is decreasing for $z \geq 0$, we have $\int_{z}^{b}\left(e^{-\sigma \tau(y-z)}-e^{-\sigma \tau(b-y)}\right) / T_{I}^{1-\sigma}(y) \mathrm{d} y \leq 0, \forall z \geq 0$, because the increasing function $e^{-\sigma \tau(y-z)}$ is the reflected image of $e^{-\sigma \tau(b-y)}$ over the line $y=(z+b) / 2$. This proves $G_{I}^{\prime}(b)+G_{I}^{\prime}(z) \leq 0$. Finally, using the fact that $-\ln T_{I}$ is strictly concave and decreasing and relation (62) leads to

$$
\left|S_{I}^{\prime}(b)\right| \geq\left|S_{I}^{\prime}(z)\right|, \forall z \in[0, b]
$$

Also, note that $S_{I}^{\prime}(b)<0$ as both $G_{I}^{\prime}(b)$ and $-\left(\ln T_{I}\right)^{\prime}(b)$ are negative. Therefore, $S_{I}(z)$ is concave and decreasing in the neighborhood of $z=b$.

We next prove that $t_{I}$ is an increasing function of $\tau$.

Proof. We need to show that $S_{I}^{\prime}(b)$ is a decreasing function of $\tau$. First, it can be shown that $d\left[-(\ln T)^{\prime}(b)\right] / d \tau=-1$. Second, by using expression $(26)$ we have $G_{I}^{\prime}(b)=-\tau /(1+s) \int_{-b}^{b} e^{-\sigma \tau(b-z)} / T_{I}^{1-\sigma}(z) \mathrm{d} z$ and

$$
\frac{d}{d \tau} G_{I}^{\prime}(b)=-\frac{1}{1+s} \int_{-b}^{b} \frac{e^{-\sigma \tau(b-z)}}{T_{I}^{1-\sigma}(z)} \mathrm{d} z+\frac{\sigma \tau}{1+s} \int_{-b}^{b} \frac{(b-z) e^{-\sigma \tau(b-z)}}{T_{I}^{1-\sigma}(z)} \mathrm{d} z-\frac{\tau}{1+s} \int_{-b}^{b} e^{-\sigma \tau(b-z)} \frac{d}{d \tau} T_{I}^{\sigma-1}(z) \mathrm{d} z
$$


As $\mathrm{d}\left[T_{I}^{1-\sigma}(z)\right] / \mathrm{d} \tau<0$, it suffices to show that the sum of the first two terms is negative. We have

$$
\begin{aligned}
& -\frac{1}{1+s} \int_{-b}^{b} \frac{e^{-\sigma \tau(b-z)}}{T_{I}^{1-\sigma}(z)} \mathrm{d} z+\frac{\sigma \tau}{1+s} \int_{-b}^{b} \frac{(b-z) e^{-\sigma \tau(b-z)}}{T_{I}^{1-\sigma}(z)} \mathrm{d} z \\
& =\frac{1}{1+s} \int_{-b}^{b}[(b-z) \sigma \tau-1] e^{-\sigma \tau(b-z)} T_{I}^{\sigma-1}(z) \mathrm{d} z \\
& =\frac{1}{(1+s) \sigma \tau} \int_{0}^{2 \sigma \tau b}(r-1) e^{-r} T_{I}^{\sigma-1}[b-r /(\sigma \tau)] \mathrm{d} r \\
& =\frac{1}{(1+s) \sigma \tau}\left(\int_{0}^{1}+\int_{1}^{2 \sigma \tau b}\right)(r-1) e^{-r} T_{I}^{\sigma-1}[b-r /(\sigma \tau)] \mathrm{d} r \\
& \leq \frac{T_{I}^{\sigma-1}[b-1 /(\sigma \tau)]}{(1+s) \sigma \tau}\left(\int_{0}^{1}+\int_{1}^{2 \sigma \tau b}\right)(r-1) e^{-r} \mathrm{~d} r<0
\end{aligned}
$$

as $T_{I}^{\sigma-1}[b-r /(\sigma \tau)]$ a decreasing function of $r$ and $\int_{0}^{R}(r-1) e^{-r} \mathrm{~d} r<0$.

\section{Appendix E: Cut-offs $t_{M}$ and $t_{I}$}

We prove that $\lim _{s \rightarrow 0} t_{M}<\lim _{s \rightarrow 0} t_{I}$. As $s \longrightarrow 0, t_{M}=\bar{t}_{M}=(\alpha+\beta) \tau$. We need to check that

$$
\left(\ln T_{I}\right)^{\prime}(b)-G_{I}^{\prime}(b)>\tau
$$

when $s \rightarrow 0$. Given the expressions (25), (26) of $T_{I}^{1-\sigma}$ and $G_{I}^{\prime}$, we have

$$
\begin{aligned}
\left(\ln T_{I}\right)^{\prime}(b) & =\left.\frac{1}{1-\sigma} \frac{d}{d z} \ln \left[1-e^{(1-\sigma) \tau b} \cosh [(\sigma-1) \tau z]\right]\right|_{z=b} \\
& =\left.\tau \frac{e^{(1-\sigma) \tau b} \sinh [(\sigma-1) \tau z]}{1-e^{(1-\sigma) \tau b} \cosh [(\sigma-1) \tau z]}\right|_{z=b} \\
& =\tau
\end{aligned}
$$

and

$$
G_{I}^{\prime}(b)=-\frac{(\sigma-1) \tau^{2}}{2} \int_{-b}^{b} \frac{e^{-\sigma \tau(b-y)}}{1-e^{(1-\sigma) \tau b} \cosh [(\sigma-1) \tau y]} \mathrm{d} y
$$

where $G_{I}^{\prime}(b)$ is negative because the denominator of this integrand is positive as

$$
\begin{aligned}
1-e^{(1-\sigma) \tau b} \cosh [(\sigma-1) \tau z] & \geq 1-e^{(1-\sigma) \tau b} \frac{e^{(\sigma-1) \tau b}+e^{-(\sigma-1) \tau b}}{2} \\
& =1-\frac{1+e^{-2(\sigma-1) \tau b}}{2} \\
& =\frac{1-e^{-2(\sigma-1) \tau b}}{2} \\
& >0
\end{aligned}
$$

Hence, we get $\left(\ln T_{I}\right)^{\prime}(b)-G_{I}^{\prime}(b)>\tau$. 


\section{Appendix F: Proof of Corollary 2}

We show that the partially integrated city is a spatial equilibrium for $t=t_{I}-\delta$ and small enough $\delta>0$. By continuity, $S_{P}(z)$ tends to $S_{I}(z)$ for any $z$ when $b_{1}$ tends to $b$. This is also true for $S_{P}^{\prime}(z)$ and $S_{P}^{\prime \prime}(z)$. It is shown in Appendix $\mathrm{D}$ that $S_{I}(z)$ is decreasing and concave in the neighborhood of $z=b$ and $\left|S_{I}^{\prime}(b)\right|>\left|S_{I}^{\prime}(z)\right|, z \in[0, b]$. Hence, we have $S_{P}^{\prime}(z)<0$ and $S_{P}^{\prime \prime}(z)<0$ for $z$ close enough to $b$ while $\left|S_{P}^{\prime}(b)\right|>\left|S_{P}^{\prime}(z)\right|, \forall z \in[0, b]$. By continuity, $\left|S_{P}^{\prime}\left(b_{1}\right)\right|>\left|S_{P}^{\prime}(z)\right|, \forall z \in\left[0, b_{1}\right]$ when $b_{1}$ tends to $b$. In this case, $S_{P}(z)$ is decreasing and concave in $z$ within the intervals $\left[b_{1}, b\right]$ for small $\delta>0$. So, by concavity of $S_{P}(z)$, we compute

$$
\begin{aligned}
\bar{t}_{P} & \equiv \min _{y \in\left[b_{1}, b_{2}\right]} \frac{\alpha+\beta}{1+s} \frac{S_{P}(y)-S_{P}\left(b_{2}\right)}{b_{2}-y}=\frac{\alpha+\beta}{1+s} \frac{S_{P}\left(b_{1}\right)-S_{P}\left(b_{2}\right)}{b_{2}-b_{1}} \\
\widetilde{t}_{P} & \equiv \frac{\alpha+\beta}{1+s} \frac{S_{P}\left(b_{2}\right)-S_{P}(b)}{b-b_{2}} \\
\widehat{t}_{P} & \equiv \frac{\alpha+\beta}{1+s} \max _{z \in\left[0, b_{1}\right)}\left|S_{P}^{\prime}(z)\right|=\frac{\alpha+\beta}{1+s}\left|S_{P}^{\prime}\left(b_{1}\right)\right|
\end{aligned}
$$

while

$$
t=\frac{\alpha+\beta}{1+s} \frac{S_{P}\left(b_{1}\right)-S_{P}\left(b_{2}\right)}{b_{2}-b_{1}}
$$

We now check that $\widehat{t}_{P} \leq t \leq \min \left(\bar{t}_{P}, \widetilde{t}_{P}\right)$. Indeed, $t=\bar{t}_{P}$ and $\widehat{t}_{P} \leq t \leq \widetilde{t}_{P}$ by concavity of $S_{P}(z)$.

\section{References}

[1] Alonso, W. (1964) Location and Land Use, Harvard University Press, Cambridge, MA.

[2] Berliant, M., S.-K. Peng, and P. Wang (2002) Production externalities and urban configuration, Journal of Economic Theory 104, 275-303.

[3] Carlier, G. and I. Ekeland (2007) Equilibrium structure of a bidimensional asymmetric city, Nonlinear Analysis: Real World Applications 8, 725-748.

[4] Fujita, M. and H. Ogawa (1982) Multiple equilibria and structural transition of non-monocentric urban configurations, Regional Science and Urban Economics 12, 161-196.

[5] Lucas, R. and E. Rossi-Hansberg (2002) On the internal structure of cities, Econometrica 70 , 1445-1476. 
[6] Fujita, M., P. Krugman, and T. Mori (1999). On the evolution of hierarchical urban systems, European Economic Review, 43, 209-251.

[7] Ogawa, H. and M. Fujita (1980) Equilibrium land use patterns in a nonmonocentric city, Journal of Regional Science 20, 455-475.

[8] Pflüger, M.P. (2004) A simple, analytically solvable Chamberlinian agglomeration model, Regional Science and Urban Economics 34, 565-573. 


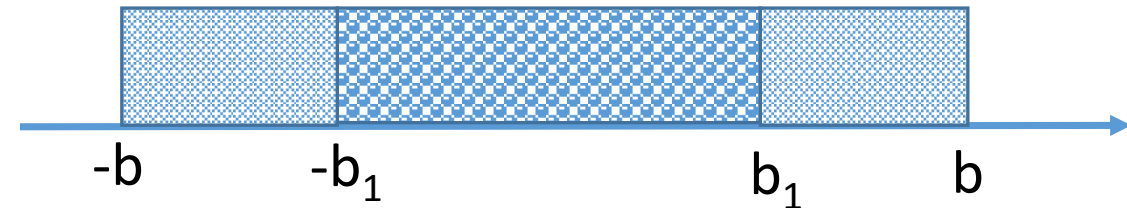

(a) Monocentric city

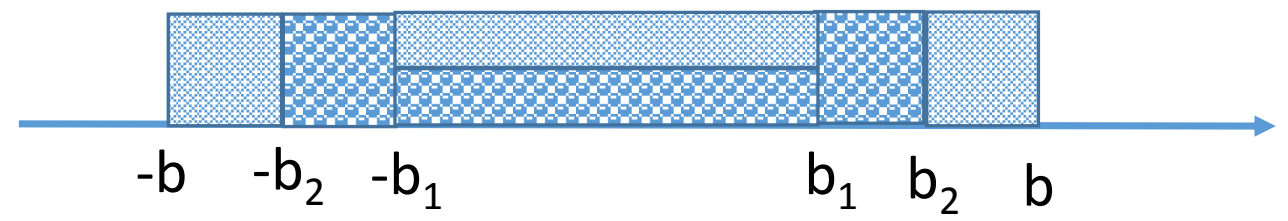

(c) Partially integrated city

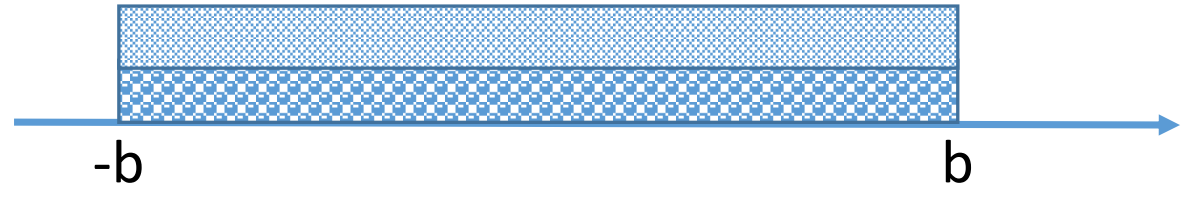

(b) Integrated city

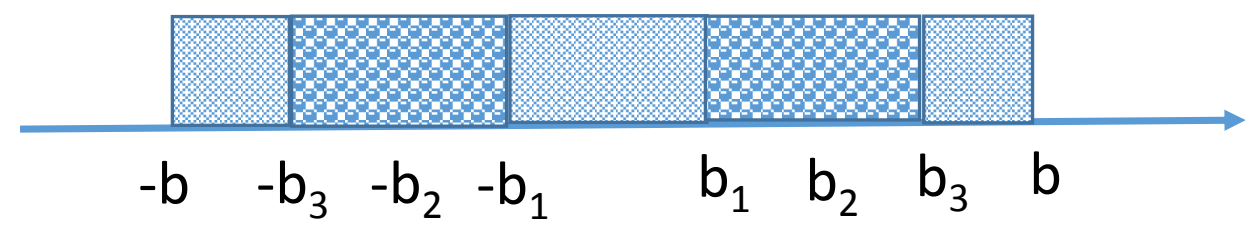

(d) Duocentric city

Figure 1: Spatial urban structures

( 


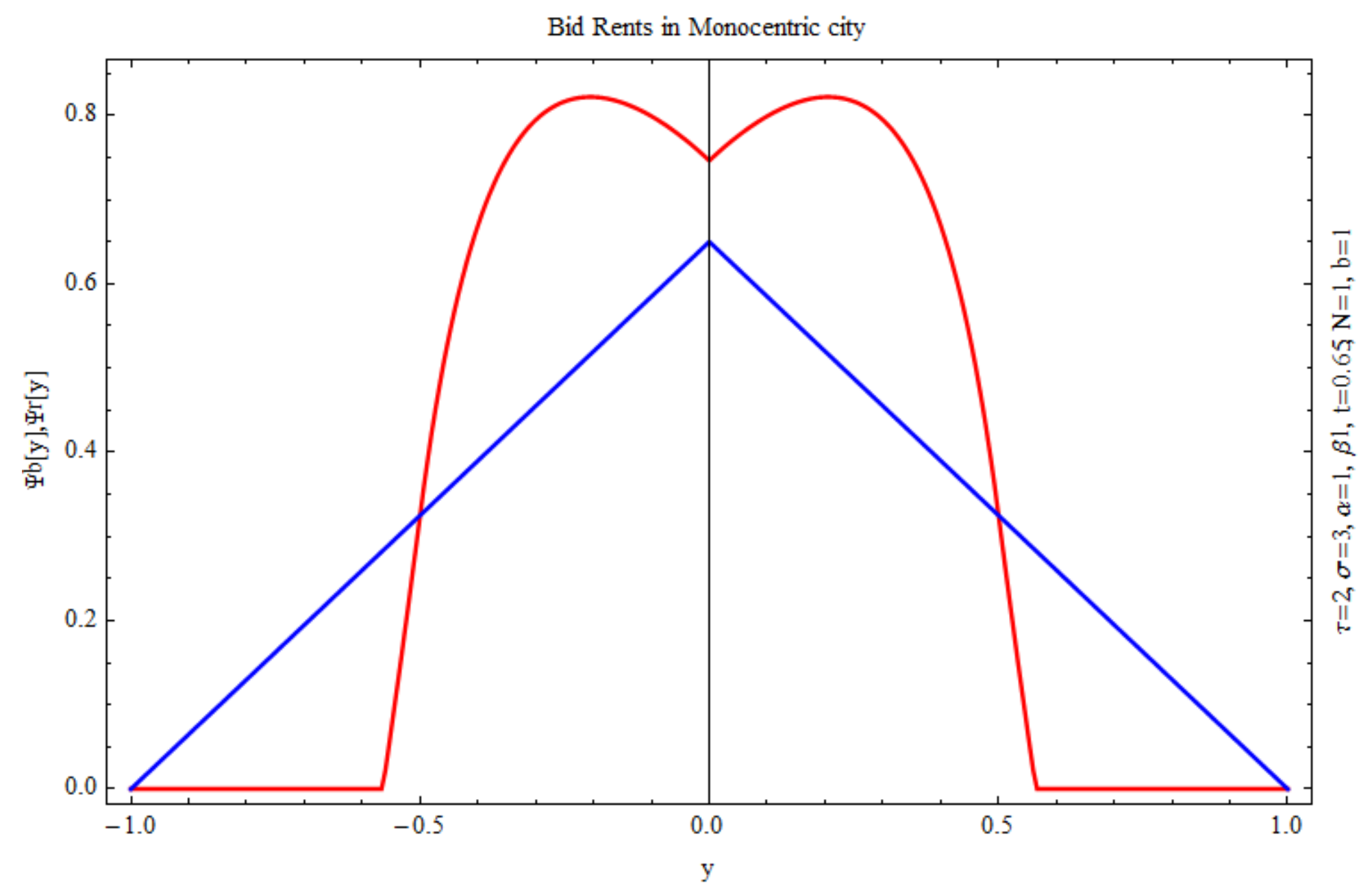

Figure 2: Monocentric city: residential (blue) and business (red) bid rents. 
Bid Rents in Integrated city

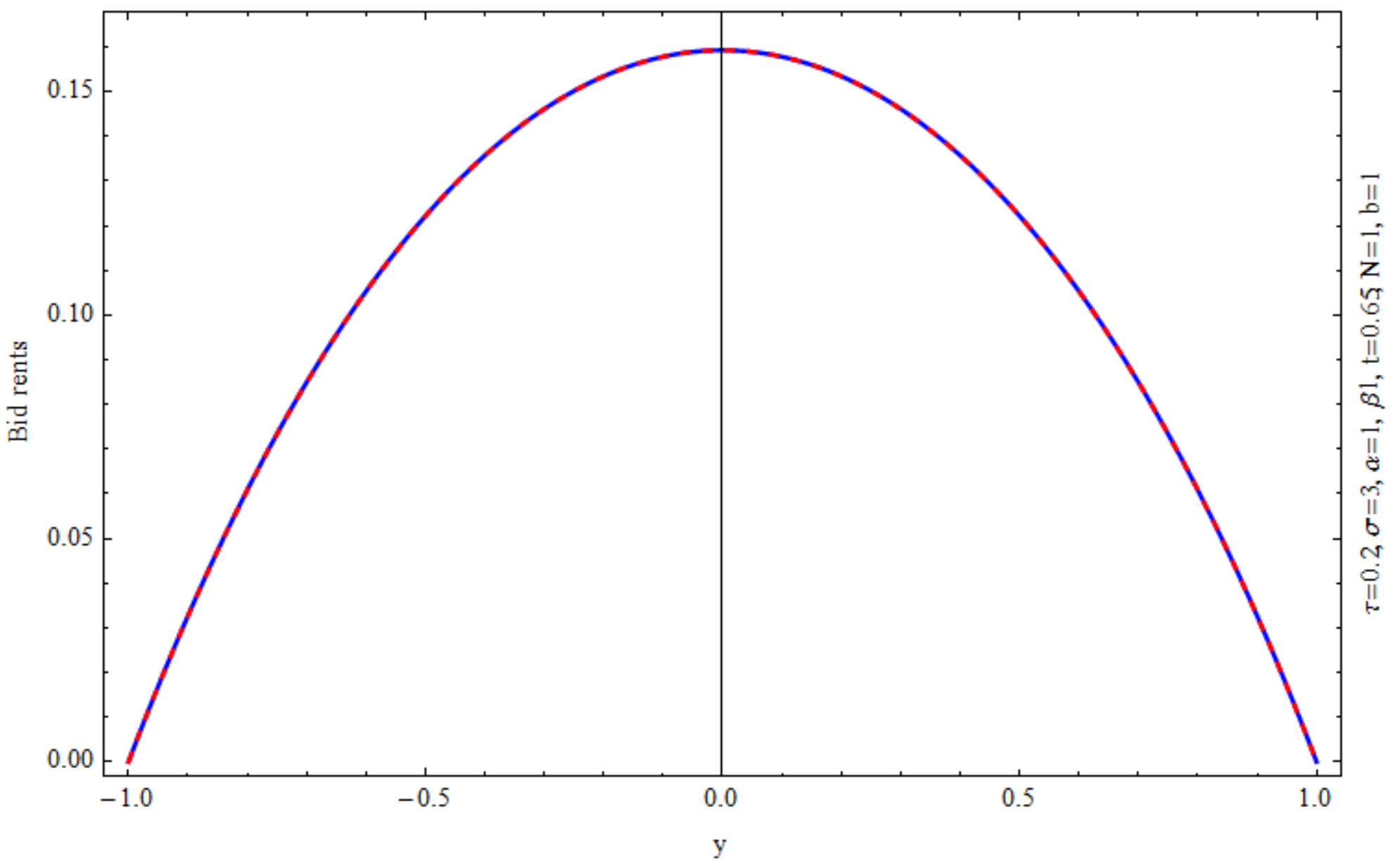

Figure 3: Integrated city: residential (blue) and business (red) bid rents. 


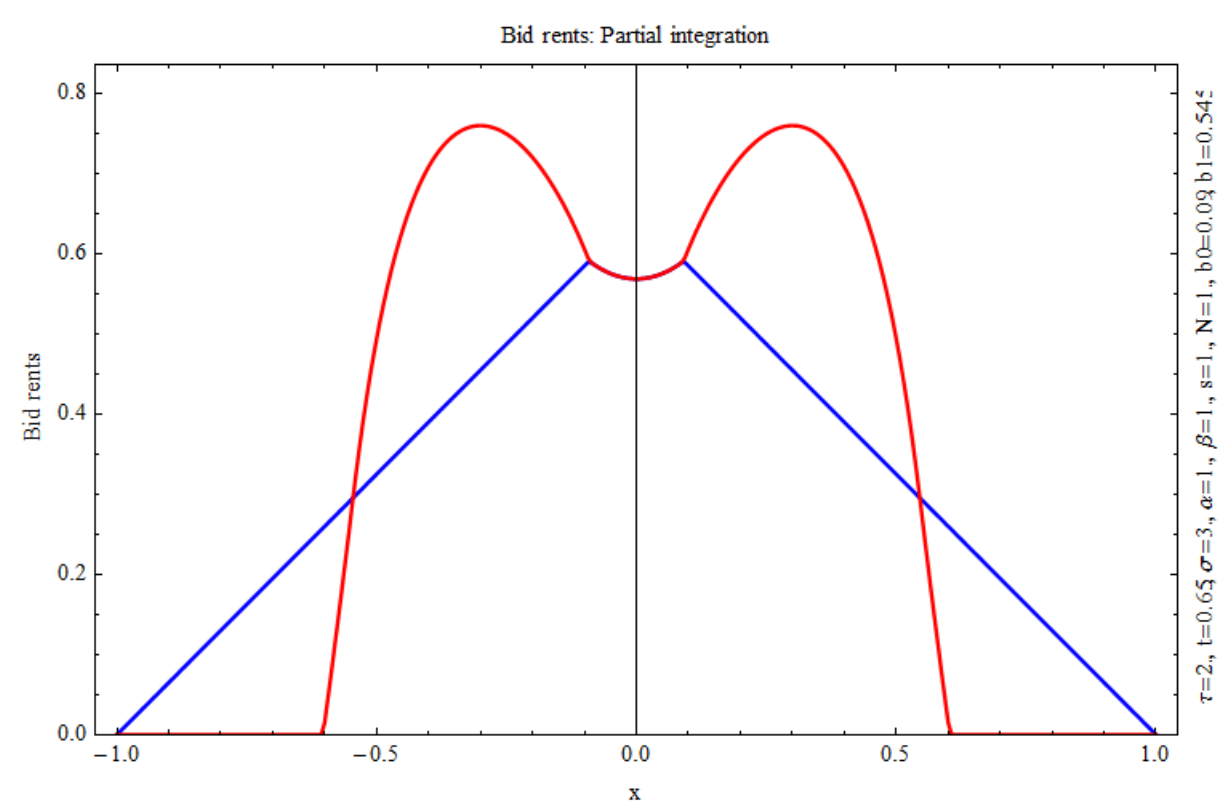

Panel a: balanced use of space, $s=1$

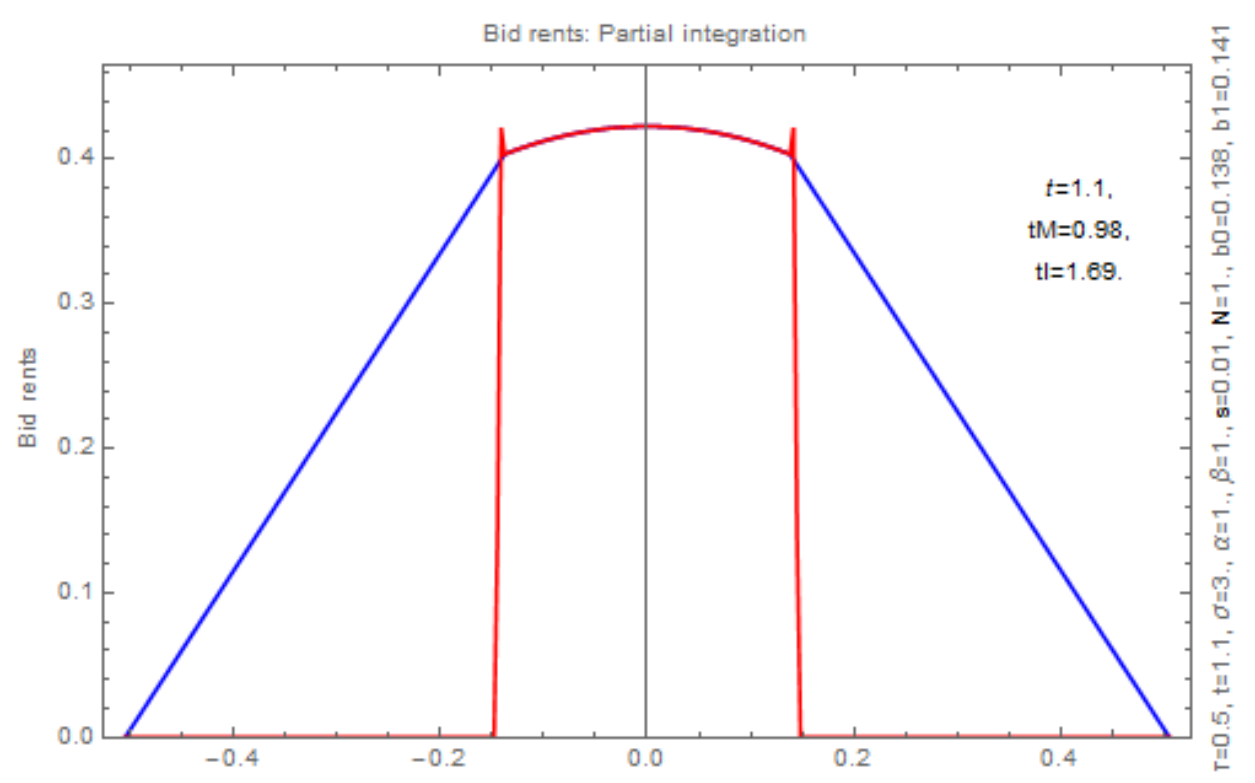

Panel b: unbalanced use of space, $s=0.01$

Figure 4: Partially integrated city: residential (blue) and business (red) bid rents. 


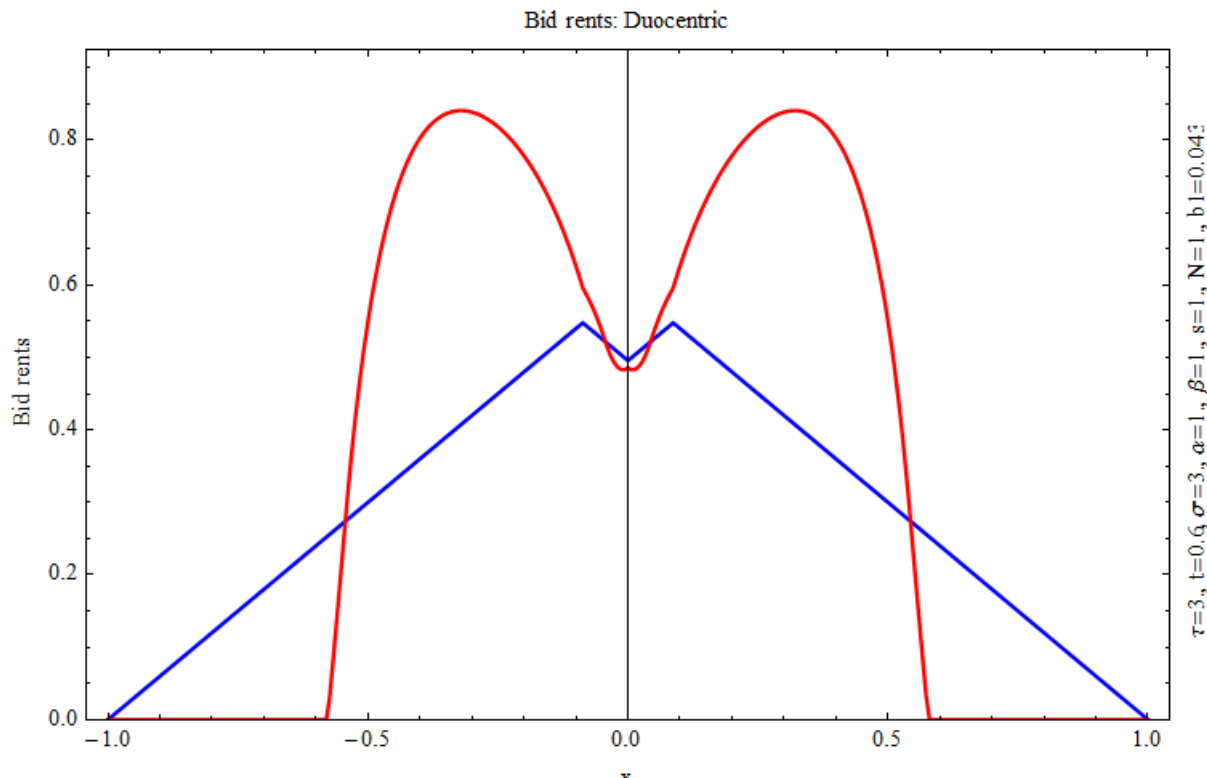

Panel a): Duocentric equilibrium 1

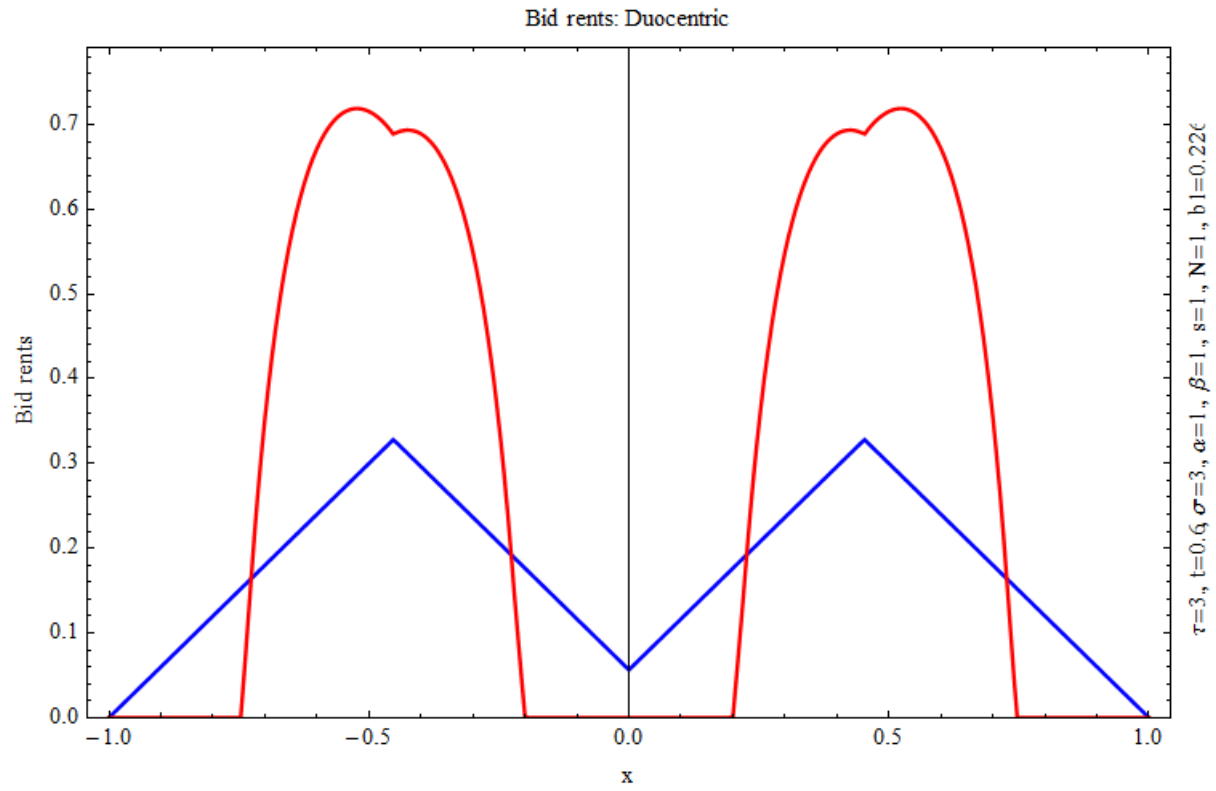

Panel b): Duocentric equilibrium 2

Figure 5: Duocentric city: residential (blue) and business (red) bid rents. 


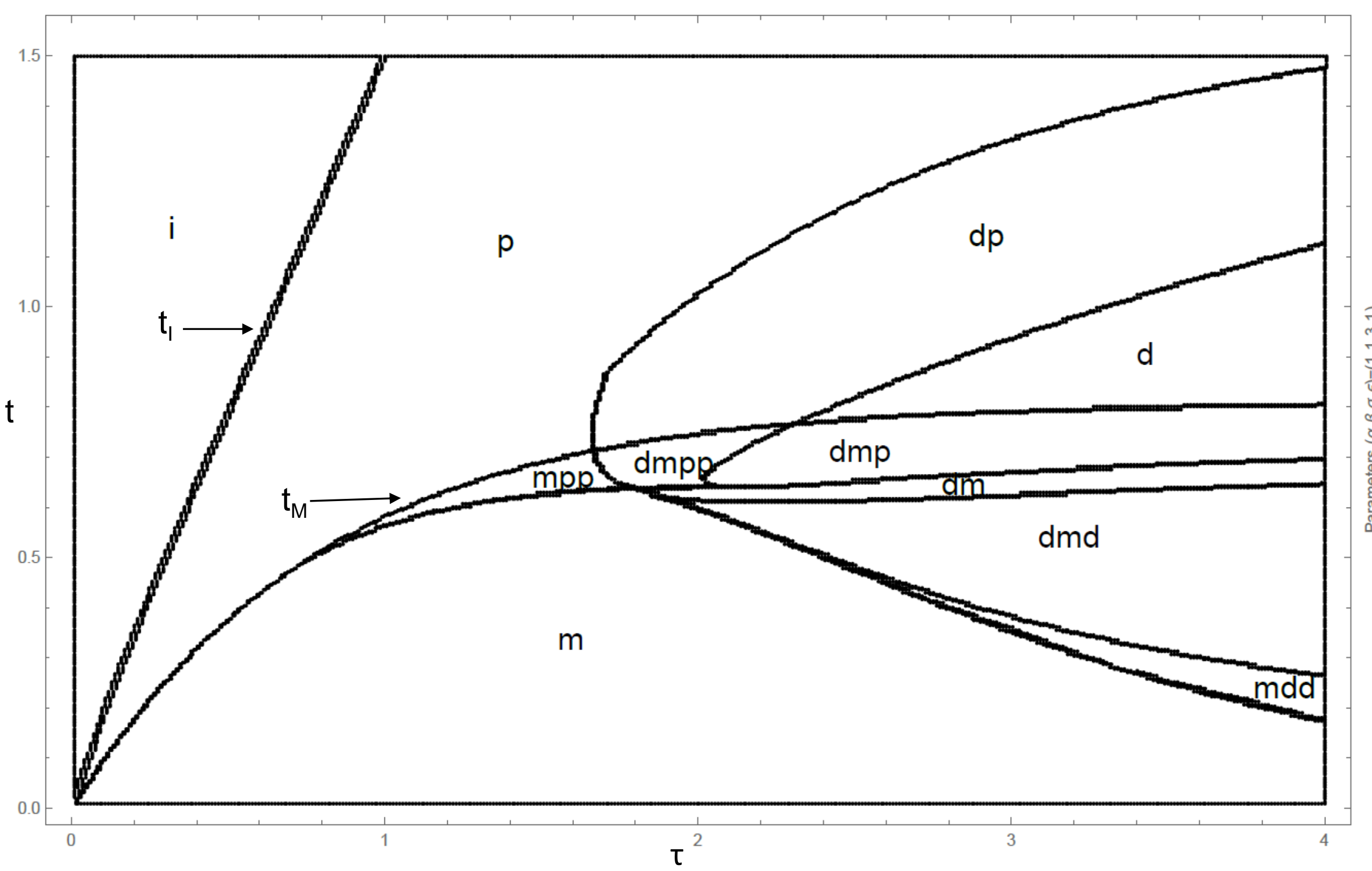

Figure 6: Monocentric $(m)$, partially integrated $(p)$, integrated $(i)$, and duocentric configurations in $(\tau, t)$ plane $(\alpha=\beta=\gamma=s=1$ and $\sigma=3$ ) 

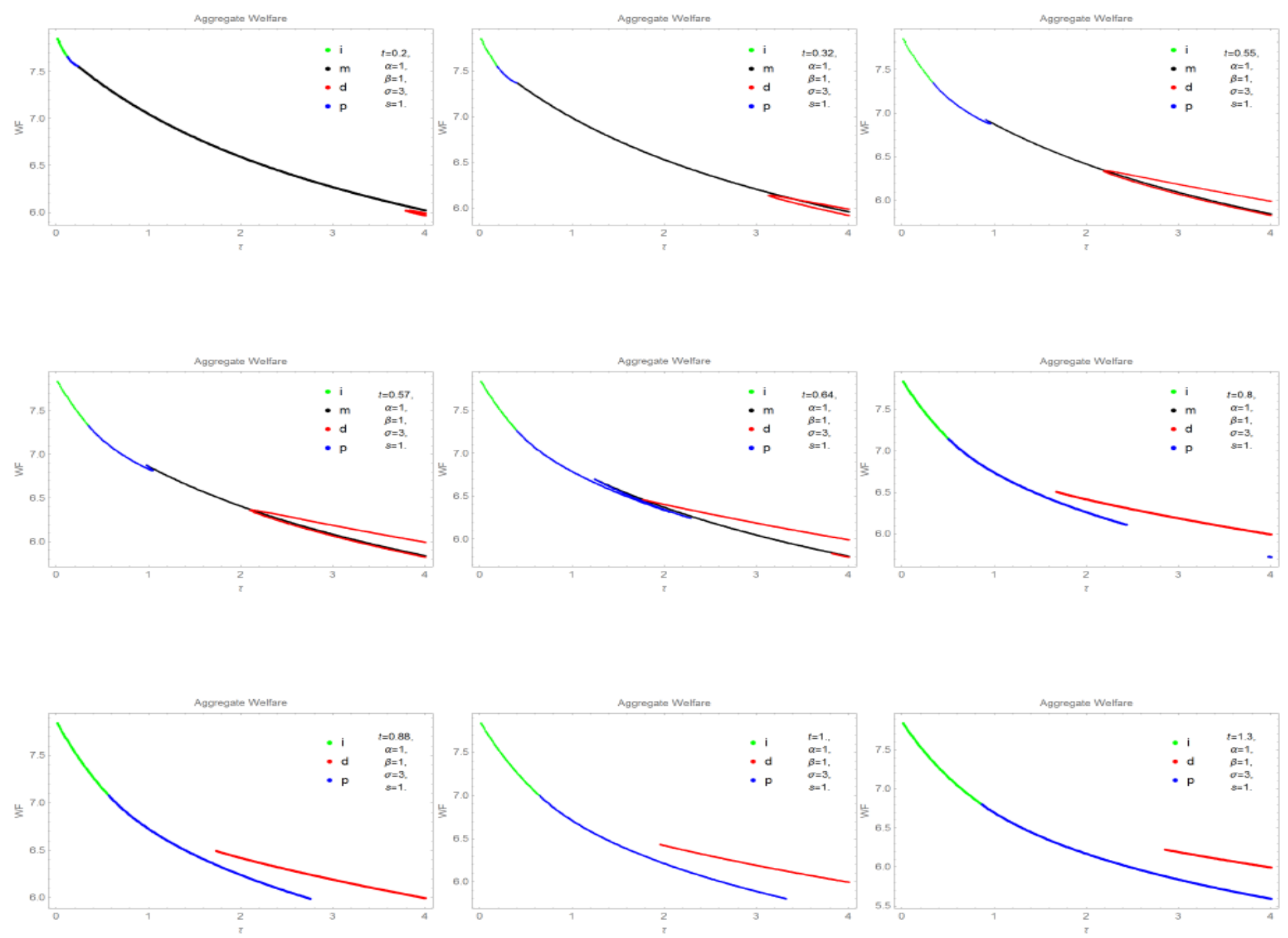

Figure 7: Aggregate welfare of urban configurations in terms of transport cost $\tau$ for various values of commuting cost $t$ 


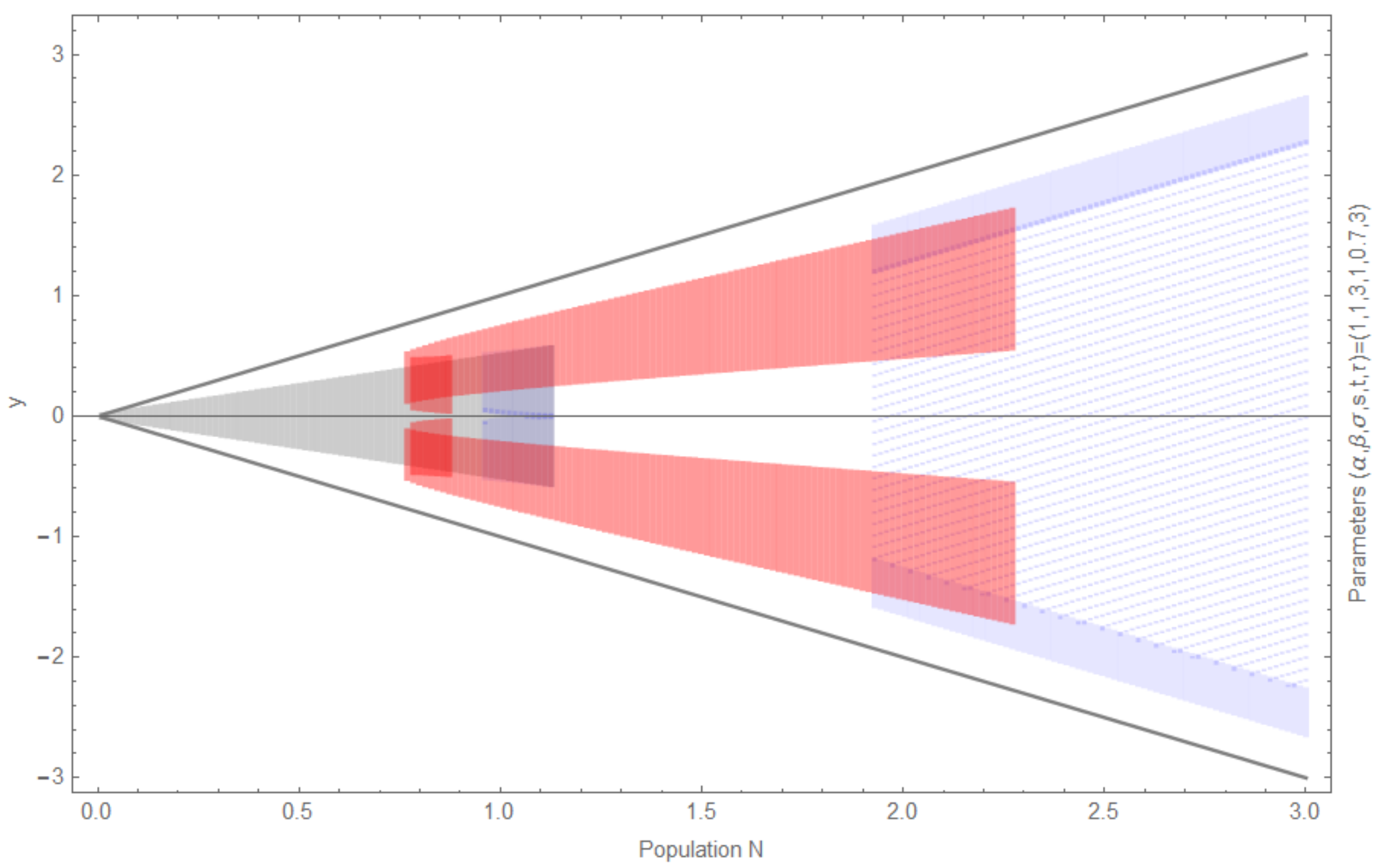

Figure 8: City structure as population size increases

The horizontal axis is population and the vertical axis location. Diagonal straight lines display city borders. Residential districts are represented in white, monocentric business districts in grey, duocentric business districts in red, and the business and the integrated districts of the partially integrated city in plain and dash blue respectively. Overlaps indicate multiple equilibria. 

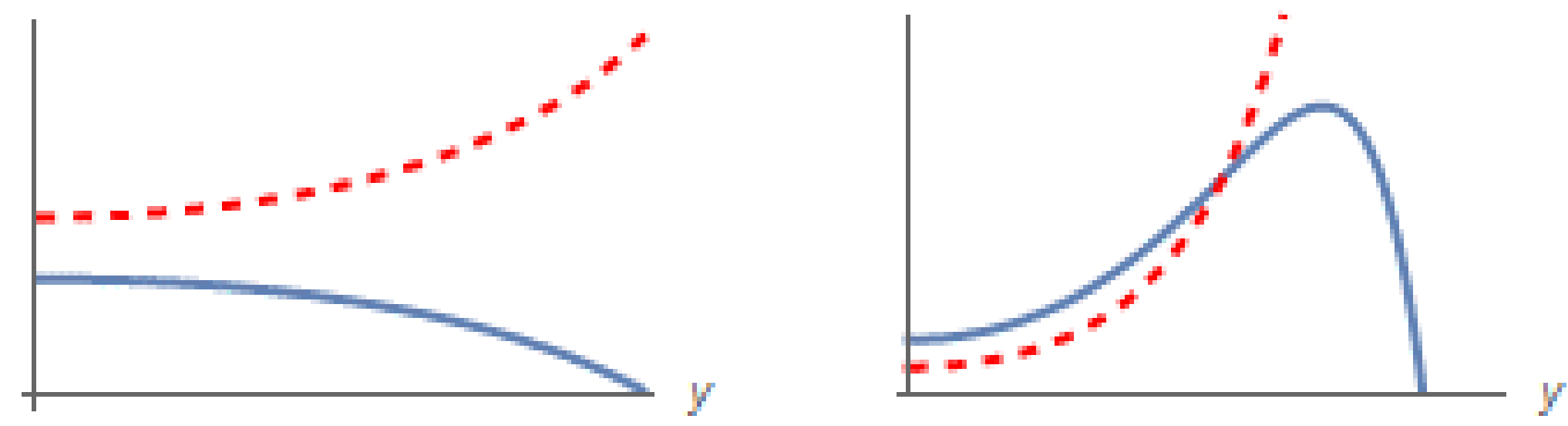

Figure A1: Concavity and convexity of $\mathrm{G}_{M}$ $\varepsilon \tau \sigma^{2} G_{M}$ (resp. 2/ $T_{M}{ }^{1-\sigma}$ ) is represented by the plain (resp. the dashed) curve Left panel: $\sigma=3, \tau=1, \varepsilon=1$; right panel $\sigma=6, \tau=2, \varepsilon=1$ 